\title{
Precision Muon Physics
}

\author{
T. P. Gorringe ${ }^{a}$, D. W. Hertzog ${ }^{b}$ \\ ${ }^{a}$ University of Kentucky, Lexington, $K Y$ 40506, USA \\ ${ }^{b}$ University of Washington, Seattle, WA 98195, USA
}

\begin{abstract}
The muon is playing a unique role in sub-atomic physics. Studies of muon decay both determine the overall strength and establish the chiral structure of weak interactions, as well as setting extraordinary limits on charged-leptonflavor-violating processes. Measurements of the muon's anomalous magnetic moment offer singular sensitivity to the completeness of the standard model and the predictions of many speculative theories. Spectroscopy of muonium and muonic atoms gives unmatched determinations of fundamental quantities including the magnetic moment ratio $\mu_{\mu} / \mu_{p}$, lepton mass ratio $m_{\mu} / m_{e}$, and proton charge radius $r_{p}$. Also, muon capture experiments are exploring elusive features of weak interactions involving nucleons and nuclei.

We will review the experimental landscape of contemporary high-precision and high-sensitivity experiments with muons. One focus is the novel methods and ingenious techniques that achieve such precision and sensitivity in recent, present, and planned experiments. Another focus is the uncommonly broad and topical range of questions in atomic, nuclear and particle physics that such experiments explore.
\end{abstract}

\section{Contents}

1 Introduction 3

1.1 Historical overview of muon experiments in sub-atomic physics . 3

1.2 Common features of precision muon experiments $\ldots \ldots \ldots 6$

$\begin{array}{lll}2 & \text { Muon lifetime } & 8\end{array}$

2.1 Fermi constant $G_{F}$. . . . . . . . . . . . . . . . . . . . . . 8

2.2 Experimental approaches to measuring $\tau_{\mu} \ldots \ldots$. . . . . . . . . . 9

2.2.1 MuLan experiment ............... . . . 10

$2.2 .2 \quad$ FAST experiment . . . . . . . . . . . . . . . . . . . . 12

2.3 Negative muon lifetime. . . . . . . . . . . . . . . . . . . 14

2.4 Results for the muon lifetime . . . . . . . . . . . . . . . . . . . 15

2.5 Results for the Fermi constant . . . . . . . . . . . . . . . 16

Preprint submitted to Progress in Nuclear and Particle Physics 
\begin{tabular}{lll}
\hline 3 & Muon decay & 17
\end{tabular}

$3.1 V-A$ structure of the weak interaction $\ldots \ldots \ldots \ldots . . \ldots 17$

3.2 TWIST experiment . . . . . . . . . . . . . . . . . . 20

3.3 Measurement of the positron longitudinal polarization . . . . . . 22

3.4 Measurement of the positron transverse polarization . . . . . . 2 25

3.5 Global analysis of decay parameters and theoretical implications 27

\begin{tabular}{lll}
\hline & Charged Lepton Flavor Violating decays & 30
\end{tabular}

4.1 Lepton flavor and physics beyond the standard model . . . . . . 30

4.2 Experimental challenge of muon cLFV . . . . . . . . . . . . . 34

$4.2 .1 \quad$ Measuring $\mu^{+} \rightarrow e^{+} \gamma \mid \ldots \ldots \ldots \ldots$. . . . . . . 35

$4.2 .2 \quad$ Measuring $\mu^{+} \rightarrow e^{+} e^{-} e^{+} \ldots \ldots \ldots \ldots \ldots$. . . . . 36

$4.2 .3 \quad$ Measuring $\mu^{-} N \rightarrow e^{-} N$ coherent conversion $\ldots \ldots$. . . 38

5 Muon Dipole Moments $\quad 40$

5.1 Terminology for muon magnetic and electric dipole moments . . 40

$5.2 \quad$ Magnetic dipole moment . . . . . . . . . . . . . . . . . 41

$5.2 .1 \quad$ New physics possibilities from $(g-2) \ldots \ldots$. . . . 44

5.3 Experimental considerations . . . . . . . . . . . . . . . 45

$5.3 .1 \quad$ Fermilab muon $(g-2)$ experiment . . . . . . . . . . 48

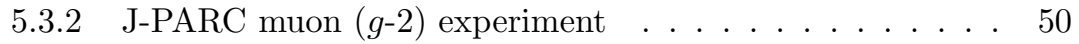

$5.3 .3 \quad$ Comparison of methods $\ldots \ldots \ldots \ldots$

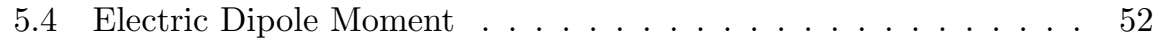

$5.4 .1 \quad$ Experimental Considerations $\ldots \ldots \ldots \ldots$

$\begin{array}{lll}6 & \text { Muonium hyperfine structure } & 57\end{array}$

6.1 Experimental approaches to muonium spectroscopy . . . . . . . . 57

6.2 LAMPF hyperfine structure experiment $\ldots \ldots \ldots \ldots$

6.3 MuSEUM hyperfine structure experiment . . . . . . . . . . . . 62

7 Muonic Lamb shift $\quad 63$

$\begin{array}{ll}7.1 & \text { Proton radius from electron scattering and hydrogen spectroscopy } 65\end{array}$

7.2 Muonic Lamb shift experiment . . . . . . . . . . . . . . 65

7.3 Present and future work involving muons . . . . . . . . . 67

$\begin{array}{lll}8 & \text { Nuclear muon capture } & 68\end{array}$

8.1 Basic features of muon capture . . . . . . . . . . . . 68

8.2 Muon capture on hydrogen, $\mu p \rightarrow n \nu$. . . . . . . . . . 69

8.2.1 Muon chemistry in pure hydrogen . . . . . . . . . . . . 71

$8.2 .2 \quad$ Experimental approaches to $\mu p$ capture . . . . . . . . . 72

$8.2 .3 \quad$ MuCap experiment . . . . . . . . . . . . . . . . . 73

8.3 Muon capture on deuterium, $\mu d \rightarrow n n \nu$. . . . . . . . . 77

8.3.1 Muon chemistry in pure deuterium . . . . . . . . . . 78

$8.3 .2 \quad$ MuSun experiment . . . . . . . . . . . . . . . . 80 
$9 \begin{array}{ll}\mathbf{S} & \mathbf{8 2}\end{array}$

9.1 Recent accomplishments . . . . . . . . . . . . . . . . . 83

9.2 Near-term projects . . . . . . . . . . . . . . . . . . 84

\section{Introduction}

The muon is not a building block of ordinary matter. It's much heavier than the electron but much lighter than the proton. It interacts through its electric charge and magnetic moment and its weak charged and neutral currents-but 5 not the strong force. Positive muons can form hydrogen-like atoms with electrons while negative muons can form hydrogen-like atoms with nuclei. The muon is unstable, but sufficiently long-lived to precisely study its properties and sufficiently short-lived to precisely study its decays. By a quirk of natureparity non-conservation-muons are produced fully polarized and when they decay they are self-analyzing.

Since its discovery the muon has played a rather unique and versatile role in physics. In this review we discuss recent, current and near-future efforts involving precision measurements of properties and decays of free muons and muonic atoms. The physics topics - which range from fundamental constants and basic

15 symmetries, to weak nucleonic and nuclear interactions, and standard model tests and new physics searches - are quite diverse. These unique experiments are generally designed to do one thing and do it well. We aim to provide an experimentalist's perspective into how these measurements are performed and their physics impact.

This article is organized as follows. Section 1.1 introduces the historical evolution of muon experiments in sub-atomic physics. Some general comments on muon facilities, experimental technologies and blind-analysis procedures are made in Sec. 1.2. Section 2 discusses the measurement of the muon lifetime and determination of the Fermi Constant. The measurements of the muon decay 25 parameters and tests of the $V$ - $A$ theory are described in Sec. 3 . Searches for charged lepton flavor violating muon decays are described in Sec. 4 . Section 5 covers the measurement of the muon's magnetic and electric dipole moments, and their sensitivities to new particles and unknown forces. Lastly, a number of precision measurements involving muonium $\left(\mu^{+} e\right)$ atoms and muonic $\left(\mu^{-} Z\right)$

30 atoms, which determine fundamental constants and elementary interactions, are discussed in Secs. 6, 7 and 8 .

\subsection{Historical overview of muon experiments in sub-atomic physics}

Table 1 summarizes the important properties and decay modes of the muon. The discovery of muons - new particles about 200 times more massive than 35 electrons - as cosmic-ray constituents was famously made by Anderson and Neddermeyer [1] at Caltech in 1936 and Street and Stevenson [2] at Harvard in 1937. Their work was actually the culmination of numerous experiments conducted over many years, which eventually demonstrated that the highly-penetrating 
Table 1: Summary of Measured Muon Properties and Selected Decay Rates and Limits

\begin{tabular}{lcccc}
\hline Property & Symbol & Value & Precision & Ref. \\
\hline Mass & $m_{\mu}$ & $105.6583715(35) \mathrm{MeV}$ & $34 \mathrm{ppb}$ & {$[4]$} \\
Mean Lifetime & $\tau_{\mu}$ & $2.1969811(22) \times 10^{-6} \mathrm{~s}$ & $1.0 \mathrm{ppm}$ & {$[5]$} \\
Anom. Mag. Moment & $a_{\mu}$ & $116592091(63) \times 10^{-11}$ & $0.54 \mathrm{ppm}$ & {$[4]$} \\
Elec. Dipole Moment & $d_{\mu}$ & $<1.9 \times 10^{-19} e \cdot \mathrm{cm}$ & $95 \%$ C.L. & {$[7]$} \\
\hline \hline Branching Ratios & PDG average & B.R. Limits & $90 \%$ C.L. & Ref. \\
\hline$\mu^{-} \rightarrow e^{-} \bar{\nu}_{e} \nu_{\mu}$ & $\approx 100 \%$ & $\mu^{-} \rightarrow e^{-} \gamma$ & $5.7 \times 10^{-13}$ & {$[8]$} \\
$\mu^{-} \rightarrow e^{-} \bar{\nu}_{e} \nu_{\mu} \gamma$ & $1.4(4) \%$ & $\mu^{-} \rightarrow e^{-} e^{+} e^{-}$ & $1.0 \times 10^{-12}$ & {$[9]$} \\
$\mu^{-} \rightarrow e^{-} \bar{\nu}_{e} \nu_{\mu} e^{+} e^{-}$ & $3.4(4) \times 10^{-5}$ & $\mu^{-} \rightarrow e^{-}$conversion & $7 \times 10^{-13}$ & {$[10]$} \\
\hline
\end{tabular}

component of cosmic radiation was neither electrons nor protons 11 Ultimately,

40 the discovery became our first evidence for generations of elementary particles and the hierarchical structure of the standard model.

The first cloud chamber photograph of the decay of a muon was taken in 1940 [11. The earliest determination of the muon lifetime was made by interpreting the "anomalous absorption" of cosmic-ray muons with decreasing alti45 tude as muon disintegration [12. The direct measurement of the muon lifetime was made shortly afterwards by recording the time intervals between stopping muons and decay electrons using cosmic rays 13 . These early measurementsinvolving in-flight and stopped muons - afforded a decisive test of time dilation for moving particles. Nowadays the precision measurement of the muon life${ }_{50}$ time $\tau_{\mu}$ provides the best determination of the Fermi constant $G_{F}$, the quantity governing the universal strength of weak interactions.

The $\mu \rightarrow e \nu \bar{\nu}$ decay scheme was established through cosmic-ray measurements of the decay electron energy spectrum. It was demonstrated-via the continuum distribution and the energy endpoint - that muons decay into three

55 or more particles with small or zero masses 14. Fermi's theory of nuclear $\beta$-decay was consequently expanded to accommodate $\mu \rightarrow e \nu \bar{\nu}$ decay thus affording the first glimpse of weak universality. These early experiments - along with theoretical work on the tensor structure of the current-current interaction [15, 16] - were the beginnings of our modern precision studies of the muon decay parameters as a valued probe of the weak force.

In a profound paper in 1956, Lee and Yang suggested that the discrete symmetry of parity might be violated in the weak interaction [17]. One important prediction of parity non-conservation concerned the by-then well-known $\pi \rightarrow \mu \rightarrow e$ weak decay chain. First, the non-conservation of parity in $\pi \rightarrow \mu \bar{\nu}$

65 decay would cause muons to be polarized along the muon momentum axis. Second, the non-conservation of parity in $\mu \rightarrow e \nu \bar{\nu}$ decay would cause electrons to be emitted anisotropically about the muon polarization axis. Soon afterwards

\footnotetext{
${ }^{1}$ The existence of highly-penetrating cosmic rays was known since the work of Bothe and Kolhorster in 1929. In 1933, Kunze [3] noted a "particle of uncertain nature" in his investigations of cosmic rays using ionization chambers.
} 
the non-conservation of parity in $\pi \rightarrow \mu \rightarrow e$ decay was reported by Garwin et al. [18, and Friedman and Telegdi [19], following their observation of large 70 decay-electron anisotropies. Parity non-conservation is now codified in the $V$ - $A$ structure of the weak currents 20, 21, which imparts a distinctive angle-energy correlation on the decay electrons with the muon polarization. Since this early work, the precision measurement of angle-energy correlations has enabled increasingly precise tests of $V$-A theory.

The hypothesis of a weak interaction mediated by a force-carrying boson was originally introduced in 1940 [22]. The early theories involving weak bosons predicted the existence of $\mu \rightarrow e \gamma$ decay at the level of about $10^{-4}$ (the mechanism for $\mu \rightarrow e \gamma$ involved the neutrino emitted by the muon being absorbed by the electron). By the late 1950s the $\mu \rightarrow e \gamma$ experimental limit was orders-ofso magnitude below this theoretical prediction. The crisis concerning $\mu \rightarrow e \gamma$ decay led Pontecorvo 23 and others to postulate the existence of two distinct neutrino types; an electron-flavored neutrino and a muon-flavored neutrino. Since the genesis of lepton flavor in $\mu \rightarrow e \gamma$, increasingly delicate searches for rare processes including $\mu \rightarrow e \gamma$ and $\mu \rightarrow e$ conversion have continued to shape our 5 understanding of flavor.

The aforementioned Garwin et al. experiment on parity non-conservation in muon decay also yielded the first measurement of the magnetic moment of the positive muon. Their result for the gyromagnetic ratio $g=+2.00 \pm$ 0.10 was a demonstration that the muon was a structureless, spin- $1 / 2$ Dirac

90 particle. Subsequently - through increasingly sophisticated measurements that utilize the possibilities of polarization and polarimetry of muons in $\pi \rightarrow \mu \rightarrow e$ decay - the determination of the anomalous part $(g-2)$ of the muon magnetic moment has been measured to an astonishing sub-part-per-million level. The anomalous moment arises through quantum vacuum fluctuations that accrue 95 from all particles of nature - both known and unknown - and thereby affords a unique window on new physics at high-energy scales

The simplest atom involving muons is muonium, a pure QED bound state of $\mu^{+} e^{-}$, sometimes dubbed the "perfect atom." Because both constituents are point-like leptons the muonium energy levels are completely free of perturbations 100 arising from nuclear size effects. Its formation was first identified by detecting the characteristic Larmor precession frequency of polarized muonium formed when muons stop in certain gases. Following its discovery the measurement of the hyperfine splitting of the muonium ground state - by inducing microwave transitions between hyperfine states in external magnetic fields - was developed by Hughes and co-workers (for details see 24]). This work has provided the most precise determinations of the muon-to-proton magnetic moment ratio $\mu_{\mu} / \mu_{p}$ and the muon-to-electron mass ratio $m_{\mu} / m_{e}$ as well as important tests of quantum electrodynamics.

When negative muons are brought to rest in matter they undergo atomic cap110 ture and form muonic atoms. These atoms are hydrogen-like systems whereunimpeded by the exclusion principle - the muon cascades from an initially high principal quantum number state to the $1 S$ atomic ground state. The existence of such atoms was first discussed in Refs. [25, 26] that demonstrated the timescale 
for formation of muonic atoms was much shorter than the muon lifetime. Compared to ordinary atoms, the muonic atom radii are $\left(m_{\mu} / m_{e}\right)$ times smaller and the energy levels are $\left(m_{\mu} / m_{e}\right)$ times greater. Consequently, the overlap between the muon orbits and the nucleus is much larger than in ordinary atoms and the energy levels can be significantly perturbed by the nuclear charge distribution. Precision spectroscopy of muonic atoms thus became a workhorse for studies of 120 nuclear charge radii and electromagnetic moments. In the simplest case of a $\mu p$ atom, the recent measurements of the tiny energy splitting (Lamb shift) between the muonic $2 S_{1 / 2}-2 P_{1 / 2}$ orbitals have provided the most precise determination of the proton charge radius.

After muonic atoms are formed they disintegrate by either muon decay or nuclear capture $\mu^{-}[A, Z] \rightarrow[A, Z-1] \nu$. The first experimental evidence for muon capture was observations of differing electron yields when stopping negative and positive muons in carbon and iron [27. This work demonstrated the weak nature of muon capture on atomic nuclei thus dispelling the notion that cosmic-ray muons were the force-carriers of the strong interaction, and ultimately leading to early ideas of weak universality in muon capture, muon decay and beta decay. Modern measurements of muon capture are investigating weak nucleonic and nuclear interactions that address subjects which range from standard model symmetries to fundamental astrophysical processes.

\subsection{Common features of precision muon experiments}

Muon beams are derived from pion decays, the pions being produced in the nuclear collisions between an accelerated beam and a fixed target. There are three basic types of muon beam lines involving so-called surface, cloud and decay muons. In surface $\mu^{+}$beams the muons originate from at-rest decay of $\pi^{+}$stops in the surface layer of the production target. The resulting muons are mono-energetic $29.8 \mathrm{MeV} / \mathrm{c}, 100 \%$ longitudinally polarized, and because of the localized source have a sharp focus ${ }^{2}$ In cloud beams the muons originate from in-flight decays of parent pions in the region between production target and the first bending magnet of the secondary beam line. The resulting muons arise from "forward-decays" and "backward-decays" of pions and therefore the resulting polarization is considerably lower than surface beams. In decay beams an upstream section of the beam line selects the parent pion momentum and a downstream section of the beam line selects the daughter muon momentum. The resulting muons are typically highly polarized and free of electron contamination.

Intense muon sources at the Paul Scherrer Institute (PSI) in Switzerland and TRIUMF in Canada are examples of muon beams based on high current, medium energy, proton cyclotrons that provide an essentially continuous beam (with the micro-time structure of the cyclotron radio-frequency). Facilities at Fermilab in the U.S. and J-PARC in Japan are examples of muon beams based

\footnotetext{
${ }^{2}$ Only surface $\mu^{+}$beams are available as $\pi^{-}$stops form pionic atoms and rapidly undergo nuclear capture.
} 
on lower current, higher energy, proton synchrotrons and provide a pulsed beam with typical repetition rates of tens of Hertz. Additionally, novel muon sources for ultra-cold muons, based on formation and ionization of muonium atoms, and ultra-intense muons, based on capture and transport by superconducting solenoids, are under development for future muon experiments (see Secs. 5.3 .2 and 4.2.3 respectively).

Today's precision muon experiments - which require both enormous statistics and extraordinary limits on possible biases from systematic effects - are benefiting from advances in areas including radiation detectors, readout electronics, computer hardware and software infrastructure.

Challenges for detector design include stringent requirements on timing, tracking and calorimetry in high-rate environments. Ultra-low mass tracking chambers and modern silicon detector technology have been used or are being developed for high-rate, high-precision tracking applications. High-density electromagnetic calorimeters (liquid xenon, lead fluoride, lead tungstate) have been built or are being constructed for both good energy resolution and fast-timing applications. Modern silicon photomultipliers are enabling readout of scintillation and Cherenkov light in magnetic fields and restricted geometries. And sophisticated NMR-based field measurements and finite-element field modeling are being utilized for precision magnetometry.

Commercial applications for real-time measurement, processing and distribution of massive data-streams have driven hardware development including high sampling-rate waveform digitizers, field programmable gate arrays and graphical processing units. The digital capture of detector signals offers invaluable opportunities for systematics investigations in precision measurements and waveform digitizers have been used or are being deployed in many recent, present and future experiments. Field programmable gate arrays-i.e. highperformance user-customizable integrated circuits - are now common for special purpose tasks in timing, trigger and control logic. A hybrid architecture of multicore CPUs and teraflop-performance GPUs is being developed for real-time readout and processing in the Fermilab muon $(g-2)$ experiment.

Recent precision muon experiments have stored datasets of hundreds of terabytes of raw data and future experiments will store datasets of many petabytes of raw data. This scale of data analysis and data simulations has required grid computing facilities such as WesGrid (Canada) and the National Center for Supercomputing Applications (U.S.). The extreme demands on high statistics and understanding systematics are pushing applications of GEANT (GEometry ANd Tracking software toolkit) into new territories.

Increasingly, modern measurements are carried out using so-called "blindanalysis" techniques. A simple example for measurements that use precision oscillators for timing is to prescriptively de-tune the oscillator by a small offset from its nominal setting during the data taking; the offset is unknown to anyone analyzing the data. The data analysis time unit is then a somewhat arbitrarily defined "clock tick." When the analyses are complete, the clock ticks are converted into physical time units, and the unblinded result is revealed. In efforts where rare events are investigated, blinded regions must be established 
a)

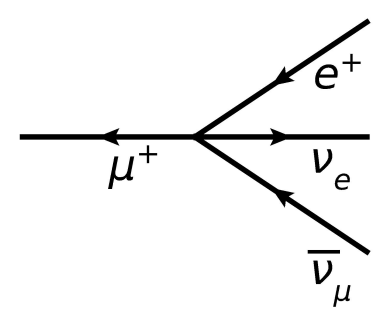

b)

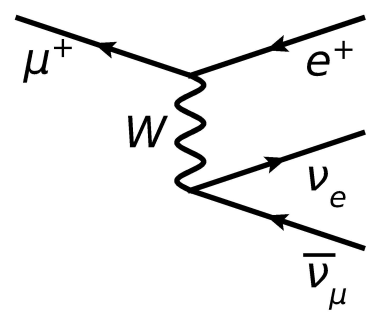

Figure 1: a). Tree-level Feynman diagram for ordinary muon decay in Fermi's current-current interaction. b). Tree-level standard model diagram for ordinary muon decay indicating the W-boson mediated weak interaction between the leptonic currents.

in which the rare events would be found. Backgrounds and cuts are determined from allowed events outside and near the forbidden box. Once the analysis is complete, the blinded box is opened to see if any events have survived. These disciplined procedures provide a needed level of integrity to the experiments.

\subsection{Fermi constant $G_{F}$}

The strength of the weak interaction is governed by the Fermi constant $G_{F}$. The roots of $G_{F}$ are Fermi's theory-based on an analogy between the emission of an electron-neutrino pair by a radioactive nucleus and a photon 1934, our modern understanding of weak interactions has evolved to incorporate parity-violating $V-A$ currents and the massive $W$ and $Z$ gauge bosons. However, the constant $G_{F}$ and Fermi interaction have survived as a convenient, low energy, effective theory of the weak sector in the standard model (and presumably any successor).

Within the standard model the Fermi constant (see Fig. 1) is given by

$$
\frac{G_{F}}{\sqrt{2}}=\frac{g^{2}}{8 M_{W}^{2}}\left(1+\sum_{i} r_{i}\right)
$$

where $1 / M_{W}^{2}$ represents the tree-level propagator corresponding to $W$-boson exchange and $g$ the weak coupling. The term $\sum_{i} r_{i}$ incorporates the higherorder electroweak interaction corrections [28. The factors of $\sqrt{2}$ and 8 in Eqn. 1 are reminders of the origins of the Fermi constant in a vector current - vector 220 current weak interaction.

By far the best determination of the Fermi constant is obtained by the measurement of the positive muon lifetime, $\tau_{\mu}$. Experimentally, intense beams of low-energy muons are nowadays available and the $2.2 \mu$ s muon lifetime with its associated decay electrons are nicely suited to precision measurements of 

and $\mu \rightarrow e e e \nu \bar{\nu}$-are pure leptonic weak interactions, their interpretation is unambiguous.

The determination of the Fermi constant $G_{F}$ from the muon lifetime $\tau_{\mu}$ represents a reference point for subatomic physics. It permits the testing of 230 weak universality, for example, through precision measurements of leptonic taudecays. It enables the determination of weak mass-mixing angles, for example, through precision measurements of neutron decay. Moreover - together with the fine structure constant $\alpha$ and the $\mathrm{Z}$ gauge boson mass $M_{Z}$-it completely determines the electroweak sector of the standard model and enables searches

\subsection{Experimental approaches to measuring $\tau_{\mu}$}

As already discussed, after the discovery of the muon by Anderson and Neddermeyer [1] and Street and Stevenson 2] the earliest measurements of the lifetime - for stopped muons and in-flight muons - were important in verifying the time dilation of moving particles.

By the beginning of this century the Particle Data Group world average of the muon lifetime was $\tau_{\mu}=2.19703 \pm 0.00004 \mu$ s or 18 parts-per-million (ppm) 29]. The world average was largely determined by three measurements: Giovanetti et al. 30, Bardin et al. 31] and Balandin et al. 32, that were conducted in the seventies and the eighties. The experiments of Giovanetti et al. and Balandin et al. used low-rate continuous beams in order to insure the arrival and decay of muons occur one-by-one, i.e. avoiding any incorrect assignment of daughter electrons with parent muons. At higher rates, if only the previous stop was associated with a particular electron the measured lifetime would be 250 distorted, or, if all the neighboring stops were associated with a particular electron a random background would be incurred. Such one-by-one measurements therefore limit the collection of decays to roughly $10^{10}$ and the uncertainty on $\tau_{\mu}$ to roughly $10 \mathrm{ppm}$.

The two most recent measurements of the positive muon lifetime - the FAST experiment 33 and MuLan experiment 5] - were specifically designed to circumvent the statistical limitations of one-by-one measurements. The FAST approach involved an active pixelated target in order to reconstruct muonelectron vertices and thereby correctly associate each decay electron with its parent muon. In principle therefore, multiple muons could be simultaneously 260 stopped and multiple decays could be simultaneously recorded, without losing the parent-daughter association. The MuLan approach involved a timestructured muon beam in order to first prepare a "radioactive source" of muons and afterwards measure the "emanating radiation" of electrons. In this scheme no association of a particular daughter electron with a particular parent muon 265 is necessary; the observed lifetime of a radioactive source is independent of the source preparation. 


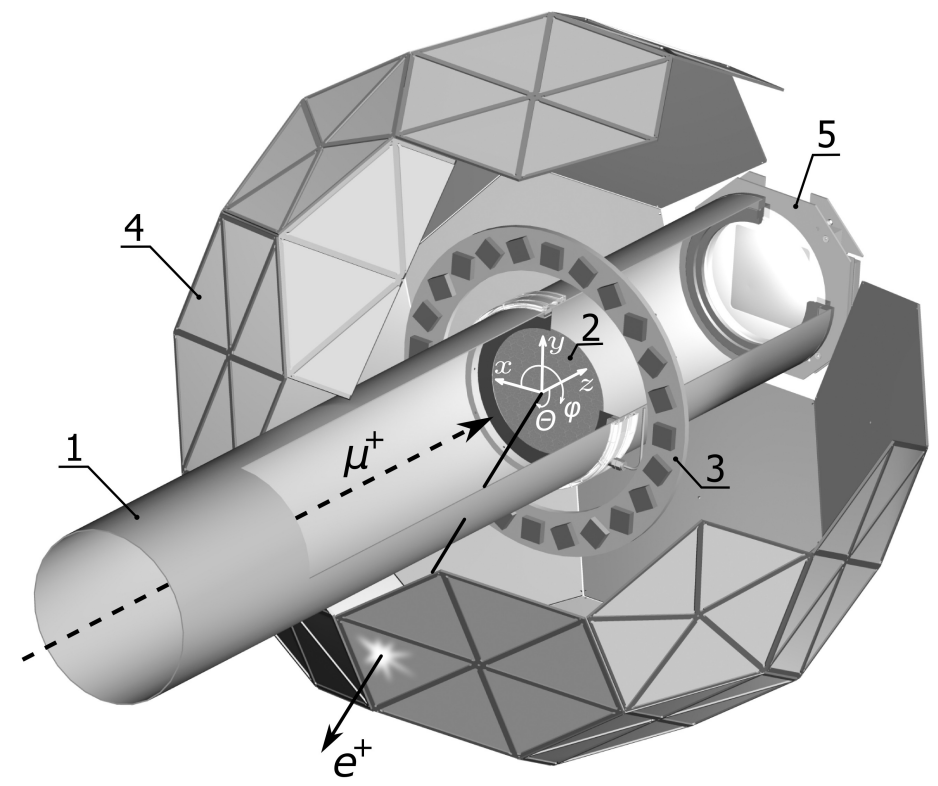

Figure 2: Cutaway diagram of the MuLan experiment indicating an incoming muon and outgoing positron and showing the beam pipe (1), stopping target (2), optional magnet array (3), scintillator detector (4) and beam monitor (5). Figure courtesy MuLan collaboration.

\subsubsection{MuLan experiment}

The MuLan experiment [5] was conducted at PSI. The setup-which comprised an in-vacuum stopping target and fast-timing finely-segmented positron detector - is shown in Fig. 2 2 The setup was designed to both accumulate the necessary quantity of muon decays and minimize distortions arising from muon spin rotation in the stopping target and electron pile-up in the detector array.

To reach the necessary statistics of $10^{12}$ decays the experiment relied on a time-structured surface-muon beam (see Fig. 3). The experiment involved cycles of $5 \mu$ s duration beam-on periods to accumulate stopped muons and $22 \mu$ s duration beam-off periods to measure decay positrons. The arrangement permitted an average rate of stopped muons of $1-2 \times 10^{6} \mathrm{~s}^{-1}$; much more than permissible in a one-by-one lifetime measurement.

Surface muon beams are nearly $100 \%$ longitudinally polarized. The positrons emitted in muon decay are distributed asymmetrically about the $\mu$-spin axis with high-energy positrons preferentially emitted in the spin direction and low energy positrons preferentially emitted opposite the spin direction. The spin vectors of stopped muons both precess and relax in the local magnetic field of the target material; a phenomenon known as muon spin rotation. $\mu \mathrm{SR}$ yields a timedependent muon-ensemble polarization and thereby a time-dependent decaypositron angular distribution. It results - when detecting positrons in specific directions - in a geometry-dependent modulation of the exponential decay curve 


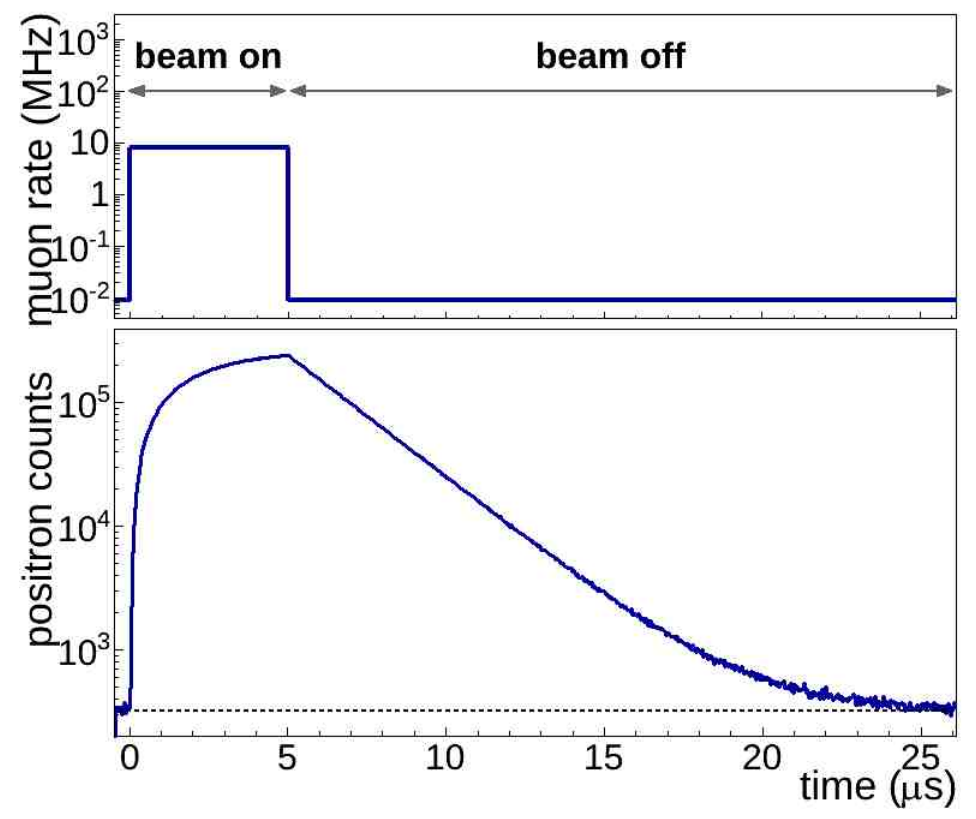

Figure 3: The 5- $\mu$ s beam-on, $22-\mu$ s beam-off, time structure of the MuLan experiment. The upper panel shows the muon arrival time distribution and the lower panel shows the decay electron time distribution.

by the $\mu$ SR signal.

Two combinations of stopping target material and environmental magnetic fields were used. One configuration involved a magnetized ferromagnetic foil with a roughly $0.5 \mathrm{~T}$ internal field orientated perpendicular to the beam axis. Another configuration involved a nonmagnetic quartz crystal with a roughly $80 \mathrm{G}$ external field orientated perpendicular to the beam axis. Muon stops in the ferromagnetic target were mostly diamagnetic $\mu^{+}$ions exhibiting a precession frequency of $13.6 \mathrm{kHz}$ per Gauss while muon stops in the quartz target were mostly paramagnetic $\mu^{+} e^{-}$atoms exhibiting a precession frequency of $1.39 \mathrm{MHz}$ per Gauss. The targets were mounted in the beam vacuum to avoid either multiple scattering or stopping in upstream detectors or vacuum windows.

The $5 \mu$ s duration of muon accumulation was important in dephasing the spins of the polarized stopping muons in the transverse magnetic fields of the two target configurations. In both cases the precession frequencies of either $\mu^{+}$ions or $\mu^{+} e^{-}$atoms was sufficient to reduce the transverse polarization by roughly a thousand-fold. The residual polarization was ultimately limited by the alignment accuracy between the transverse field and the beam polarization, i.e. the presence of a small longitudinal component of the muon polarization.

The positron detector comprised a nearly $4 \pi$ array of 170 fast-timing, doublelayered, scintillator detectors arranged in a truncated icosahedron (soccer ball) geometry. The high granularity and fast timing characteristics were important in 
minimizing the incidence of positron pile-up. The forward-backward symmetry of the $3 \pi$ solid angle detector also suppressed the imprint of $\mu \mathrm{SR}$ on the time distribution of the decay positrons ${ }^{3}$ Therefore the MuLan $\mu$ SR signal is strongly suppressed and largely determined by the the non-uniformity of the detection efficiency.

Analog signals from detector elements were digitized using fast-sampling 315 ADCs and digitized "islands" of contiguous samples of above-threshold signals were identified by FPGAs. A distributed data acquisition enabled the storage of all above-threshold signals from the positron detector.

A temperature-stabilized crystal oscillator was used as the timebase for the fast-sampling ADCs. The collaboration was blinded to the exact frequency of the timebase during the data taking and the subsequent analysis. Only after completing the entire analysis was the frequency unblinded and the lifetime revealed.

In analyzing the data the digitized islands were first fit to pulse templates to determine the times and energies of individual pulses. A software-defined min325 imum amplitude was applied to distinguish the minimally ionizing positrons from low-energy backgrounds and a software-defined minimum deadtime was applied to establish an explicit resolving time between neighboring pulses. The time distribution of coincident hits between inner-outer tile pairs was then constructed.

After the application of small data-driven corrections for positron pileup and gain changes, the time distributions of coincident hits were fit to extract $\tau_{\mu}$. The ferromagnetic target data showed no evidence of $\mu \mathrm{SR}$ effects and was fit to the function

$$
N(t)=N e^{-t / \tau_{\mu}}+B
$$

330 where the time-independent background $B$ arose from both cosmic rays and the imperfect beam extinction during the measurement period. The quartz target data showed a clear $\mu \mathrm{SR}$ signal and was therefore fit to a modified function that incorporated both longitudinal- and transverse-field $\mu \mathrm{SR}$ effects. The fits were performed for different software deadtime and extrapolated to zero software 335 deadtime to obtain $\tau_{\mu}$.

The values of $\tau_{\mu}$ obtained from the two targets were in good agreement and yielded a combined result of

$$
\tau_{\mu}=2196980.3 \pm 2.1 \text { (stat) } \pm 0.7 \text { (syst) ps; }
$$

an overall uncertainty of $2.2 \mathrm{ps}(1.0 \mathrm{ppm})$ and thirty-fold improvement over earlier generations of experiments.

\subsubsection{FAST experiment}

The FAST experiment 33 was also conducted at PSI. The setup-which consisted of beam defining counters and a pixelated stopping target-is shown

\footnotetext{
${ }^{3}$ A detector with perfectly forward-backward symmetry about the muon spin direction would display no $\mu \mathrm{SR}$ signal in the positron time distribution.
} 


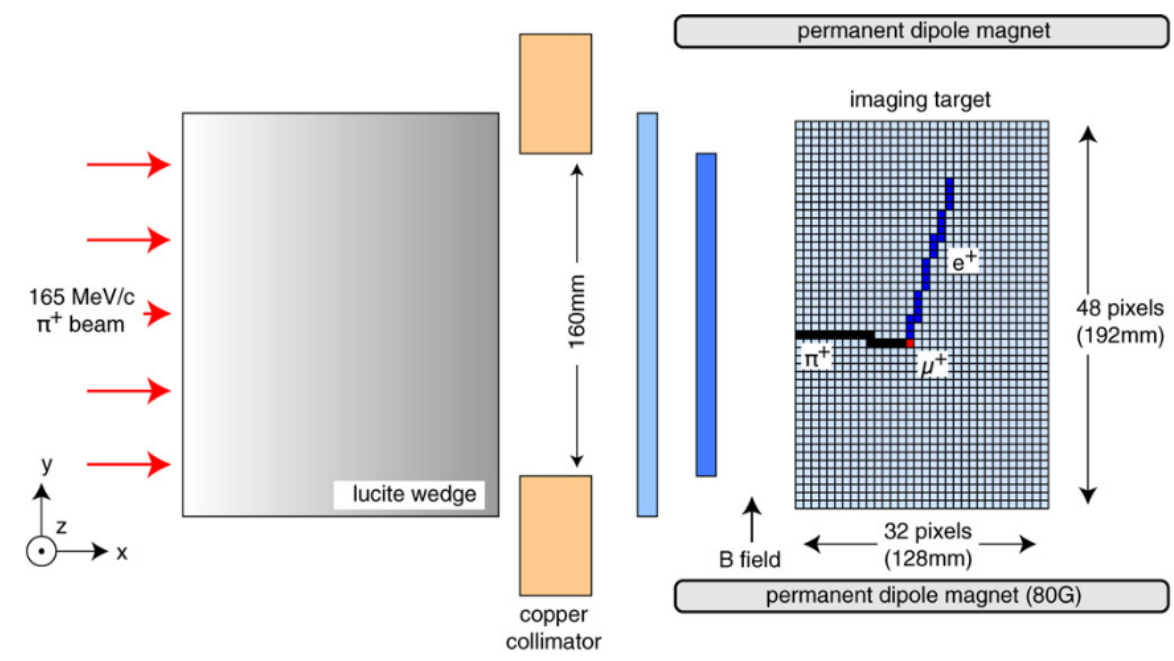

Figure 4: Horizontal cross section of the FAST experiment showing (from left to right) the wedge-shaped degrader, beam defining scintillators and pixelated stopping target. The pattern of hits from a $\pi \rightarrow \mu \rightarrow e$ decay chain are also indicated. Figure courtesy FAST collaboration.

in Fig 4

The approaches of FAST and MuLan to measuring $\tau_{\mu}$ were very different. In FAST the segmented target was used to associate the daughter positrons with parent muons by identifying the coordinates of the muon stop and the trajectory 345 of the decay electron.

Additionally, the FAST experiment employed a stopping pion beam. When pions were stopped the subsequent muons from $\pi \rightarrow \mu \nu$ decay and subsequent positrons from $\mu \rightarrow e \nu \bar{\nu}$ decay were detected, thereby identifying the entire $\pi \rightarrow \mu \rightarrow e$ decay chain. Since the decay muons from stopped pions are emitted isotropically, the overall ensemble of stopped muons had essentially zero polarization. This minimizes the $\mu \mathrm{SR}$ distortions of the decay-positron time distribution.

To widely distribute the stopping pions over the segmented target the beam was defocused at the target location and directed through a wedge-shaped de-

355 grader. The degrader varied the penetration depth of pions according to their vertical coordinate. Beam counters were situated both upstream and downstream and provided the identification of stopping pions and through going electrons. A permanent magnet comprising two planes of ferrite blocks provided a $80 \mathrm{G}$ transverse magnetic field.

360 The segmented stopping target comprised a $32 \times 48$ pixelated array of $4 \times$ $4 \times 200 \mathrm{~mm}^{3}$, vertically orientated, plastic scintillator bars. The light from individual bars was readout by multi-anode photomultipliers. After amplification and discrimination the hit times of individual pixels were recorded by multihit TDCs. The setup employed two discriminator thresholds, a higher-level thresh- 

the trigger logic and a lower-level threshold capable of identifying the minimally ionizing positrons that was used in the data analysis.

In normal data-taking the TDC information was readout after the identification of a pion stop and a corresponding decay muon in the pixelated target.

370 A level-one trigger distinguished the incident pions from other beam particles. A level-two trigger identified the sequence involving the prompt pion signal and the delayed muon signal (it used an FPGA array to perform the trigger decision within several microseconds). The range of the $4.1 \mathrm{MeV}$ muons from the $\pi \rightarrow \mu \nu$ decay was approximately $1.5 \mathrm{~mm}$ and therefore the muon stop was located in either the same pixel or an adjacent pixel to the pion stop. On fulfilling the above trigger the pion stop pixel was used to define a $7 \times 7$ pixel region and a -8 to $+22 \mu$ s time window for the selective readout of the TDC modules.

Data were collected at incident beam rates of roughly $160 \mathrm{kHz}$ and stopping pion rates of roughly $80 \mathrm{kHz}$, and yielded a sample of approximately $1.0 \times 10^{10}$ was both readout and also processed in real-time. The real-time processing used lookup tables to identify the characteristic topologies of pixel hits corresponding to a positron emanating from the muon stop (the topologies allowed for pixel inefficiencies).

385 The identification of $\pi \rightarrow \mu \rightarrow e$ events yielded the corresponding times $t_{\pi}, t_{\mu}$ and $t_{e}$ of the pion, muon and positron and thereby the time differences between the stopped pion and the decay electron, $t_{e}-t_{\pi}$, and between the stopped muon and the decay electron, $t_{e}-t_{\mu}$. This real-time processing accumulated both global $t_{e}-t_{\pi}$ and $t_{e}-t_{\mu}$ histograms for all $\pi \rightarrow \mu \rightarrow e$ events as well as sub-sets for different pion coordinates, positron topologies, etc. Both the measured $t_{e}-t_{\pi}$ and $t_{e}-t_{\mu}$ distributions exhibit the muon lifetime ${ }^{4}$

The time distributions showed the exponential decay curve as well as timeindependent and cyclotron-RF correlated backgrounds. The two backgrounds arose from random backgrounds and beam particles that generated fake positron 395 topologies and thereby $\pi \rightarrow \mu \rightarrow e$ events. The time distributions showed no evidence of $\mu \mathrm{SR}$ effects.

The RF-correlated background was handled by rebinning data with the cyclotron RF period. After the rebinning procedure a maximum likelihood fit to the decay curve gave the final result

$$
\tau_{\mu}=2197083 \pm 32 \text { (stat) } \pm 15 \text { (syst) ps }
$$

with an overall uncertainty of $35 \mathrm{ps}(16 \mathrm{ppm})$.

\subsection{Negative muon lifetime}

The above experiments involved measurements of the lifetime $\tau_{\mu}$ of the pos-

\footnotetext{
${ }^{4}$ The $t_{e}-t_{\pi}$ distribution is a single decay curve with lifetime $\tau_{\mu}$ for time intervals much greater than $\tau_{\pi}$.
} 
addition to muon decay the process of muon capture is possible. Measurement of the negative muon lifetime in a particular stopping material therefore determines a total disappearance rate, i.e. the sum of the decay rate and the capture rate. The contribution of the capture rate to the disappearance rate varies from roughly $0.1 \%$ in hydrogen isotopes to greater than $90 \%$ in heavy elements.

In principle the lifetime of free negative muons can be determined by the combined measurements of the disappearance rate and the capture rate for a specific stopping target. For muon stops in hydrogen and deuterium there exist both measurements of the muon disappearance rates $\left(\Lambda_{D}\right)$ via the decay electron time spectra and the total muon capture rates $\left(\Lambda_{C}\right)$ via the capture neutron yields. Thus the combination of these measurements allows the determination of the free negative muon lifetime using

$$
1 / \tau_{\mu^{-}}=\Lambda_{D}-\Lambda_{C} .
$$

Unfortunately, the procedure is complicated by muon atomic and molecular processes that occur in isotopes of hydrogen. Consequently, to directly extract the lifetime $\tau_{\mu^{-}}$the values of $\Lambda_{D}$ and $\Lambda_{C}$ must be determined for the same muonic atomic and molecular state populations. We note that together - the recent measurement of the muon disappearance rate [34] and the earlier measurements of the muon capture rate [35, 36] from the singlet $\mu^{-} p$ atom-yield using Eqn. 2 a negative lifetime that is entirely consistent with the positive muon lifetime 5

\subsection{Results for the muon lifetime}

The uncertainty of the MuLan measurement of the muon lifetime is about 30-40 times smaller than the previous generation of lifetime experiments and about ten times smaller than the published result from the FAST experiment. While the MuLan result is in reasonable agreement with the earlier experiments there is some tension between the MuLan experiment and the FAST experiment.

${ }_{420}$ The weighted average of all results gives a lifetime $\tau_{\mu}=2196981.1 \pm 2.2 \mathrm{ps}$ with a chi-squared value that is dominated by the $2 \sigma$ difference between MuLan and FAST.

As discussed in Sec. 8, the accurate knowledge of $\tau_{\mu}$ is important to precision measurements in muon capture. The MuCap experiment [37] has recently measured the $\mu^{-}$p singlet capture rate $\Lambda_{s}$ and the MuSun experiment 38 is currently measuring the $\mu^{-} \mathrm{d}$ doublet capture rate $\Lambda_{d}$. Both experiments derive these capture rates from the tiny difference $\left(\Lambda_{o}-\Lambda\right)$ between the positive muon decay rate $\left(\Lambda_{o}=1 / \tau_{\mu^{+}}\right)$and the muonic atom disappearance rates $\left(\Lambda=1 / \tau_{\mu Z}\right)$. Because the capture rates are very small, very precise determinations of both the muonic atom disappearance rates and the free muon decay rate are necessary.

\footnotetext{
${ }^{5}$ Small corrections for $\mu^{-} p$ bound state effects and $p p \mu$ molecular formation are required to extract of the negative muon decay rate from the measured values of the disappearance rate and the capture rate. See Ref. [34 for details.
} 
Prior to this work the muon decay rate $\Lambda_{o}$ was known to $9 \mathrm{ppm}$ or $5 \mathrm{~s}^{-1}$, an uncertainty that limited the capture rate determinations from disappearance rate experiments. The recent work on the muon lifetime - yielding a precision of $1 \mathrm{ppm}$ or $0.5 \mathrm{~s}^{-1}$ in $\Lambda_{o}$-has eliminated this source of uncertainty. sector of the standard model.

Within the standard model the quantities $\alpha, M_{Z}$ and $G_{F}$ are related to other fundamental quantities that include the charged weak boson mass $M_{W}$

${ }^{6}$ This value for $G_{F}$ is also listed in the 2014 compilation of the physical constants by the Particle Data Group [43. 
and weak mixing angle $\theta_{W}$. Such SM relationships have radiative corrections that impart sensitivities to the top quark mass $m_{t}$ and the Higgs boson mass $m_{h}$. Historically, precision electroweak data was important in constraining the then-unknown masses of the top quark and the Higgs boson.

The top quark was discovered in 1995 at Fermilab by the CDF and D0 experiments [44, 45] and the Higgs boson was discovered in 2012 at CERN by the ATLAS and CMS experiments [46, 47]. The spectacular agreement between the forecast and the measurement of top mass $m_{t}=173 \mathrm{GeV} / \mathrm{c}^{2}$ was a huge triumph for the predictive power of the standard model. Through the SM relations for the Higgs sector 48

$$
\begin{aligned}
G_{F} & =\frac{1}{\sqrt{2} v^{2}} \\
m_{h} & =2 \nu^{2} \lambda
\end{aligned}
$$

our knowledge of $G_{F}$ and $m_{h}$ are sufficient to determine the two parametersthe vacuum expectation value $v$ and self-interaction parameter $\lambda$ - of the SM Higgs potential.

The Fermi constant obtained from the muon lifetime is the anchor of the universality tests of the weak force. Comparison between the purely leptonic decays of the tau and the muon-i.e., $\tau \rightarrow \mu \nu \bar{\nu}, \tau \rightarrow e \nu \bar{\nu}$ and $\mu \rightarrow e \nu \bar{\nu}$-are natural opportunities for testing the universality of leptonic weak interactions across the three generations. Using the available data on the $\tau$ lifetime and its 475 purely leptonic $\mu \nu \bar{\nu}$ and $e \nu \bar{\nu}$ branching ratios the leptonic universality of weak interactions has been demonstrated to the levels of several parts-per-thousand [43.

The Fermi constant is also the metric for extracting the Cabibbo-KobayashiMaskawa (CKM) matrix elements which specify the flavor mixing in weak interactions of quarks. The concept of flavor mixing of quark states was originally introduced by Cabibbo to explain the different strengths of strangeness-changing and strangeness-conserving weak interactions and thereby save weak universality. Modern tests of weak universality for three quark generations are therefore based on testing whether the sum of all couplings of an up-type quark (up, charm or top) to all down-type quarks (down, strange and bottom) is equal to unity. For the case of the up-quark, one obtains 49]

$$
\left|V_{u d}\right|^{2}+\left|V_{u s}\right|^{2}+\left|V_{u b}\right|^{2}=0.99978(55)
$$

which demonstrates weak universality between the quark-lepton sectors at the level of about 0.6 parts-per-thousand.

\section{1. $V$-A structure of the weak interaction}

Herein we discuss the decay $\mu^{+} \rightarrow e^{+} \nu_{e} \overline{\nu_{\mu}}$ and the investigation of the Lorentz structure of the leptonic weak charged current. Through combination of its purely-leptonic nature - permitting rigorous theoretical analysis- 

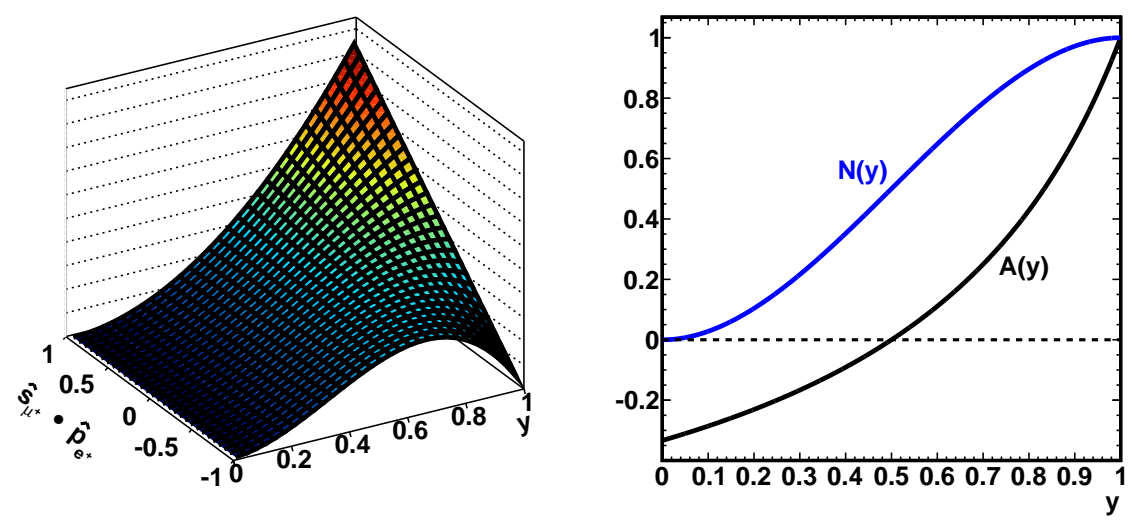

Figure 5: Left: Relative probability in arbitrary units of the emitted positron in ordinary muon decay as a function of the muon spin direction $\hat{s_{\mu}}$ and the emitted positron reduced energy $y$ for the $V$-A weak interaction. Right: Projections of the relative number, $N(y)$, and asymmetry, $A(y)$, vs. positron reduced energy.

analysis - the decay $\mu^{+} \rightarrow e^{+} \nu_{e} \overline{\nu_{\mu}}$ is the quintessential test of the $V$ - $A$ structure of the weak interaction.

The measurable observables of emitted positrons in $\mu^{+} \rightarrow e^{+} \nu \bar{\nu}$ decay are the positron energy and directional distributions, shown in Fig. 5, and the positron polarization. The $V$ - $A$ interaction dictates the precise form of the energyangle distribution and the polarization observables which include the parity non-conserving angular asymmetry and longitudinal polarization. In general the presence of non- $V$ - $A$ currents would change these energy, angle and polarization distributions.

Based upon a general current-current interaction with possible scalar, pseudoscalar, tensor, vector and axial terms the positron energy, angle and polarization distributions may be parameterized by nine parameters (see Refs. [15, 16, 50]). The differential probability for emitting a positron with a reduced energy $y$ at an angle $\theta$ from the muon spin axis is given by

$$
\frac{d^{2} \Gamma}{d y d \cos \theta}=\frac{1}{4 \pi^{3}} m_{\mu} W_{e \mu}^{4} G_{F}^{2} \sqrt{y^{2}-y_{0}^{2}}\left(F_{I S}(y)+P_{\mu} \cos \theta F_{A S}(y)\right)
$$

where $P_{\mu}=\left|\vec{P}_{\mu}\right|$ is the muon polarization, $W_{e \mu}=\left(m_{\mu}^{2}+m_{e}^{2}\right) / 2 m_{\mu}=52.8 \mathrm{MeV}$ is the maximum positron energy, $y=E_{e} / W_{e \mu}$ is the reduced energy, and $y_{o}=$ $m_{e} / W_{e \mu}=9.7 \times 10^{-3}$. The y-dependent functions

$$
\begin{gathered}
F_{I S}(y)=y(1-y)+\frac{2}{9} \rho\left(4 y^{2}-3 y-y_{o}^{2}\right)+\eta y_{o}(1-y) \\
F_{A S}(y)=\frac{1}{3} \xi \sqrt{y^{2}-y_{o}^{2}}\left\{1-y+\frac{2}{3} \delta\left[4 y-3+\left(\sqrt{1-y_{o}^{2}}-1\right)\right]\right\}
\end{gathered}
$$


describe the isotropic and anisotropic parts of the energy spectrum. They are governed by the decay parameters $\rho, \eta, \xi$, and $\delta$ - conventionally termed the Michel parameters. For unpolarized muons, the two parameters $\rho$ and $\eta$ completely determine the energy distribution with $\rho$ governing the high-energy part and $\eta$ governing the low-energy part of the spectrum 7 For polarized muons, the two parameters $\xi$ and $\delta$ additionally determine the angular distribution with $\xi$ governing the energy-integrated positron asymmetry and $\delta$ governing the energy dependence of this angular asymmetry. The standard model values of the Michel parameters are $\rho=0.75, \eta=0.0, \xi=1.0$ and $\delta=0.75$.

The energy-angle dependent positron polarization vector $\vec{P}_{e}(y, \theta)$ may be decomposed into a longitudinal component $P_{L}$ and two transverse components $P_{T 1}, P_{T 2}$ according to

$$
\vec{P}_{e}(y, \theta)=P_{L} \cdot \hat{z}+P_{T 1} \cdot\left(\hat{z} \times \hat{P}_{\mu}\right) \times \hat{z}+P_{T 2} \cdot\left(\hat{z} \times \hat{P}_{\mu}\right)
$$

where $\hat{z}$ and $\hat{P}_{\mu}$ are unit vectors along the positron momentum and the muon ${ }_{505}$ polarization, respectively. The transverse polarization $P_{T 1}$ lies in the decay plane of the positron momentum and the muon spin while the transverse polarization $P_{T 2}$ lies along the perpendicular to this decay plane. Note that the transverse polarization $P_{T 1}$ is a (time-reversal) T-conserving observable while the transverse polarization $P_{T 2}$ is a T-violating observable.

The energy-angle dependence of the longitudinal polarization $P_{L}$ is given by

$$
P_{L}(y, \theta)=\frac{F_{I P}(y)+P_{\mu} \cos \theta F_{A P}(y)}{F_{I S}(y)+P_{\mu} \cos \theta F_{A S}(y)} .
$$

The two additional y-dependent functions are

$$
\begin{aligned}
& F_{I P}(y)=\frac{1}{54} \sqrt{y^{2}-y_{o}^{2}}\left\{9 \xi^{\prime}\left(-2 y+2+\sqrt{1-y_{o}^{2}}\right)+4 \xi\left(\delta-\frac{3}{4}\right)\left(4 y-4+\sqrt{1-y_{o}^{2}}\right)\right\} \\
& F_{A P}(y)=\frac{1}{6}\left\{\xi^{\prime \prime}\left(2 y^{2}-y-y_{o}^{2}\right)+4\left(\rho-\frac{3}{4}\right)\left(4 y^{2}-3 y-y_{o}^{2}\right)+2 \eta^{\prime \prime}(1-y) y_{o}\right\}
\end{aligned}
$$

510 and involve two further decay parameters $-\xi^{\prime}$ and $\xi^{\prime \prime} 8$ Neglecting the positron mass and radiative corrections, the standard model values of the decay parameters $\xi^{\prime}=\xi^{\prime \prime}=1.0$ yield an energy-angle independent longitudinal polarization $P_{L}=1$. by

The T-conserving polarization $P_{T 1}$ and its energy-angle dependence is given

$$
P_{T 1}(y, \theta)=\frac{P_{\mu} \sin \theta F_{T 1}(y)}{F_{I S}(y)+P_{\mu} \cos \theta F_{A S}(y)}
$$

\footnotetext{
${ }^{7}$ The contribution of $\eta$ is order $y_{o}$ and consequently very small.

${ }^{8} \mathrm{An}$ additional parameter $\eta^{\prime \prime}$ also enters $F_{A P}(y)$ but its role is highly suppressed by the factor $y_{0}$.
} 
and the T-violating polarization $P_{T 2}$ and its energy-angle dependence is given by

$$
P_{T 2}(y, \theta)=\frac{P_{\mu} \sin \theta F_{T 2}(y)}{F_{I S}(y)+P_{\mu} \cos \theta F_{A S}(y)} .
$$

The two additional y-dependent functions are

$$
\begin{gathered}
F_{T 1}(y)=\frac{1}{12}\left\{-2\left[\xi^{\prime \prime}+12\left(\rho-\frac{3}{4}\right)\right](1-y) y_{o}-3 \eta\left(y^{2}-y_{o}^{2}\right)+\eta^{\prime \prime}\left(-3 y^{2}+4 y-y_{o}^{2}\right)\right\} \\
F_{T 2}(y)=\frac{1}{3} \sqrt{y^{2}-y_{o}^{2}}\left[3 \frac{\alpha^{\prime}}{A}(1-y)+2 \frac{\beta^{\prime}}{A} \sqrt{1-y_{o}^{2}}\right]
\end{gathered}
$$

The T-conserving polarization $P_{T 1}$ and its energy-angle dependence are determined by the decay parameters $\eta$ and $\eta^{\prime \prime}$ and the T-violating polarization $P_{T 2}$ and its energy-angle dependence are determined by the decay parameters $\alpha^{\prime} / A$ and $\beta^{\prime} / A$. The standard model values of these parameters are all zero; $\eta=\eta^{\prime \prime}=\alpha^{\prime} / A=\beta^{\prime} / A=0$. They yield-when neglecting the positron mass and radiative corrections - an energy-angle independent transverse polar-

520 ization $P_{T 1}=P_{T 2}=0$. On accounting for positron mass effects the average T-conserving transverse polarization component is $\left\langle P_{T 1}\right\rangle=-3 \times 10^{-3}$ [51].

The standard model and measured values of the decay parameters are summarized in Table 2. Until recently our knowledge of the muon decay parameters was based on experiments from twenty, thirty and forty years ago. For example, the best determination of the energy spectrum parameter $\rho$ was due to Derenzo et al. [52] and the best determinations of the angular distribution parameters $\xi$ and $\delta$ were due to Balke et al. [53, Beltrami et al. 54 and Jodidio et al. 55 .

The past decade has seen a substantial improvement in the experimental knowledge of the decay parameters of both the $\mu^{+} \rightarrow e^{+} \nu \bar{\nu}$ energy-angle distri530 bution and the various polarization observables. A precision measurement of the positron energy-angle distribution in polarized muon decay was conducted by the TWIST collaboration [56, 57] at the TRIUMF cyclotron. It led to roughly a factor ten improvement in the determination of the decay parameters $\rho, \xi$ and $\delta$. Other precision measurements of both the transverse polarization [51] and the ${ }_{535}$ longitudinal polarization [58 of the decay positrons were recently conducted at PSI. They have improved our knowledge of the longitudinal polarization parameter $\xi^{\prime \prime}$ by roughly a factor of ten and the transverse polarization parameters by roughly a factor of three.

\subsection{TWIST experiment}

TWIST - the TRIUMF Weak Interaction Symmetry Test experiment [56, 57 - was designed to measure with unprecedented accuracy the energy-angle distribution of decay positrons from polarized muons. The setup-including the muon beam, stopping target and positron spectrometer - is shown in Fig. 6. The experiment involved stopping a highly polarized muon beam in a very

${ }_{545}$ thin target foil and detecting the resulting decay positrons in a low mass, large acceptance, high resolution magnetic spectrometer. 


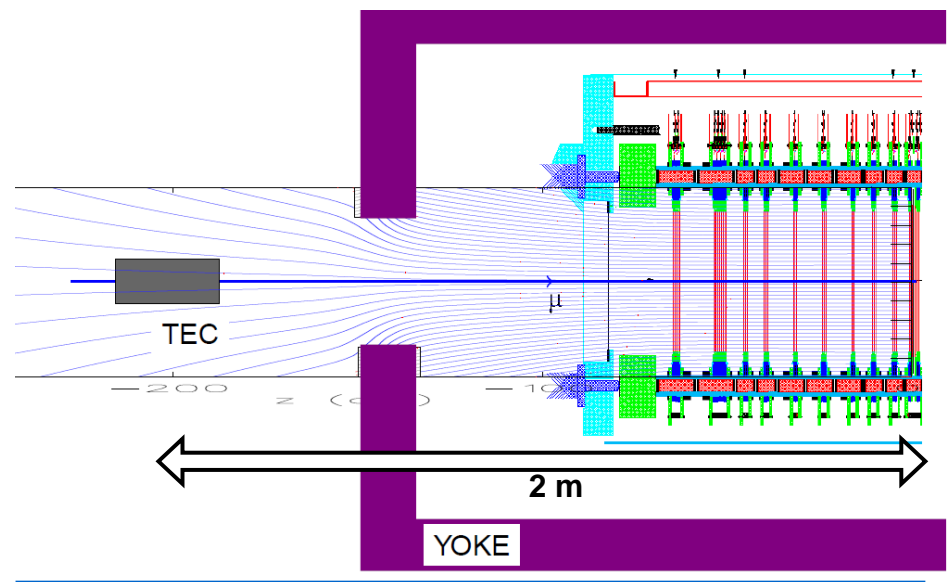

Figure 6: Cross section view of the TWIST experiment indicating the muon beam, time expansion chambers (TECs), beam scintillator, planar drift and proportional chambers, stopping target, and the iron yoke and the magnetic field of the cylindrical superconducting magnet. Figure courtesy TWIST collaboration.

The experiment utilized a $0.7 \%$ momentum bite, surface muon beam with a near-100\% polarization at typical rates of several $\mathrm{kHz}$. To fully characterize and periodically monitor the beam properties the beamline incorporated two custom time expansion chambers (TECs). Each TEC contained a separate horizontal and vertical drift region to measure the horizontal and vertical trajectories of individual beam particles. The TECs were constructed with ultra-thin entrance and exit windows and filled with low pressure $(80$ mbar $)$ gas.

The beam entered the spectrometer through a thin scintillator and a gas 555 degrader. A feedback system adjusted the degrader's helium / $\mathrm{CO}_{2}$ gas mixture to account for environmental changes and maintain centering of the stopping distribution in the target foils.

The magnetic spectrometer permitted the measurement of both the beam muons and the decay positron. The setup comprised a symmetric array of planar drift chambers and planar proportional chambers along the common beam and spectrometer axis. The drift chambers enabled the precision determination of the momentum-angle coordinates from the helical trajectories of the decay positrons. The proportional chambers rendered the $\mu / e$ particle discrimination via the above-threshold time interval of the resulting chamber signals.

565 The low-mass chambers were constructed with ultra-thin windows and filled with slow-drift gas to optimize position resolution. The support structure was constructed from low-thermal expansion ceramics to reduce the effects of temperature fluctuations on spectrometer resolution.

The experiment utilized two different stopping targets; a $31 \mu \mathrm{m}$ thickness, high-purity silver foil and a $72 \mu \mathrm{m}$ thickness, high-purity aluminum target. To optimize the detector resolution the target foils were employed as the window material separating the two central drift chambers of the magnetic spectrometer. 
A cylindrical superconducting magnet with symmetric upstream and downstream holes provided a uniform magnetic field along the beam axis. The field uniformity in the tracking region was a few parts-per-thousand and the field stability was continuously monitored by NMR probes. The field was both mapped with a movable Hall probe and calculated using a finite element analysis. The track reconstruction used the calculated field distribution anchored by the Hall probe measurements.

580 The experiment accumulated large datasets of decay positrons from each target as well as dedicated measurements of beam properties and other systematics uncertainties. Both experimental data and simulated data were stored in a common data format and processed with a common analysis code.

The analysis involved the reconstruction of the helical particle tracks from the raw TDC data and the construction of the positron momentum-angle (p- $\theta$ ) distribution from the track parameters. A sophisticated simulation of particle interactions, detector geometry, and data readout incorporated: the TEC measurements of beam particle trajectories, the discontinuous nature of chamber ionization, a Garfield calculation of the position-dependent drift velocities, an 590 OPERA calculation of the magnetic field distribution, and rate-dependent effects of overlapping tracks. The simulated $\mathrm{p}-\theta$ coordinates of decay positrons included full radiative corrections to order $\mathcal{O}\left(\alpha^{2}\right)$ and leading-log radiative corrections to order $\mathcal{O}\left(\alpha^{3}\right)$.

Importantly the measured and simulated p- $\theta$ distributions are functions of both the muon decay parameters and the $\mathrm{p}-\theta$ dependence of the spectrometer response function and the track reconstruction efficiency. Therefore the fitting of p- $\theta$ distributions is used to extract only the differences between the simulated values and the measured values of the decay parameters, i.e. $\Delta \rho, \Delta \xi$ and $\Delta \delta$. This "relative" method facilitated a blind analysis where the exact values of the decay parameters were not known to the collaboration until the completion of the data analysis.

The final results for the Michel parameters extracted from the combined analysis of the experimental data and the simulated data were

$$
\begin{aligned}
\rho & =0.74977 \pm 0.00012 \text { (stat) } \pm 0.00023 \text { (syst) } \\
\delta & =0.75049 \pm 0.00021 \text { (stat) } \pm 0.00073 \text { (syst) } \\
P_{\mu}^{\pi} \xi & =1.00084 \pm 0.00029 \text { (stat) } \pm{ }_{0.00063}^{0.00165} \text { (syst). }
\end{aligned}
$$

Note that the decay parameter $\xi$ and the muon polarization in pion decay $P_{\mu}^{\pi}$ 605 are experimentally inseparable. Contrary to $\rho$ and $\delta$ the uncertainty in $P_{\mu}^{\pi} \xi$ is dominated by muon depolarization effects including the spectrometer-beam alignment and spectrometer fringe fields.

\subsection{Measurement of the positron longitudinal polarization}

A measurement of the positron longitudinal polarization $P_{L}$ in muon decay was recently reported by Prieels et al. [58]. The experimental setup was configured to measure the longitudinal polarization near the positron energy endpoint and opposite to the muon spin direction. This kinematical region is uniquely 
sensitive to $\xi^{\prime \prime}$, the decay parameter that characterizes the angle-energy dependence of $P_{L}$. A polarization of $P_{L} \neq 1$ for high-energy, backward-angle positrons would signal a departure of this decay parameter from the SM value $\xi^{\prime \prime}=1$.

The experiment utilized a high intensity, longitudinally polarized, surface muon beam at PSI. The incident muons were stopped in either an aluminum target or a sulfur target that were located in a $0.1 \mathrm{~T}$ longitudinal holding field. In aluminum the initial muon polarization is only weakly depolarized thus yielding ${ }_{620}$ a large time-averaged muon polarization along the polarimeter axis. In sulfur the initial polarization is more strongly depolarized thus yielding a small timeaveraged muon polarization along the polarimeter axis.

The detection system consisted of a small acceptance, high precision, cylindrical spectrometer followed by a positron polarimeter. The spectrometer was orientated to accept only decay positrons that were emitted directly opposite to the muon polarization direction. It consisted of three parts: a filter, tracker and lens. The filter comprised an initial cylindrical magnet with collimators arranged to transmit only high energy positrons. The tracker comprised a superconducting cylindrical magnet with three planes of position-sensitive silicon detectors

630 to measure the trajectories and determine the energies of decay positrons. The lens comprised a final cylindrical magnet that focused the decay positrons into parallel trajectories at the polarimeter entrance.

The polarimeter utilized the Bhabha scattering $e^{+} e^{-} \rightarrow e^{+} e^{-}$and in-flight annihilation $e^{+} e^{-} \rightarrow \gamma \gamma$ of decay positrons on polarized electrons in magnetized ${ }_{635}$ foils. The cross section for Bhabha scattering and in-flight annihilation is different for the two orientations with the $e^{+} e^{-}$-spins parallel and the $e^{+} e^{-}$-spins anti-parallel. These spin-dependent cross sections thus induce a signal rate from scattering and annihilation that depends on $P_{L}$.

The polarimeter employed (see Fig. 7) a double layer of oppositely magnetized foils that were orientated with their magnetization axes at $\pm 45^{\circ}$ angles to the spectrometer axis. The $\pm 45^{\circ}$ configuration yielded an electron polarization along the spectrometer axis of roughly $5 \%$. A series of wire chambers and plastic scintillators at the location of the foils was used to discriminate the struck foil and determine the scattering / annihilation vertex. A bismuth germanate ${ }_{645}$ detector array was used to measure the energies and position coordinates of the annihilation $\gamma$-rays or the scattered $e^{+} e^{-}$. Asymmetries in rates of annihilation and scattering were investigated by reversing the foil magnetization and inverting the $\pm 45^{\circ}$ foil orientation. The measured energies and opening angles of $\gamma$-rays or $e^{+} e^{-}$pairs - along with the measured energy of the decay positron in the magnetic spectrometer - over determined the reaction kinematics and allowed for backgrounds suppression.

The analysis involved constructing the super-ratio

$$
s_{\alpha}=\frac{r_{\alpha}^{+}-r_{\alpha}^{-}}{r_{\alpha}^{+}+r_{\alpha}^{-}}
$$

where $r_{\alpha}^{ \pm}$is the event ratio from the two foils and the superscript \pm denotes the two magnetization orientations. The subscript $\alpha$ distinguishes the eight 


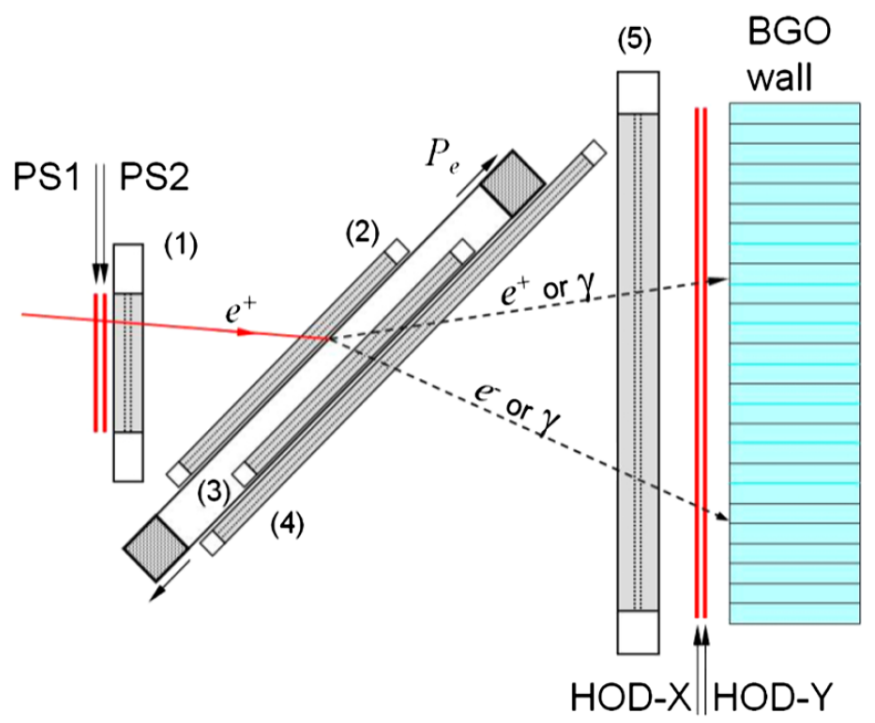

Figure 7: Schematic diagram of the double foil positron polarimeter in the Prieels et al. experiment [58. It shows the two oppositely-magnetized foils, the arrangement of wire chambers and plastic scintillators that discriminate between $\gamma$-ray and $e^{+} e^{-}$events and determine their vertex coordinates, and the bismuth germanate array. Figure courtesy Prieels et al.

measurement configurations consisting of (i) \pm 45 -degree foil orientations, (ii) aluminum / sulfur targets, and (iii) annihilation / scattering events. The superratios are proportional to the longitudinal polarization $P_{L}$ of the decay positrons. Moreover the use of $s_{\alpha}$ cancels the effects of differing detection efficiencies for scattering / annihilation events from the two foils.

The key characteristic of $\xi^{\prime \prime}$ differing from unity is the introduction of an energy-dependent longitudinal polarization $P_{L}$ of the backward-emitted decay positrons. Consequently, a value of $\xi^{\prime \prime} \neq 1$ can be identified through a comparatively strong energy dependence of $s_{\alpha}$ for the polarization-preserving $\mathrm{Al}$ target with a comparatively weak energy dependence of $s_{\alpha}$ for the polarizationdestroying $\mathrm{S}$ target. No evidence of such an effect was observed in the comparison of the measured super-ratios for the two target materials.

A combined fit to the super-ratio data for all experimental configurations yielded the value 9

$$
\xi^{\prime \prime}=0.981 \pm 0.045(\text { stat }) \pm 0.003 \text { (syst) }
$$

\footnotetext{
${ }^{9}$ The fitting procedure incorporated energy-independent attenuation factors that empirically account for globally lower than predicted values for the analyzing powers for the double foil arrangement. Although such effects as a smaller than expected magnetization and a larger than expected background were investigated, the cause of the attenuator factors was not convincingly established.
} 


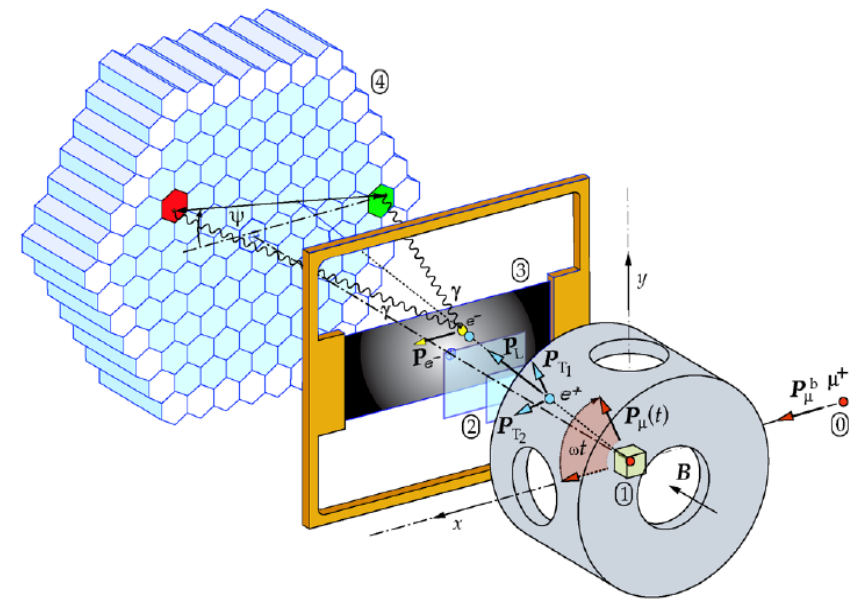

Figure 8: Schematic diagram of the Danneberg et al. experiment 51 indicating the longitudinally polarized muon beam, beryllium stopping target, transverse precession field, magnetized iron-cobalt foil and the bismuth germanate detector array. Figure courtesy Danneberg et al.

This result represents a ten-fold improvement over earlier experimental work and is consistent with the SM prediction $\xi^{\prime \prime}=1.0$.

\subsection{Measurement of the positron transverse polarization}

Danneberg et al. [51] have recently reported a measurement of the transverse polarization of the positrons emitted in the $\mu \rightarrow e \nu \bar{\nu}$ decay. In the limit of vanishing positron mass the $V$-A theory implies neither a T-conserving transverse polarization (i.e. $P_{T 1}=0$ ) nor a T-violating transverse polarization (i.e. $\left.P_{T 2}=0\right)$ for the emitted positrons. After accounting for positron mass effects the average T-conserving transverse polarization in $V$-A theory is about $\left\langle P_{T 1}\right\rangle=-3 \times 10^{-3}$.

The experiment was conducted at PSI. The experimental setup - shown in Fig. 8 -incorporated a polarized muon beam, beryllium stopping target and positron polarimeter. To optimize the sensitivity to $P_{T}$ the polarimeter was orientated at a 90-degree angle to the muon polarization axis.

The high-rate muon beam possessed the cyclotron radio frequency time structure that consisted of pulses at 20 ns intervals with 4 ns durations. A $0.37 \mathrm{~T}$ transverse magnetic field was used to maintain the ensemble polarization of multiple muon stops via the matching of the Larmor precession period to the $20 \mathrm{~ns}$ pulse period. The $4 \mathrm{~ns}$ pulse duration of beam particles limited the ensemble polarization of stopped muons to roughly $80 \%$.

The polarimeter employed the detection of $\gamma$-ray pairs from the in-flight $e^{+} e^{-}$annihilation of the decay positrons on the polarized electrons in a magnetized iron-cobalt foil. The resulting angular distribution of gamma-ray pairs is 
correlated with the relative orientation of the $e^{+}-e^{-}$polarization axes. The time and position coordinates of the positron annihilation on the magnetized foil were measured by combination of plastic scintillators and planar drift chambers. The energy and position coordinates of the coincident $\gamma$-rays were measured using a bismuth germanate (BGO) detector array.

The Larmor precession of stopped muons is important in understanding the experimental signature of transverse polarization. This precession would cause the corresponding rotation of any non-zero components of the positron transverse polarization. Consequently, the angular distribution of the annihilation $\gamma$-rays would likewise rotate at the Larmor frequency, with an amplitude and a phase that is governed by the transverse polarization $\left(P_{T 1}, P_{T 2}\right)$. By flipping the magnetization of the polarimeter foils or reversing the direction of muon precession, the associated signal of transverse polarization could be distinguished from other systematic effects that might induce periodic signals in the $\gamma$-ray time distributions 10

The data analysis involved constructing time distributions $N_{i j}(t)$ of the $\gamma$-ray coincidences in the BGO detector elements $(i, j)$ as a function of the angle $\psi$ between the $\gamma$-ray pair plane and the horizontal magnetization plane. A non-zero transverse polarization would impart on $N_{i j}(t)$ a $\psi$-dependent sinusoidal signal with the Larmor frequency and an overall amplitude and phase determined by $\left(P_{T 1}, P_{T 2}\right)$.

The experiment recorded about $10^{6}$ annihilation $\gamma$-ray pairs from decay positrons and permitted measurement of $P_{T 1}$ and $P_{T 2}$ for positron energies 10-50 MeV. A Monte Carlo simulation was performed to determine the dependence of the transverse polarizations on each decay parameter. A fit was then performed of the Monte Carlo polarizations to the measured polarizations to determine the "best fit" values for $\eta, \eta^{\prime \prime}, \alpha^{\prime} / A$ and $\beta^{\prime} / A$. The procedure yielded values of

$$
\begin{aligned}
\eta & =(71 \pm 37 \pm 5) \times 10^{-3} \\
\eta^{\prime \prime} & =(100 \pm 52 \pm 6) \times 10^{-3}
\end{aligned}
$$

for the T-conserving polarization component $P_{T 1}$ and

$$
\begin{aligned}
\alpha^{\prime} / A & =(-3.4 \pm 21.3 \pm 4.9) \times 10^{-3} \\
\beta^{\prime} / A & =(-0.5 \pm 7.8 \pm 1.8) \times 10^{-3}
\end{aligned}
$$

for the T-violating polarization component $P_{T 2}$. Using these best-fit values for the decay parameters the authors then derived corresponding values for energy720 averaged polarizations of

$$
\begin{gathered}
\left\langle P_{T 1}\right\rangle=(6.3 \pm 7.7 \pm 3.4) \times 10^{-3} \\
\left\langle P_{T 2}\right\rangle=(-3.7 \pm 7.7 \pm 3.4) \times 10^{-3}
\end{gathered}
$$

\footnotetext{
${ }^{10}$ Specifically, "off-axis" positron annihilation can also induce sinusoidal variations in the $\gamma$-ray time distribution. This off-axis signal was used for polarimeter calibration.
} 
The determinations of the transverse polarizations and their decay parameters represent a three-fold improvement over previous experimental work. The results are consistent with zero transverse polarization and the SM values for the decay parameters, $\eta=\eta^{\prime \prime}=\alpha^{\prime} / A=\beta^{\prime} / A=0$.

\subsection{Global analysis of decay parameters and theoretical implications}

A common representation for the matrix element of the most general, Lorentz invariant, derivative-free, current-current interaction is based on definite electron and muon chiralities

$$
M \propto \sum_{\gamma=S, V, T}^{\epsilon, \mu=L, R} g_{\epsilon \mu}^{\gamma}\left\langle\bar{e}_{\epsilon}\left|\Gamma_{\gamma}\right| \nu_{e}\right\rangle\left\langle\overline{\nu_{\mu}}\left|\Gamma^{\gamma}\right| \mu_{\mu}\right\rangle
$$

where $\Gamma^{S}, \Gamma^{V}, \Gamma^{T}$ represent the possible (scalar-pseudoscalar, vector-axial, tensor) interactions between a left / right handed muon and a left / right handed electron. The parameters $g_{\epsilon \mu}^{\gamma}$ are the ten associated complex coupling constants of the current-current interaction terms $\left(g_{R R}^{T}\right.$ and $g_{L L}^{T}$ are identically zero). In the case of the $V$-A weak interaction, the coupling $g_{L L}^{V}$ is unity and all the remaining couplings are exactly zero.

The nine decay parameters from ordinary decay $\mu^{+} \rightarrow e^{+} \nu \bar{\nu}$, and one additional parameter from radiative decay $\mu^{+} \rightarrow e^{+} \nu \bar{\nu} \gamma$, appear insufficient at first glance to unambiguously determine the ten independent complex couplings $g_{\epsilon \mu}^{\gamma}{ }^{11}$ Therefore the global analyses of measured decay parameters involve determining values for smaller sets of intermediate bilinear combinations of complex couplings. The possible limits on non- $V$ - $A$ interactions - i.e. all couplings $g_{\epsilon \mu}^{\gamma}$ excepting $g_{L L}^{V}$-are then derived from the values determined for the bilinear combinations.

Sets of intermediate combinations of complex couplings for parameterizing the constraints obtained from decay parameters have been introduced by Kinoshita and Sirlin [50] and Fetscher, Gerber and Johnson [59]. For example, the scheme of Fetscher et al. incorporates the maximum number of positive semidefinite bilinear combinations of complex couplings $g_{\epsilon \mu}^{\gamma}$. Of ten parameters, the four bilinears

$$
\begin{gathered}
Q_{L L}=\frac{1}{4}\left|g_{L L}^{S}\right|^{2}+\left|g_{L L}^{V}\right|^{2}, \\
Q_{L R}=\frac{1}{4}\left|g_{L R}^{S}\right|^{2}+\left|g_{L R}^{V}\right|^{2}+3\left|g_{L R}^{T}\right|^{2}, \\
Q_{R L}=\frac{1}{4}\left|g_{R L}^{S}\right|^{2}+\left|g_{R L}^{V}\right|^{2}+3\left|g_{R L}^{T}\right|^{2}, \\
Q_{R R}=\frac{1}{4}\left|g_{R R}^{S}\right|^{2}+\left|g_{R R}^{V}\right|^{2}
\end{gathered}
$$

\footnotetext{
${ }^{11}$ One coupling is contrained by normalization thus leaving the Fermi constant $G_{F}$ and eighteen additional independent parameters to be experimentally determined.
} 

other couplings zero. The world data on decay parameters are consistent with the $V$-A charged current weak interaction to levels of $10^{-3}-10^{-4}$ in many cases.

Table 2: Compilation of the most recent determinations of the nine decay parameters in ordinary muon decay and the additional $\bar{\eta}$ decay parameter in radiative muon decay. With the exception of the ordinary decay parameter $\xi^{\prime}$ and the radiative decay parameter $\bar{\eta}$, we give the results of the most recent precision measurements. For $\xi^{\prime}$ and $\bar{\eta}$ we give the world averages compiled in Ref. 43] (the average for $\xi^{\prime}$ is dominated by the measurement of Burkard et al. [60] and the average for $\bar{\eta}$ is dominated by the measurement of Eichenberger et al. [61]).

\begin{tabular}{cccc}
\hline Parameter & Measured value & SM value & Ref. \\
\hline$\rho$ & $0.74977 \pm 0.00012 \pm 0.00023$ & 0.75 & {$[56]$} \\
$\delta$ & $0.75049 \pm 0.00021 \pm 0.00027$ & 0.75 & {$[56]$} \\
$P_{\mu}^{\pi} \xi$ & $1.00084 \pm 0.00029 \pm_{0.00063}^{0.00165}$ & 1.0 & {$[57]$} \\
$\xi^{\prime}$ & $1.00 \pm 0.04$ & 1.0 & {$[43]$} \\
$\xi^{\prime \prime}$ & $0.981 \pm 0.045 \pm 0.003$ & 1.0 & {$[58]$} \\
$\eta$ & $(71 \pm 37 \pm 5) \times 10^{-3}$ & 0.0 & {$[51]$} \\
$\eta^{\prime \prime}$ & $(100 \pm 52 \pm 6) \times 10^{-3}$ & 0.0 & {$[51]$} \\
$\alpha^{\prime} / A$ & $(-3.4 \pm 21.3 \pm 4.9) \times 10^{-3}$ & 0.0 & {$[51]$} \\
$\beta^{\prime} / A$ & $(-0.5 \pm 7.8 \pm 1.8) \times 10^{-3}$ & 0.0 & {$[51]$} \\
$\bar{\eta}$ & $0.02 \pm 0.08$ & 0.0 & {$[43]$} \\
\hline
\end{tabular}

A global analysis of decay parameters has recently been performed by the TWIST collaboration [56. It followed the procedure of Gagliardi, Tribble and Williams 62 but updated their input values for decay parameters with the final results of the TWIST experiment 12 The procedure involved determining the joint probability distributions for a hybrid set of nine bilinears of coupling $g_{\epsilon \mu}^{\gamma}$ from the muon decay parameters via a Monte Carlo procedure (see Ref. 63] for 755 details). From these results, they then derived limits on the various couplings $g_{\epsilon \mu}^{\gamma}$. Their results for the bilinears $Q_{e \mu}$ and the couplings $g_{\epsilon \mu}^{\gamma}$ are reproduced in Table 3

Of special interest are the upper limits derived on the bilinear sums $Q_{R}^{\mu} \equiv$ $Q_{R R}+Q_{L R}$ and $Q_{R}^{e} \equiv Q_{R R}+Q_{R L}$ A non-zero value of $Q_{R}^{\mu}$ would signal a weak interaction contribution from a right-handed muon current and a non-zero value of $Q_{R}^{e}$ would signal a weak interaction contribution from a right-handed electron current. The decay parameter $\xi$ of the positron angular distribution is particularily sensitive to $Q_{R}^{\mu}$ and the decay parameter $\xi^{\prime}$ of the positron

${ }^{12}$ This global analysis precedes the recent measurement of $\xi^{\prime \prime}$ by Prieels et al. 
Table 3: Results for the experimental limits on the bilinear quantities $Q_{\epsilon \mu}$ and the coupling constants $g_{\epsilon \mu}^{\gamma}$ of the general current-current interaction derived from the global analysis of the muon decay parameters [56]. In $V$-A theory the bilinear $Q_{L L}$ and coupling $g_{L L}^{V}$ are unity and all other bilinears and coupling constants are exactly zero. Note the limits on $\left|g_{L L}^{S}\right|$ and $\left|g_{L L}^{V}\right|$ are derived from inverse muon decay, $\nu_{\mu} e^{-} \rightarrow \nu_{e} \mu^{-}$.

$$
\begin{array}{cccc}
Q_{R R}<3.0 \times 10^{-4} & Q_{L R}<6.3 \times 10^{-4} & Q_{R L}<0.044 & Q_{L L}>0.955 \\
& & & \\
\left|g_{R R}^{S}\right|<0.035 & \left|g_{L R}^{S}\right|<0.050 & \left|g_{R l}^{S}\right|<0.420 & \left|g_{L L}^{S}\right|<0.550 \\
\left|g_{R R}^{V}\right|<0.017 & \left|g_{L R}^{V}\right|<0.023 & \left|g_{R L}^{V}\right|<0.105 & \left|g_{L L}^{V}\right|>0.960 \\
\left|g_{R R}^{T}\right| \equiv 0 & \left|g_{L R}^{T}\right|<0.015 & \left|g_{R L}^{T}\right|<0.1050 & \left|g_{L L}^{T}\right| \equiv 0
\end{array}
$$

longitudinal polarization is particularily sensitive to $Q_{R}^{e}$. The global analysis 765 yielded limits on contributions from right-handed muon interactions of $Q_{R}^{\mu}<$ $8.2 \times 10^{-4}$ and right-handed electron interactions of $Q_{R}^{e}<0.044$.

Danneberg et al. and Prieels et al. also discuss limits on right-handed currents involving specific exotic scalar, vector and tensor interactions. For example, the decay parameters $\alpha^{\prime} / A$ and $\beta^{\prime} / A$ derived from the transverse polarization $P_{T 2}$ are uniquely sensitive to T-violating contributions in purely leptonic interactions. Danneberg et al. obtained a limit on possible T-violating scalar interactions between right-handed charged leptons currents of $\operatorname{Im}\left(g_{R R}^{s}\right)=$ $(5.2 \pm 14.0 \pm 2.4) \times 10^{-3}$.

Muon decay is particularly valuable in imposing constraints on various leftright symmetric (LRS) extensions of the standard model electroweak interaction 64. Such models introduce a new $V+A$ interaction coupling to right-handed currents that partners the known $V$ - $A$ interaction coupling to left-handed currents in order to restore parity conservation at high energies. In LRS models the $V+A$ and $V$-A interactions are mediated by $W_{R}$ and $W_{L}$ gauge bosons with couplings constants $g_{R}$ and $g_{L}$. A mass mixing angle $\zeta$ and CP-violating phase $\omega$ together determine the relation between the weak eigen-states $W_{L / R}$ and the mass eigen-states $W_{1 / 2}$

$$
\begin{gathered}
W_{L}=W_{1} \cos \zeta+W_{2} \sin \zeta \\
W_{R}=e^{i \omega}\left(-W_{1} \sin \zeta+W_{2} \cos \zeta\right)
\end{gathered}
$$

with low-energy parity violation emerging when the $W_{R}$-boson mass exceeds the $W_{L}$-boson mass. In manifest LRS models the two couplings are equal and in generalized LRS models the two coupling are distinct.

A variety of approaches - from direct searches for $W_{R}$-boson production at pp colliders to setting limits on $W_{R^{-}}$boson virtual contributions in $K^{0}-\bar{K}^{0}$ mixing, $\beta$-decay and muon decay - have been used in the investigation of the 780 various LRS extensions of the standard model (for further details see the reviews [43, 65]). The results from pp colliders and $K^{0}-\bar{K}^{0}$ mixing set impressive 


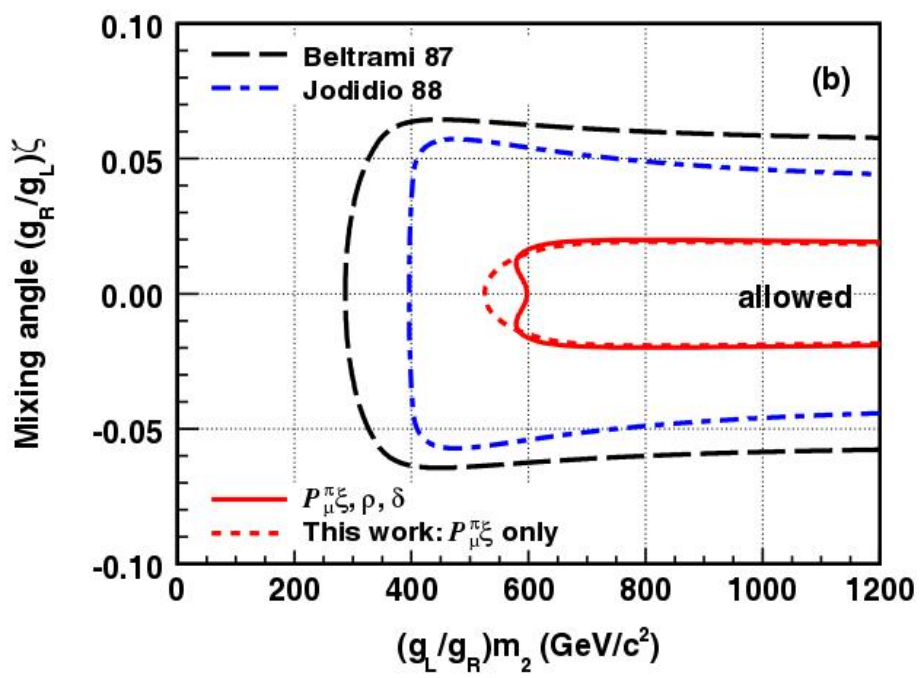

Figure 9: Allowed region of $W_{2}$ boson mass versus mass mixing angle $\zeta$ in the generalized leftright symmetric model. Shown are the limits derived from the recent TWIST measurement of $P_{\mu} \xi$ and the earlier results from Beltrami et al. [54] and Jodidio et al. 55].

lower bounds on $W_{R}$-boson masses while results from unitarity tests that utilize $0^{+} \rightarrow 0^{+}$nuclear $\beta$-decay set impressive bounds on $W_{L}-W_{R}$ mixing. However, unlike these processes the decay of muons is purely leptonic and essentially free of any assumptions concerning the CKM matrix elements of the hypothetical $W_{R}$ boson (many analyses assume a $W_{R}$ boson with standard model-like couplings and CKM matrix elements). Consequently, the TWIST result for $P_{\mu} \xi$ [57] yields complementary and restrictive limits on the parameter space of the generalized LRS model. Their limits on $g_{L} / g_{R} m_{2}$ versus $g_{L} / g_{R} \zeta$ are reproduced in Fig. 9 ,

\section{Charged Lepton Flavor Violating decays}

\subsection{Lepton flavor and physics beyond the standard model}

Once a muon, always a muon - or at least the flavor of a muon seems to be preserved. The conservation of a "muon" flavor was not expected when 795 this particle was discovered, nor does it associate a conserved quantity with a fundamental symmetry, as required by Noether's theorem. But, following nonobservations of its violation over decades of trials in reactions such as the simple decay mode $\mu \rightarrow e \gamma$, it emerged as a standard model foundational statement; a muon cannot become an electron.

In a two-neutrino (later, three) world of massless neutrinos, the transformation of muon flavor to electron flavor (or tau flavor) is strictly forbidden. The conservation of separate $e-, \mu-$, and $\tau$-type lepton numbers holds; that 


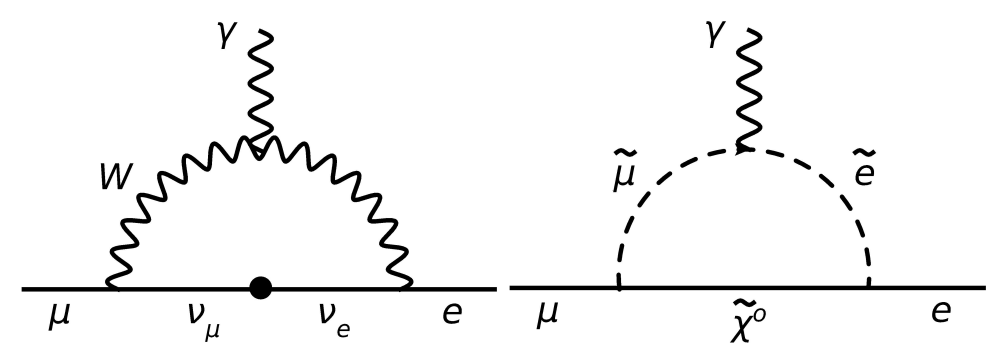

Figure 10: a) The standard model allowed decay $\mu \rightarrow e \gamma$, which proceeds through a loop process involving neutrino mixing at the unmeasureably low branching ratio below $10^{-54}$. b) A SUSY based diagram depicting smuon-selectron mixing inducing the same process.

is $\Delta L_{e}=\Delta L_{\mu}=\Delta L_{\tau}=0$, where the $L_{i}$ are assigned lepton flavor numbers. The additive sums are unchanged in any given reaction or decay. The rules 805 worked perfectly prior to the period from 1998 - 2001 when the atmospheric, solar, and reactor-based neutrino experiments demonstrated that neutrino flavors mix [66, 67, 68. These discoveries changed the rules. Neutrinos must have finite mass, and they can transform within the three generations. In neutral processes at least, lepton flavor is not a good quantum number ${ }^{13}$.

Violation of lepton flavor in the neutrino sector, in turn, implies that the decay $\mu \rightarrow e \gamma$ must occur through loop processes. However, using upper limits on the neutrino masses and the measured mixing angles, a branching ratio below $10^{-54}$ is deduced for the diagram depicted in Fig. 10 a . Needless to say, this is unmeasurably small. The bright side, is that this standard model allowed rate - which is representative of the other charged lepton flavor violation (cLFV) processes - is so tiny that any non-null measurement must indicate new physics. Indeed, calculations based on many popular SM extensions suggest large cLFV effects should appear near to current experimental limits. That is the subject of the current chapter. The richness and importance of this topic is documented 820 in numerous scholarly reviews $69,70,71,72,73$. Each generally takes on a different emphasis between theoretical expectations, experimental techniques and history, or an overall picture. Here, we highlight our view of the key topics being discussed and the three most sensitive and promising experimental programs going forward.

Comprehensive and high-sensitivity studies of tau lepton cLFV decay modes are being carried out now and further plans exist at the upgraded $B$ factory in Japan. 74]. Tagged $\tau$ decays to muons and electrons, such as $\tau \rightarrow e e e, \tau \rightarrow \mu \mu \mu$, $\tau \rightarrow e \gamma$, and $\tau \rightarrow \mu \gamma$ as well as many other combinations are being studied. Impressive sensitivity has been achieved with branching ratios typically close to

\footnotetext{
${ }^{13} \mathrm{~A}$ current hot topic in neutrino physics is the search for neutrinoless double beta decay, $0 \nu \beta \beta$. If observed, this process violates lepton number conservation and serves to prove that neutrinos are their own antiparticles-Majorana fermions. It further supports the leptogenesis explanation of why we have a baryon asymmetry in the universe.
} 

Belle-II. Additionally, the LHCb experiment has already set a competitive limit on the $\operatorname{BR}(\tau \rightarrow \mu \mu \mu)$ of $<4.6 \times 10^{-8}$, which was based on the $3 \mathrm{fb}^{-1}$ of data acquired in the first LHC run [75. Presumably this limit will be strengthened in the future as the systematics do not dominant the uncertainty.

However, it is the three low-energy muon reactions
1. $\mu^{+} \rightarrow e^{+} \gamma$
$\left(B R:<5.7 \times 10^{-13}\right)$
Ref. 8
2. $\mu^{+} \rightarrow e^{+} e^{-} e^{+}$
$\left(B R:<1.0 \times 10^{-12}\right)$
3. $\mu^{-}+N \rightarrow e^{-} N$
$\left(B R:<7 \times 10^{-13}\right)$
Ref. 9
Ref. 10

that have the more impressive limits and greater sensitivity to new physics.

840 Processes 1 and 2 represent ultra-rare decay modes. Process 3 involves the formation of a muonic atom with a nucleus - the limit quoted here is from the $\mu^{-} \mathrm{Au}$ atom-followed by coherent conversion of the muon to an electron, which is ejected with an energy close to the muon rest mass. The history of these measurement sensitivities is compiled in Fig. 11, which includes projections for experiments now being upgraded or constructed.

Note that other processes also exist. For example, the spontaneous conversion of the $M \equiv \mu^{+} e^{-}$muonium atom to anti-muonium, $\mu^{-} e^{+}$, has been searched for and a limit of $P_{M \bar{M}} \leq 8.2 \times 10^{-11}(90 \%$ C.L.) in a 0.1 T magnetic field has been set [76. Here, electron and muon number are violated in the 850 same exotic process. The physics reach is not as competitive if directly compared to current cLFV efforts and there are no current plans to improve it on the horizon [73. But, it should be noted that this is a rather complementary process that might be induced by different physics; for example, see 77 .

A relative comparison remark is in order here. Generically, for a loop-induced process, similar to those that might cause the deviation from the SM for the muon anomaly, the expected rates for processes $1: 2: 3$ scale as $389: 2.3: 1$; (here, $\mu \mathrm{Al}$ is assumed for 3) [70. The ratio implies that the present $\mu^{+} \rightarrow e^{+} \gamma$ limits greatly exceed current limits from the $3 e$ decay or the $\mu \rightarrow e$ conversion. However, the ambitious goals of modern $\mu \rightarrow e$ conversion experiments aim for 4 orders of magnitude improvements, which will close the gap.

The comparison using loop-like scaling is not the whole story, since the sensitivities vary depending on what kind of new physics leads to cLFV. Ultimately, one would like to have measured deviations in all three channels to sort out the underlying mechanism. Although there are many compelling standard model extensions, such as the SUSY process involving smuon-selectron mixing as depicted in Fig. 10b, we will follow the more generic approach developed by de Gouvêa 71. In this model-independent analysis, the cLFV processes can be compared for their respective new-physics sensitivities in terms of an effective energy scale versus a parameter that slides from loop-like exchanges to contact

interactions. de Gouvêa considers an effective Lagrangian to describe processes 


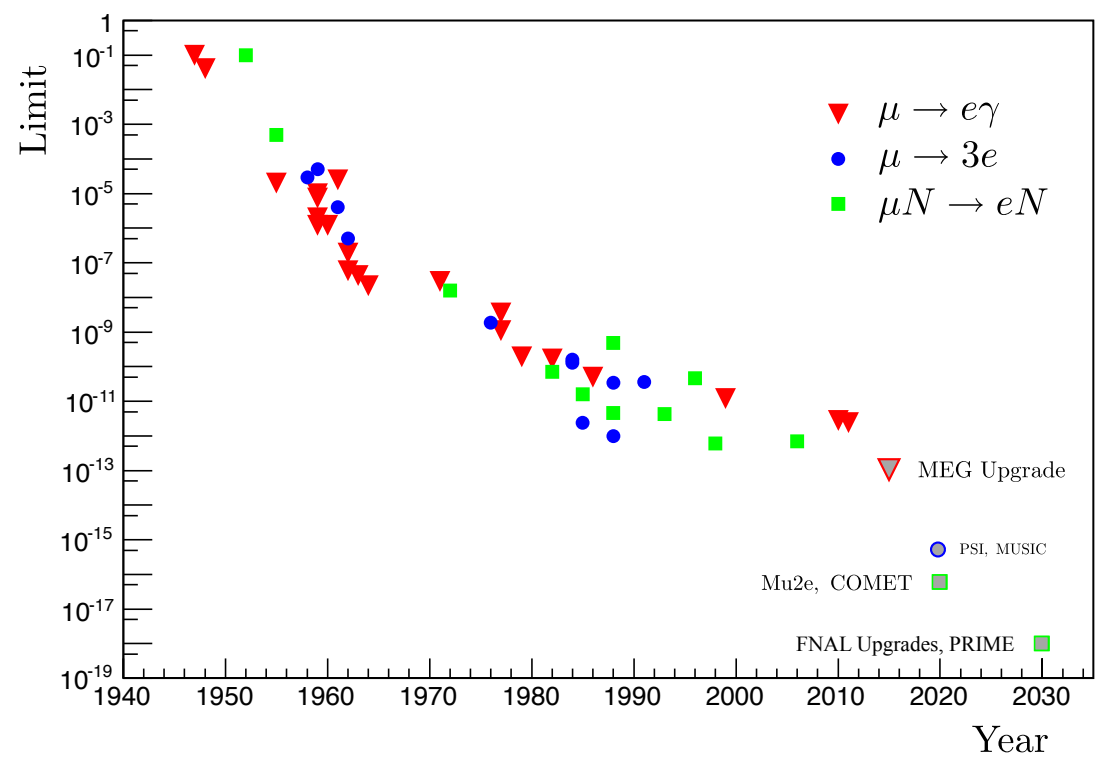

Figure 11: The solid shapes indicate the achieved $90 \%$ sensitivity limits for $\mu^{+} \rightarrow e^{+} \gamma$ (triangle), $\mu^{+} \rightarrow e^{+} e^{-} e^{+}$(circle), and the coherent conversion process $\mu^{-} N \rightarrow e^{-} N$ (square). Expected improvements from approved experimental programs have light grey interiors and the same shapes. Figure updated from 72, courtesy R. Bernstein.

(1) and (3) of the form

$$
\begin{aligned}
L_{\mathrm{cLFV}} & =\frac{M_{\mu}}{(\kappa+1) \Lambda^{2}} \bar{\mu}_{R} \sigma_{\mu \nu} e_{L} F^{\mu \nu} \\
& +\frac{\kappa}{(\kappa+1) \Lambda^{2}} \bar{\mu}_{L} \gamma_{\mu} e_{L}\left(\bar{u}_{L} \gamma^{\mu} u_{L}+\bar{d}_{L} \gamma^{\mu} d_{L}\right) .
\end{aligned}
$$

Here $L$ and $R$ are fermion field chiralities and $F^{\mu \nu}$ is the photon field. If the dimensionless parameter $\kappa \ll 1$ the process is dominated by a magnetic moment type operator (as above). In contrast, if $\kappa \gg 1$, the four-fermion operators (2nd line) dominate, representing a point-like contact interaction. The parameter $\Lambda$ is an effective energy scale that extends for current experimental sensitivity goals to 1000's of TeV - for optimized coupling - well in excess of any collider reach. This comparison is plotted in the left panel of Fig. 12 for both current experimental limits and the future projects described below. The sensitivity 880 from $\mu^{+} \rightarrow e^{+} \gamma$ searches falls off quickly for contact-like interactions, unlike $\mu-e$ conversion, which rises.

A similar Lagrangian (the second term is different) can be used to describe 
reactions (1) and (2):

$$
\begin{aligned}
L_{\mathrm{cLFV}} & =\frac{M_{\mu}}{(\kappa+1) \Lambda^{2}} \bar{\mu}_{R} \sigma_{\mu \nu} e_{L} F^{\mu \nu} \\
& +\frac{\kappa}{(\kappa+1) \Lambda^{2}} \bar{\mu}_{L} \gamma_{\mu} e_{L}\left(\bar{e}_{L} \gamma^{\mu} e\right),
\end{aligned}
$$

where $\kappa$ and $\Lambda$ correspond to similar definitions. The right panel of Fig. 12 plots 885 these sensitivities. The $\mu \rightarrow$ eee process will be several orders of magnitude less sensitive compared to $\mu^{+} \rightarrow e^{+} \gamma$ for loop-like processes; however, for contacttype interactions there is a part of the new-physics search space that favors the 3e decay mode.

An important word of caution is in order here so as not to over-interpret the vertical axis in Fig. 12. The new physics "mass scale" $\Lambda_{\text {cLFV }}$ is not to be interpreted strictly as mass. It is product of mass and flavor-violation mixing, where the latter is similar to the mixing angles in a CKM or PMNS matrix for quarks and neutrinos. The mixing can be large - neutrino like - or very smallquark like, or it can be anything at all. With a cLFV experiment there is but one-measurement and two parameters, which emphasizes the need for multiple measurements (channels) and comparisons to other precision measurements. For example, consider the situation where the new physics impacts both $g-2$ (flavor conserving) and cLFV. Then the new physics scale can be related by $\Theta_{e \mu} \Lambda_{\mathrm{cLFV}}^{2}=\Lambda_{g-2}^{2}$, where $\Theta_{e \mu}$ represents the level of flavor-mixing in the new$\Theta_{e \mu} \ll 1$ implies small mixing [71].

\subsection{Experimental challenge of muon cLFV}

The goals for modern charged lepton flavor violation experiments all aim at single event branching ratios of a few times $10^{-14}$ to $10^{-17}$ range. To be sensitive to such stunningly rare processes requires an event signature that is unique with respect to backgrounds, and exceptional design and execution by the experimental team. Because all processes involve muons at rest, the energy scale of the emitted particles is low-below $m_{\mu}$ for the decay studies and equal to $\sim m_{\mu}$ for the conversion process. From a detector design perspective, this is a challenge, requiring ultra-thin tracking detectors and especially high-resolution calorimetry. The sub-dominant, but ordinary, muon decay modes are problematic. Radiative muon decay, $\mu^{+} \rightarrow e^{+} \nu_{e} \bar{\nu}_{\mu} \gamma$, occurs with a branching ratio of $1.4(4) \%$ and radiative decay with internal conversion, $\mu^{+} \rightarrow e^{+} e^{-} e^{+} \nu_{e} \bar{\nu}_{\mu}$, occurs at the $(3.4 \pm 0.4) \times 10^{-5}$ level. For the corners of phase space where the neutrinos escape with very little energy, these decay modes can mimic the cLFV processes (1) and (2), at a many orders of magnitude greater rate.

To achieve sensitivities below $10^{-14}$ a considerable number of stopped muons is required (well above $10^{14}$ after factoring in efficiencies). In context, the MuLan lifetime experiment described in Sec. 2.2.1 measured $\tau_{\mu}$ to $1 \mathrm{ppm}$ using $2 \times$

${ }_{920} 10^{12}$ decays, and utilizing a high-intensity beamline at PSI. The cLFV program requires, in contrast, muon samples larger by factors of $10^{3}-10^{5}$. The highestintensity $\pi E 5$ beamline at PSI can deliver a dc rate of $10^{8}$ muons/sec, which 

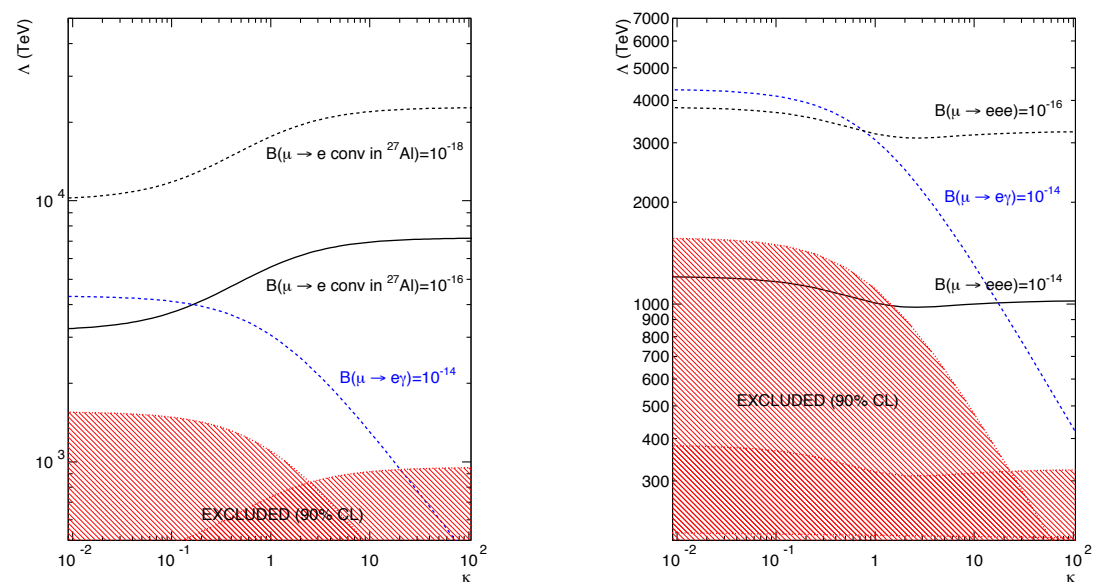

Figure 12: Comparison of the new physics mass scale reach for the cLFV experiments using current achieved sensitivities and goals of planned programs. In both panels, $\kappa \ll 1$ corresponds to loop-like processes, transitioning to 4 -fermion contact interactions for large values of $\kappa$. Left panel: $\mu^{+} \rightarrow e^{+} \gamma$ versus $\mu^{-} N \rightarrow e^{-} N$ coherent conversion experiments. Right panel: $\mu^{+} \rightarrow e^{+} \gamma$ versus $\mu^{+} \rightarrow e^{+} e^{-} e^{+}$. Figure updated from [71, courtesy A.de Gouvêa.

is 10 times higher than MuLan used. The dc nature of the beam structure is ideal for the decay programs that observe one event at a time. However, rates, multiple events are in the detector at o coincident timing and position resolution to distinguish individual decays. To reach the ultimate goal of the $3 e$ experiment of $10^{-16}$ will require a new ultrahigh intensity beamline having $10 \times$ greater flux than presently exists. For the next-generation $\mu \rightarrow e$ conversion experiments - the goals there approach the $10^{-17}$ level - an entirely different concept is required, which we describe below.

\subsubsection{Measuring $\mu^{+} \rightarrow e^{+} \gamma$}

The decay mode $\mu^{+} \rightarrow e^{+} \gamma$ is being pursued by the MEG collaboration at PSI. Their results to date set the limit $B R_{e \gamma}<5.7 \times 10^{-13} ; 90 \%$ C.L. [8]. The decay mode features the back-to-back emission of a positron and a gamma ray, each having energies equal to $m_{\mu} / 2 \approx 53 \mathrm{MeV}$. Positive muons at a dc rate of up to $30 \mathrm{MHz}$ are stopped in a thin polyethylene disk centered in a cylindrical geometry. A tracking drift chamber system and a high-resolution liquid xenon (LXe) calorimeter are key components, see Fig. 13.

A unique feature of the experiment is the COnstant Bending RAdius (COBRA) magnet. Its gradient field shape is designed to maintain a near-constant bending radius of decay positrons, independent of their initial pitch angle, and further, to sweep away the decay trajectories to the upstream or downstream side of the stopping target. The unique calorimeter uses 862 PMTs, submerged in LXe and mounted on all surfaces to sum the light and to therefore provide energy, time-of-arrival, and location of the interaction of the gamma in the xenon 

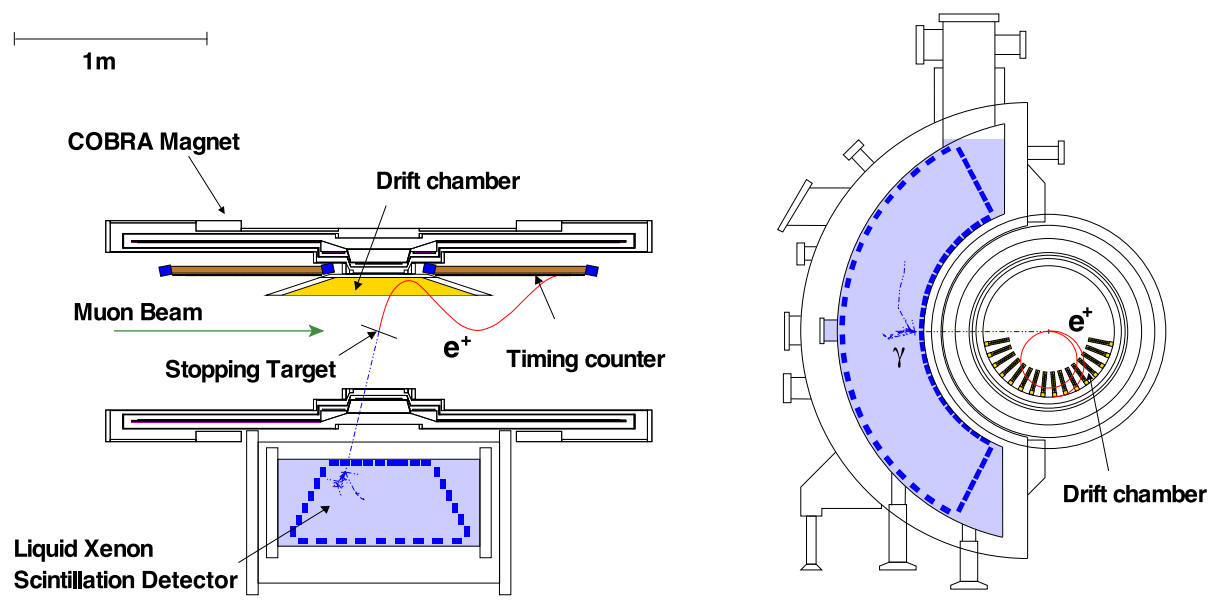

Figure 13: The side and front schematic of the MEG detector with a hypothetical $\mu \rightarrow e \gamma$ event superimposed. Figure courtesy MEG collaboration.

bath. The ultra-low-mass drift chamber sits in a half circle below the target. A scintillator array is used to precisely establish the timing needed. At signal energies $E_{\gamma}=E_{e^{+}}=m_{\mu} / 2$, the tracker resolution is $1.5 \%$ and the calorimeter resolution is $4.5 \%$. The overall acceptance for decays of interest is $\sim 18 \%$. [78]

Two types of backgrounds enter, those from radiative decay, and those from pileup events. The key to distinguish these from signal are the performance parameters of the detectors. We reproduce Fig. 14 from [8] because it perfectly illustrates the case by displaying the allowed signal event regions, which are dictated by detector resolutions. If the resolutions can be improved, the signal region can be reduced, increasing the overall sensitivity. The right panel shows the $\cos \Theta_{e \gamma}$ vs. $\Delta t$ plane, where $\Theta_{e \gamma}$ is the difference between the emitted angles of the electron and gamma, and $t_{e \gamma}$ is the difference in their timing. The left panel shows the $e^{+}-\gamma$ energy plane with the enclosed ellipses defining the signal region. Improvements in energy-, angle-, and timing-resolution of the involved detectors allows one to shrink the good-event windows proportionally.

The MEG II approved upgrade program [79] is designed to handle muon stopping rates on a thinner target at rates up to $70 \mathrm{MHz}$; the final statistics will require three years of running, beginning in 2016. Resolutions will be improved by about a factor of 2 on all detectors. New UV-sensitive SiPMs will replace the 965 PMTs and provide more uniform light collection. A single-volume drift chamber system will replace the current vane structures. The positron scintillator counters provide $30 \mathrm{ps}$ timing resolution. The overall goal for the branching ratio is $<4 \times 10^{-14}$.

\subsubsection{Measuring $\mu^{+} \rightarrow e^{+} e^{-} e^{+}$}

The unique signature of the $\mu^{+} \rightarrow e^{+} e^{-} e^{+}$reaction is the three coincident $e^{ \pm} \mathrm{S}$ emanating from a common vertex and carrying, in sum, a total energy cor- 

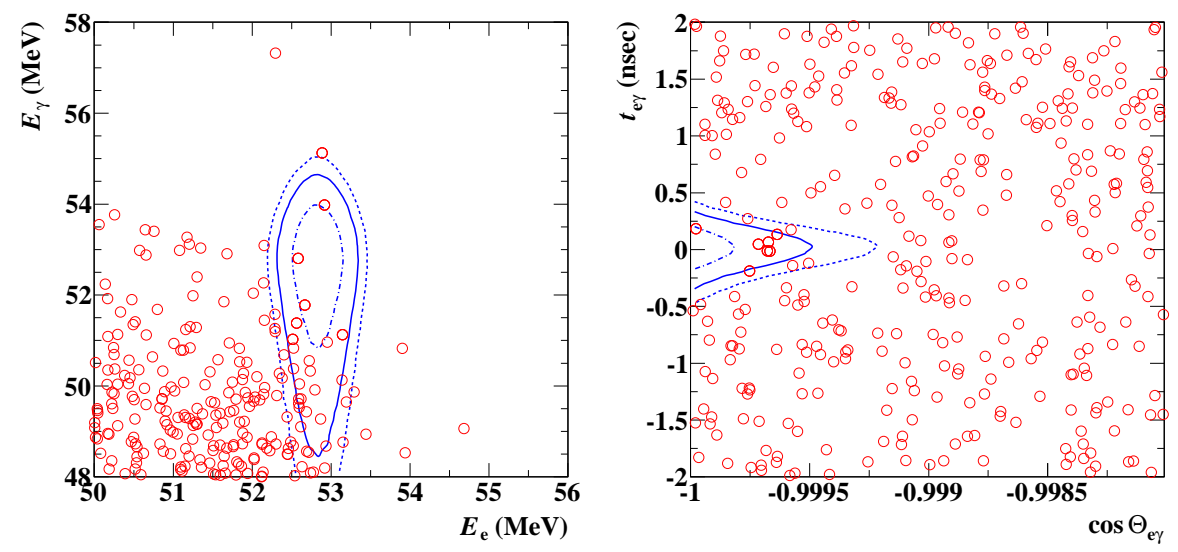

Figure 14: The event search regions from the MEG publication 8], which illustrates the importance of a comprehensive approach to improving detector resolutions and timing in order to narrow the allowed good event region. The left panel shows the $E_{\gamma}-E_{e}$ plane, where the allowed region is centered at $m_{\mu} / 2$ in both projections. The right panel is the $\Delta t-\cos \Theta_{e \gamma}$ plane where the region of interest picks out simultaneous, back-to-back decay products. The blue enclosed regions are signal probability distribution function contours of $1-, 1.64-$, and $2 \sigma$. Figures courtesy MEG collaboration, Ref. [8]

responding to the full muon mass, and a net momentum of zero. This measurement is a particular challenge, as the three tracks must be accurately measured with very high resolution for each event, and the triple coincidence implies that 975 the tracker efficiency be very high. Candidate three $e^{ \pm}$events having a total energy near to the muon mass must be considered. Background processes such as the internal-conversion decay, generally yield lower event energy sums and distort the momentum balance such that the event should be outside the search window. Multiple scattering and energy loss in the tracking chambers could distort the interpreted energy if the detector design does not factor that in properly. Of the three processes discussed, this one has been idle for a long time see [9] with $B R_{3 e}<10^{-12}$, but the new Mu3e collaboration at PSI is seeking to study it in stages. They are approved with a goal of $10^{-15}$ using existing beamlines. A final phase is imagined but requires a muon beam with a 985 rate of $10^{9} \mathrm{~Hz}$, which is a non-trivial development and investment 80 .

The key to the ultimate precision is an extremely high muon stopping rate distributed over a double cone thin aluminum target. The decay trajectories are then imaged precisely using state-of-the-art thin silicon pixel detectors assembled into cylindrical geometries. Timing scintillators between tracking layers mark the event times. To achieve the ultimate sensitivity of $10^{-16}$, the resolution on the reconstructed muon mass must be roughly $0.5 \mathrm{MeV}$ (the mass of a single electron!), just to suppress the internal conversion look-a-like decay mode $\mu^{+} \rightarrow e^{+} e^{-} e^{+} \nu_{e} \bar{\nu}_{\mu}$. This further assumes excellent sub-ns coincident timing of the tracks, and does not factor in accidental backgrounds.

A schematic diagram of the approved Mu3e experiment is shown in Fig. 15 


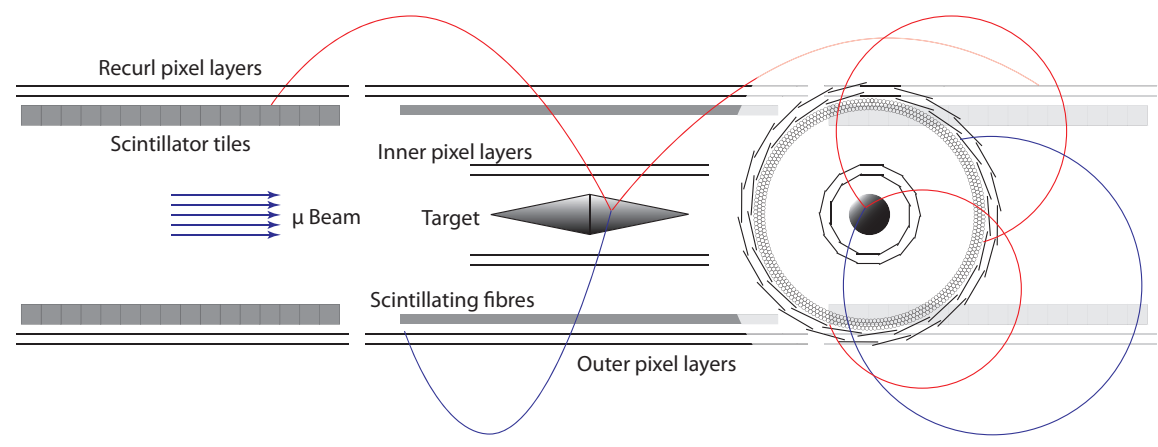

Figure 15: Side and end views of the proposed Mu3e detector with a single simulated $\mu^{+} \rightarrow e^{+} e^{-} e^{+}$event displayed. The conical target distributes the high rate of muon stops in different locations forming distinct vertices in the reconstruction of tracks. The ultrathin pixelated silicon tracker extends up- and down-stream. The scintillating fiber hodoscope provides precision timing. The experiment will be assembled and run in phases; the approved Phase IB configuration is shown. Figure courtesy Mu3e collaboration.

In the first phase, only the central pixel detector surrounding the target will be used and the beam rate in the $\pi E 5$ line will be limited to $10^{7} \mu^{+} / \mathrm{s}$. The physics goal is a BR sensitivity of roughly $10^{-14}$. The next step increases the tracking volume, adds scintillating-fiber trackers and other components, and increases the muon stopping rate to $10^{8} \mathrm{~Hz}$. The aim is a sensitivity of $10^{-15}$.

\subsubsection{Measuring $\mu^{-} N \rightarrow e^{-} N$ coherent conversion}

The quantity of interest in a $\mu \rightarrow e$ experiment is the ratio

$$
R_{\mu e}=\frac{\Gamma\left[\mu^{-}+A(Z, N) \rightarrow e^{-}+A(Z, N)\right]}{\Gamma\left[\mu^{-}+A(Z, N) \rightarrow \nu_{\mu}+A(Z-1, N)\right]}
$$

where the rate of ordinary capture in the denominator is already quite well known. For example, for typical stopping targets such as aluminum, which we will continue to assume below, one can observe that the muon lifetime of a $\mu-\mathrm{Al}$ atom is reduced to $\approx 864 \mathrm{~ns}$. From

$$
\frac{1}{\tau_{\mu A l}}=\Gamma_{\text {tot }}=\Gamma_{\text {decay }}+\Gamma_{\text {capture }}
$$

and knowing the free lifetime, one obtains the capture rate $\Gamma_{\mu A l} \approx 7 \times 10^{5} \mathrm{~s}^{-1}$. The numerator in Eq. 16, on the other hand, takes on a new level of challenge.

While the history of $\mu \rightarrow e$ experiments is well covered in Ref. 72, here we concentrate on the two major effort $\$ 4$ that ambitiously aim at single-event sensi-

${ }^{14}$ We note that the less ambitious DeeMe 81] experiment at the J-PARC Material Life 
tivities approaching $\approx 3 \times 10^{-17}$, a 4 order of magnitude improvement compared to present limits. The two projects - Mu2e [82] at Fermilab and COMET [83] at J-PARC - share many similar conceptual design features, while individual technical solutions differ. We discuss the generic method here using some examples for clarity.

A principle requirement is a sample of $\sim 6 \times 10^{17}$ muonic atoms. No existing secondary decay beamline can provide such a flux to accumulate that many events in a few years of running. Furthermore, the muons should be delivered with a beam-on / beam-off time structure commensurate with the 1015 muon lifetime in the target material. Ideally, bursts of muons should arrive and stop in a suitable nuclear target during an accumulation period lasting some 100 's of ns at most, followed by a beam-off and background-free quiet measuring period. During that time, the detector is sensitive to the unique $\mu \rightarrow e$ signature event having a mono-energetic electron emitted with $E_{e}=105 \mathrm{MeV}$; the value represents the muon mass, less the atomic binding energy. For maximally efficient data taking, the combined accumulation-measuring cycle should be $\mathcal{O}\left(2 \tau_{(\mu A l)}\right) \approx 1.7 \mu$ s and repeat as efficiently and continuously as possible, subject to the main accelerator macro cycle timing.

To provide some numbers as an example, the Mu2e statistical budget as1025 sumes an average data-taking rate of $\sim 10^{10}$ formed muonic atoms per second and $6 \times 10^{7} \mathrm{~s}$ of running time. Using the reduced fraction of the Fermilab $1.33 \mathrm{~s}$ accelerator cycle available, they must collect nearly 60,000 atoms in a $1.7 \mu \mathrm{s}$ cycle. How is this possible?

Both Mu2e and COMET follow a recipe credited to Lobashev and proposed 1030 for MELC in 1989 [84]. A pulsed proton beam is slammed through a target that is enveloped in a superconducting "production" solenoid. The produced pions are contained by the magnetic field, as are their decay muons below a momentum of $\approx 40 \mathrm{MeV} / c$. The solenoid field has a strong gradient to direct the spiraling secondaries out along the upstream direction into a curved "transport" solenoid.

1035 This either $S-(\mathrm{Mu} 2 \mathrm{e})$ or $U-(\mathrm{COMET})$ shaped device provides momentum and sign selection of the captured muons and rejects by line-of-sight the transport of neutral particles from the production target. It is long enough to allow most of the pions to decay before emerging at the exit. The final stage is yet another solenoid - the "detector" region-where the muons will first stop in thin $\mathrm{Al}$

1040 blades. In the same volume, and downstream further, a specialized spectrometer system is positioned to intercept the highest energy decay electrons (well above the Michel endpoint). Figure 16 illustrates the geometry and scale of the Mu2e interpretation of this concept. As noted, the transport section for COMET is $U$ shaped,and the detector section is also $U$ shaped, the bend between target and tracker.

Assuming now that one has a sample of formed $\mu \mathrm{Al}$ atoms, with the muon

Science Facility aims to turn on sooner than these efforts, with the single event sensitivity of $2 \times 10^{-14}$. If achieved, that level will be more than an order of magnitude improvement compared to current limits. 


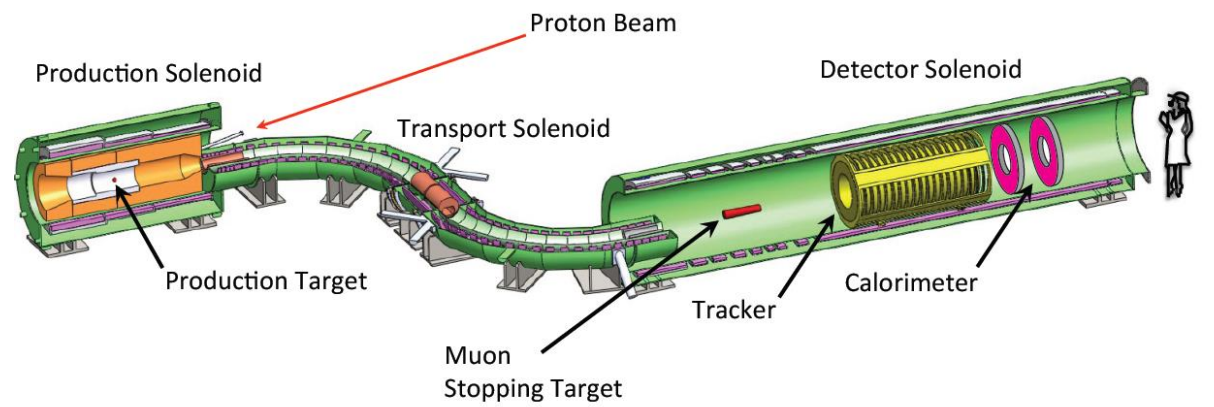

Figure 16: The Mu2e Experimental concept. The pulsed proton beam enters the production solenoid and passes through a tungsten rod. Pions produced there, decay to muons which are directed via magnetic gradients into the $S$-shaped transport solenoid, arriving in the detector solenoid region. The momentum-selected negative muons stop in an $\mathrm{Al}$ target. The signature decay $105 \mathrm{MeV}$ electron is measured with a tracking chamber followed by an electromagnetic calorimeter. Figure courtesy Mu2e collaboration.

having cascaded to the $1 \mathrm{~S}$ ground state, the next critical issue is associating a high-energy emerging electron with $\mu \rightarrow e$ conversion. One might naively assume this is quite far away from the Michel endpoint $E_{e}=m_{\mu / 2}$, which it is; however, that only applies for an unbound muon. The muon in a $\mu \mathrm{Al}$ atom can "decay in orbit" (DIO) about $39 \%$ of the time. The corresponding kinematics of the in-motion system allows - in principle - the emitted electron to carry away the entire muon mass in energy, with the nucleus participating to conserve momentum. This is a well-known effect and the probability of an electron close to the $105 \mathrm{MeV}$ signal region is indeed quite rare (or else, conversion experiments would have long ago ceased). With the approaching efforts of Mu2e and COMET, the exactness of the DIO spectrum had to be revisited to determine the real sensitivity of these major new efforts. In a recent calculation, Czarnecki, Tormo and Marciano included proper nuclear recoil effects and generated the expected spectra of electron rate vs. energy shown in Fig. 17. The left figure provides the full range of energies; the right panel is a blowup of the critical region from 100 to $105 \mathrm{MeV}$. The scale is in $1 \mathrm{MeV}$ bins of the relative sensitivity units projected by the experimentalist. It is clear that very high resolution is thus required above $100 \mathrm{MeV}$ in order to

1065 keep the DIO fraction below the single event sensitivity. At the time of this writing, both Mu2e and COMET are in construction phases.

\section{Muon Dipole Moments}

\subsection{Terminology for muon magnetic and electric dipole moments}

The muon's magnetic dipole moment (MDM) $\vec{\mu}_{\mu}$ is related to its intrinsic spin $\vec{s}$ with a proportionality that includes the $g$-factor, which is embedded in 

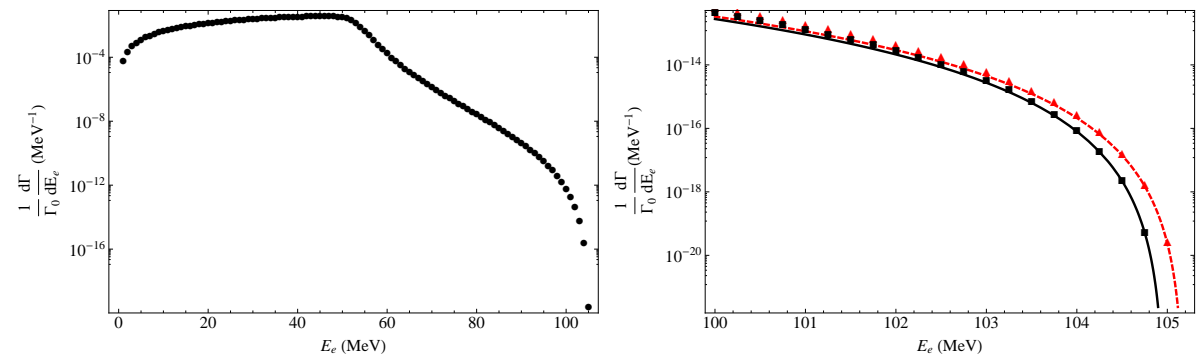

Figure 17: Left: Decay in orbit electron energy spectrum assuming an aluminum target for the $\mu \mathrm{Al}$ atom. Right: Blowup of the critical signal region from 100 to $105 \mathrm{MeV}$ where the black squares include recoil effects; red triangles do not. Figures courtesy A. Czarnecki, Ref. 85]

the relation

$$
\vec{\mu}_{\mu}=g_{\mu}\left(\frac{q}{2 m}\right) \vec{s}
$$

The provision for a possible nonzero electric dipole moment (EDM) $\vec{d}_{\mu}$, with a magnitude parameterized by $\eta$, is given by

$$
\vec{d}_{\mu}=\eta_{\mu}\left(\frac{q \hbar}{2 m c}\right) \vec{s}
$$

Notice that any EDM must be aligned along the angular momentum axis, the only vector in the system. We first discuss the important topic of the MDM, where the measured value is in disagreement with the standard model (SM) prediction. The measured EDM is compatible with zero, but it is not yet determined to high enough precision to challenge SM completeness. The EDM prospects are discussed in Sec. 5.4 .

\subsection{Magnetic dipole moment}

The Dirac equation predicts $g_{\mu} \equiv 2$ for the structureless, spin- $1 / 2$ muon. Radiative corrections from electromagnetic (QED), weak and hadronic loops give rise to a so-called anomalous magnetic moment $a_{\mu}$; that is, $g_{\mu}=2\left(1+a_{\mu}\right)$, or more commonly stated: $a_{\mu} \equiv(g-2) / 2$. The anomaly is a small correction, with $a_{\mu} \approx 1 / 850$. Figure 18 illustrates example Feynman diagrams that must be evaluated to arrive at the SM value for $a_{\mu}$. In practice, more than 10,000 of these topologies have been calculated. These include QED through 5 loops $\left(10^{t h}\right.$ order), weak exchanges through 3 loops, leading-order hadronic vacuum polarization (Had-LO) — which is determined from experimental data-

1085 and higher-order hadronic light-by-light scattering (HLbL), which is difficult to evaluate owing to the non-perturbative nature of QCD at low energies. An excellent and detailed summary can be found in the textbook and review by Jegerlehner [86, 87. Figure 19 displays the magnitudes and uncertainties of the SM contributions along with the current summary. 
a)

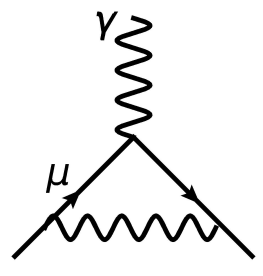

b)

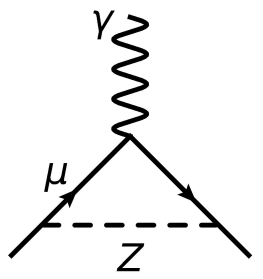

c)

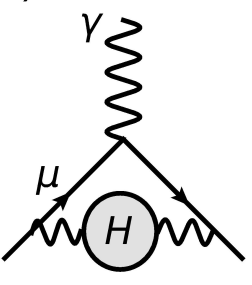

d)

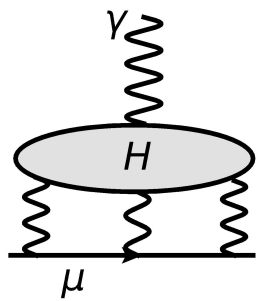

Figure 18: Example diagrams that contribute to the muon anomalous magnetic moment. a) Leading-order QED "Schwinger" term; b) Electroweak $Z$ exchange diagram; c) Lowest-order hadronic vacuum polarization; d) Hadronic light-by-light scattering.

The experimental history of $(g-2)$ measurements is quite rich and well reviewed; see, for example Ref. [88. A series of storage ring experiments was conducted at CERN in the 1960's and 70's and the highest precision effort was completed at Brookhaven National Laboratory in 2001. As this review will describe, next-generation efforts are being prepared [89, 90. The vertical black bars in Figure 19 indicate the achieved uncertainties of the three most recent experiments (solid lines) and the precision goal (dashed line) of the new Fermilab experiment.

Both experiment and theory are now known to similar sub-ppm uncertainty and the comparison provides a sensitive test of the completeness of the standard model. If the SM accounting is accurate, and the experimental result is correct, the present comparison already begins to suggest the existence of some new physics process that affects $a_{\mu}$.

Currently accepted SM predictions for $a_{\mu}(\mathrm{SM})$ are

$$
\begin{aligned}
& a_{\mu}\left(\mathrm{SM}_{\mathrm{a}}\right)=116591802(49) \times 10^{-11} \quad(0.42 \mathrm{ppm}) \quad \text { and } \\
& a_{\mu}\left(\mathrm{SM}_{\mathrm{b}}\right)=116591828(50) \times 10^{-11}(0.43 \mathrm{ppm}) .
\end{aligned}
$$

The subscripts a ([91]) and b (92]) represent slightly different evaluations of the leading-order hadronic vacuum polarization contributions. In both cases a theoretically sound dispersion relationship is used, which evaluates $a_{\mu}$ from an appropriately weighted integral of $e^{+} e^{-} \rightarrow$ hadrons absolute cross section data, summed over all energies. The uncertainty here is largely experimental. The HLbL contribution, on the other hand, is model based and its quoted theoretical uncertainty is only estimated at present.

The experimental $a_{\mu}$ is based solely on the BNL E821 measurement [],

$$
a_{\mu}(\operatorname{Exp})=116592091(63) \times 10^{-11} \quad(0.54 \mathrm{ppm}) .
$$

The difference between experiment and theory - the " $(g-2)$ test" - is

$$
\begin{aligned}
\Delta a_{\mu}\left(\operatorname{Exp}-\mathrm{SM}_{\mathrm{a}}\right) & =289(80) \times 10^{-11}(3.6 \sigma) \quad \text { or } \\
\Delta a_{\mu}\left(\operatorname{Exp}-\mathrm{SM}_{\mathrm{b}}\right) & =263(80) \times 10^{-11}(3.3 \sigma) .
\end{aligned}
$$




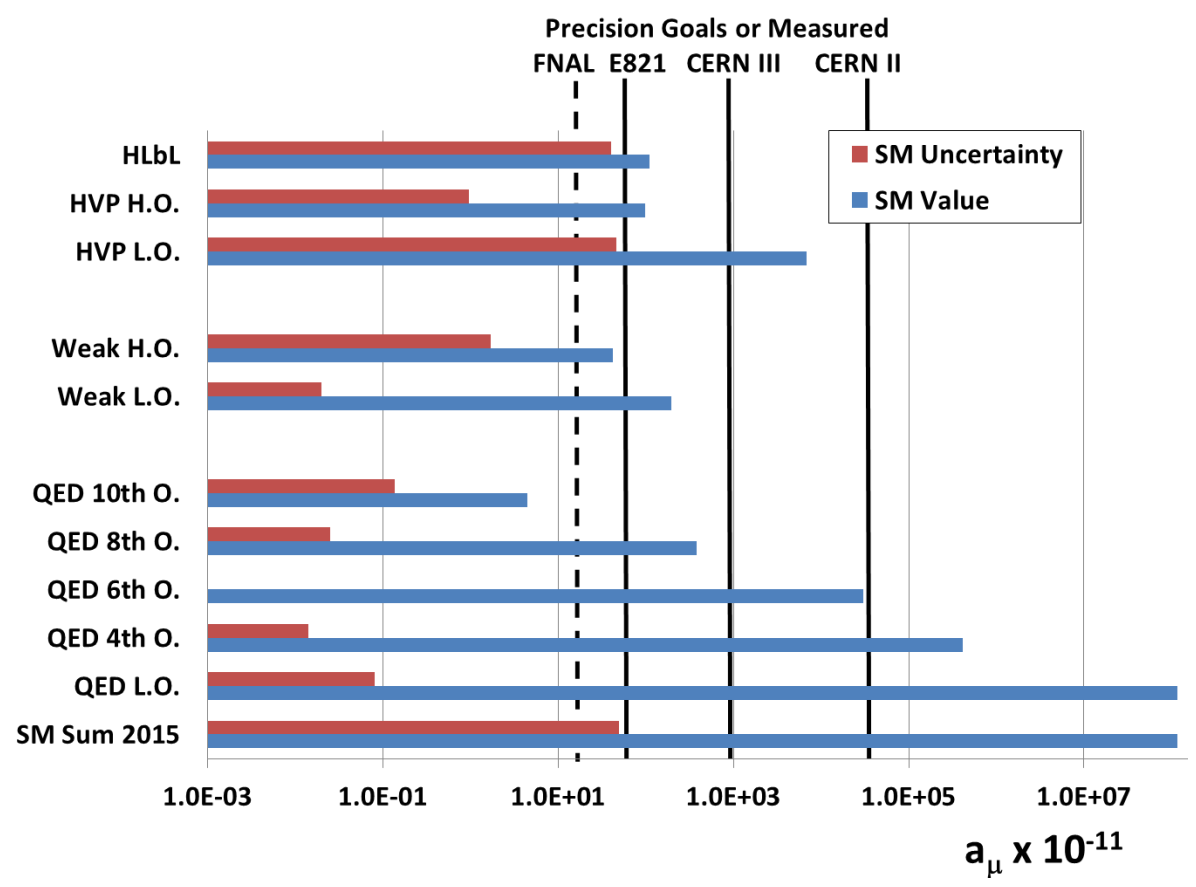

Figure 19: The horizontal bars display the magnitudes of the standard model contributions (blue) and their present uncertainties (red). Terms include QED through $10^{\text {th }}$ order, leadingand higher-order weak, leading- and higher-order hadronic vacuum polarization, and hadronic light-by-light. The 2014 summary is given along with its uncertainty of $49 \times 10^{-11}$. The solid vertical black lines are placed at the achieved final precisions of the CERN II (27-ppm), CERN III (7.3 ppm), and BNL E821 experiments (0.54 ppm). The dashed black line illustrates the precision goal of FNAL E989 (0.14 ppm) 
In both cases, the result is quite provocative. The magnitude of the difference is large - it exceeds the electroweak contributions - and the statistical significance is large. This persistent discrepancy has led to many speculations of new physics scenarios and to challenges to the accounting procedures, data selection, and hadronic models used to determine the SM expectation. The resolution-new physics or some type of theory or experimental error - has led to the launching of two major new experimental thrusts, with one of them that aims to reduce $\delta a_{\mu}(\exp )$ fourfold to an absolute uncertainty of $16 \times 10^{-11}$. In parallel, a vigorous 1120 theory campaign is taking place [93, 94].

As Fig. 19 illustrates, the QED and weak uncertainties are already well below any experimental reach. Improvements by a factor of 2 in the dominant Had-LO uncertainty are expected from new data sets at VEPP-2000 (Novosibirsk) and BESIII (Beijing), along with continued analyses of BaBar and Belle existing data sets 93.

While the magnitude of the complete HLbL contribution is $\sim 110 \times 10^{-11}$, the quoted uncertainties can be as large as $35 \%$. A recent review of the status of the HLbL contributions is given in 95]. Initiatives going forward include those based on lattice QCD [96, and new $\gamma^{*}$ physics measurement programs 1130 at BESIII and KLOE (Frascati) that aim to build a data-driven approach to leading HLbL terms 93 .

\subsubsection{New physics possibilities from $(g-2)$}

What is nature trying to tell us if the current discrepancy $\Delta a_{\mu}$ remains as large as it is and the significance eventually exceeds $5 \sigma$ ? While numerous explanations exist in the literature, it is instructive to take a more generic approach here as first outlined by Czarnecki and Marciano [97, and elaborated on by Stockinger [98. The magnetic moment is a flavor- and CP-conserving, chirality-flipping, and loop-induced quantity. Any new physics (N.P.) contributions will typically contribute to $a_{\mu}$ as

$$
\delta a_{\mu}(N . P .)=\mathcal{O}[C(N . P .)] \times \frac{m_{\mu}^{2}}{M^{2}},
$$

where $M$ is a new physics mass scale and $C(N . P$.) is the model's coupling strength, which is then also common to the same new physics contributions to the muon mass. That means

$$
C(N . P .) \equiv \frac{\delta m_{\mu}(N . P .)}{m_{\mu}} .
$$

Different predictions for $a_{\mu}($ N.P.) are illustrated in Fig. 20 for various coupling strengths, $C\left(N . P\right.$.) versus the present and future $\delta a_{\mu}$ limits, the latter being the combined uncertainty from SM theory and experimental sensitivity. For radiative muon mass generation, $C(N . P)=.\mathcal{O}(1)$, which implies $a_{\mu}$ probes the multi-TeV scale. For models with typical weak interaction coupling, $C(N . P)=.\mathcal{O}(\alpha / 4 \pi)$, the implied mass scale is very light, arguably ruled out by direct measurements. In contrast, models with enhanced coupling such as 
supersymmetry, unparticles and extra dimensions are represented by the central band, where the overlap with $a_{\mu}$ corresponds to the $\mathrm{TeV}$-scale physics regime. Focussing on SUSY, the expected contribution to $a_{\mu}$ has the following behavior:

$$
a_{\mu}^{\mathrm{SUSY}} \approx 130 \times 10^{-11}\left(\frac{100 \mathrm{GeV}}{M_{\mathrm{SUSY}}}\right)^{2} \tan \beta \operatorname{sign}(\mu) .
$$

Here, $\tan \beta$ is the ratio of vacuum expectation values for the two Higgs doublets and $\operatorname{sign}(\mu)$ is the sign of the higgsino mass parameter, taken to be +1 from current $(g-2)$ constraints. A wide, yet natural, range for $\tan \beta$ of $5-50$ provides the width of the central band. It is clear from this figure that the LHC reach overlaps well with the simplest SUSY expectations, which have been highly motivated given the compatibility with the current $(g-2)$ result [99]. However, the lack of any signal in the $7-8 \mathrm{TeV}$ data taking at the LHC has pushed the mass scale near to and even above $1 \mathrm{TeV}$, which has also spawned many variants on the simplest SUSY models that must remain compatible with a wide suite of experimental limits and the $(g-2)$ "signal;" see reviews: [100, 101].

Hypothetical dark photons are very weakly interacting and very light particles that could produce a large enough contribution to $a_{\mu}$ to explain the discrep-

${ }_{1145}$ ancy between experiment and theory [102. In Fig. 20 a dark photon mechanism would correspond to a new band to the left of the red band that crosses the $\Delta a_{\mu}$ region in the $10-100 \mathrm{MeV}$ mass range. The coupling strength would be appropriately tuned and small. While initially suggestive of a neat explanation for $(g-2)$ recent experiments [103] have all but ruled out the simplest versions of the theory and its implied parameter space; however, more complex scenarios - ones that imply a dark $Z^{\prime}$ for instance - remain viable [104.

\subsection{Experimental considerations}

Given the high impact of the $(g-2)$ experiments, numerous general and detailed reviews have been written [106, 107, 108, along with a comprehensive publication by the E821 collaboration [6]. Here we will describe the essential features that enable such a precision measurement. The persistent $>3 \sigma$ discrepancy between experimental and theoretical $a_{\mu}$ over the past 10 years has led to the development of two new experiments that are being designed to resolve the situation. FNAL E989 [89] and J-PARC E34 [90] are following different ap-

1160 proaches. In both cases, intense bunches of polarized muons are injected into a uniform-field magnet, where the muon spin precession frequency is proportional to $a_{\mu}$. The decay positrons are measured as a function of time, the rate of which features a sinusoidal modulation imprinted on the exponential decay. Beyond this, the difference in implementation is quite significant. We first outline common considerations for a $(g-2)$ experiment and follow with brief descriptions of the two new experimental campaigns.

The spin of a muon at rest will precess in a magnetic field $\vec{B}$ at the Larmor frequency, $\vec{\omega}_{L}=-g q \vec{B} / 2 m_{\mu}$. A precision measurement of $\omega_{L}$ together with an equally precise determination of $\vec{B}$ gives $g$. Since $(g-2)$ is known to sub-ppm already, a direct measurement of $g$ would need to be at the sub-ppb level to be 

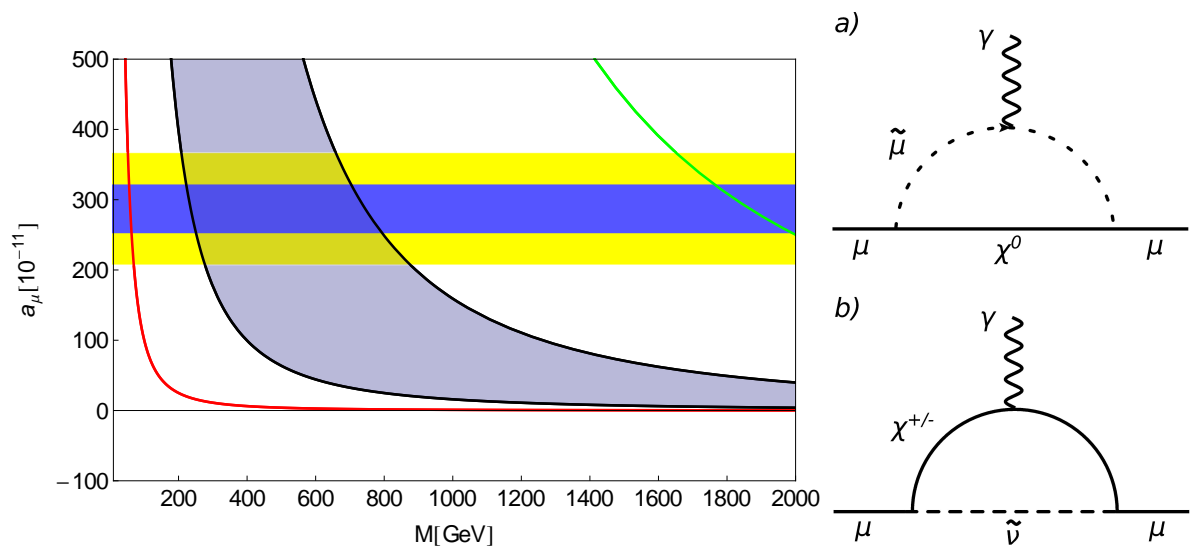

Figure 20: Left: Generic classification of mass scales vs. $a_{\mu}$ contributions from new physics sources. Green line: radiative muon mass generation; Red line: $Z^{\prime}, W^{\prime}$, universal extra dimensions, or Littlest Higgs models with typical weak-interaction scale coupling; Purple band: unparticles, various extra dimension models, or SUSY models where the coupling is enhanced. The width illustrates a $\tan \beta$ range of $5-50$ for SUSY models. The yellow horizontal band corresponds to the current difference between experiment and theory and the blue band is an improvement with a combined theory and experimental error of $34 \times 10^{-11}$. Figure courtesy D. Stockinger; see also [105]. Right: Neutralino-smuon (a) and Chargino-sneutrino (b) one-loop SUSY contributions to $a_{\mu}$ 99.

competitive. However, the muon mass $m_{\mu}$ is "only" known to $34 \mathrm{ppb}$, so that quantity too would need a major improvement.

In contrast, a measurement using in-flight muons in a magnet can directly determine $(g-2)$. It is based on the difference between the cyclotron and spinprecession frequencies for a polarized ensemble of muons that circulates in the horizontal plane of a uniform vertical magnetic field. The cyclotron frequency when $\vec{B} \cdot \vec{P}_{\mu}=0$ is

$$
\vec{\omega}_{c}=-\frac{q \vec{B}}{m \gamma}
$$

and the spin turns at frequency

$$
\vec{\omega}_{s}=-\frac{g q \vec{B}}{2 m}-(1-\gamma) \frac{q \vec{B}}{\gamma m}
$$

owing to the torque on the magnetic moment and including the Thomas precession effect for the rotating reference frame [109.

If $g$ were exactly equal to $2, \vec{\omega}_{s}=\vec{\omega}_{c}$; however, for $g \neq 2$,

$$
\vec{\omega}_{a} \equiv \vec{\omega}_{s}-\vec{\omega}_{c}=-\left(\frac{g-2}{2}\right) \frac{q \vec{B}}{m}=-a_{\mu} \frac{q \vec{B}}{m},
$$

where we have defined $\omega_{a}$ as the anomalous precession frequency. It is this quantity that must be measured to determine $(g-2)$. 
The recent storage ring experiments used electric quadrupoles to provide vertical containment - effectively creating a large Penning trap. The motional magnetic field seen by a relativistic muon in an electric field $\vec{E}$ will contribute an important term to the spin precession rate. Additionally, a nonzero muon EDM will also require modification to Eqn. 25. The full expression is then

$$
\vec{\omega}_{n e t}=-\frac{q}{m}\left[a_{\mu} \vec{B}-\left(a_{\mu}-\frac{1}{\gamma^{2}-1}\right) \frac{\vec{\beta} \times \vec{E}}{c}+\frac{\eta}{2}\left(\vec{\beta} \times \vec{B}+\frac{\vec{E}}{c}\right)\right],
$$

where $\vec{\omega}_{n e t}=\vec{\omega}_{a}+\vec{\omega}_{E D M}$.

The proposed J-PARCE34 experiment will not use electric focusing, which simplifies Eqn.26 and separates the contributions of $\omega_{a}$ and $\omega_{E D M}$. In contrast, the modern storage ring experiments operate with a relativistic gamma of 29.3, $\left(P_{\mu}=3.094 \mathrm{GeV} / c\right)$, which makes the first expression in parentheses vanish. In practice, a combined correction to $\omega_{a}$ in BNLE821 owing to the electric-field and related pitch correction was $+0.77 \pm 0.06 \mathrm{ppm} 15$.

Parity violation in the muon decay chain $\mu^{-} \rightarrow e^{-} \bar{\nu}_{e} \nu_{\mu}$ provides the necessary polarimetry that is required to access the average muon spin direction vs. time; that is, the link to $\omega_{a}$. The CM correlation between the emitted angle and energy of the decay electron with respect to the muon spin direction is illustrated in Fig. 5 . To a good approximation, the electron energy in the boosted laboratory frame is related to the energy and angular distribution in the CM as

$$
E_{e, l a b} \approx \gamma E_{e, C M}\left(1+\cos \theta_{C M}\right) .
$$

This is the key relation. The highest energy electrons are preferentially emitted when the muon spin is opposite to its momentum (alternatively, along its momentum for $e^{+}$from $\mu^{+}$decay). Every decay electron has a momentum smaller than its parent muon and consequently curls to the inside of the storage radius where a detector is positioned to intercept it and measure its energy and arrival time.

The accumulated number of electrons having an energy greater than $E_{t h}$ forms a distribution vs. time that has the structure

$$
N\left(t ; E_{t h}\right)=N_{0} e^{-t / \gamma \tau_{\mu}}\left[1+A \cos \left(\omega_{a} t+\phi\right)\right]
$$

for a $100 \%$ polarized beam. The normalization $N_{0}$ and asymmetry $A$ depend on $E_{t h}$. The ensemble-averaged spin direction at $t=0$ is represented by $\phi$, which can have a subtle energy dependence because the time of decay measured at the detector might have an energy-dependent time-of-flight component from the time of the muon decay. A representative data set from BNLE821 is shown in Fig. 21 a. The time-dilated lifetime of $\approx 64.4 \mu \mathrm{s}$ is evident, upon which is the modulation from $\omega_{a}$. The actual $N\left(t ; E_{t h}\right)$ distribution can be more

\footnotetext{
${ }^{15}$ The finite momentum spread, $\delta P_{\mu} / P_{\mu} \approx 0.15 \%$, means the term in parenthesis does not vanish completely. The vertical betatron oscillations cause $\vec{B} \cdot \vec{P}_{\mu}$ to not always equal zero.
} 
complicated compared to Eqn. 28 because of coherent betatron oscillations that give rise to further modulations of $N, A$ and $\phi{ }^{16}$ The statistical uncertainty on $\omega_{a}$ has been described in detail in Ref. [110. Different fitting methods and weighting schemes can produce reduced statistical uncertainties from the same data; however, sensitivity to leading systematic errors typically increases when one is using the more aggressive analysis procedures. The simple threshold method that is robust and tested has a relative uncertainty on $\delta \omega_{a} / \omega_{a}$ that behaves as

$$
\delta \omega_{a} / \omega_{a}=\frac{1}{\omega_{a} \gamma \tau_{\mu}} \sqrt{\frac{2}{N A^{2}\langle P\rangle^{2}}}
$$

where we include the ensemble averaged polarization $\langle P\rangle$ for completeness. To minimize $\delta \omega_{a} / \omega_{a}$, it is advantageous to: 1$)$ Use a high magnetic field $\left(\omega_{a} \propto B\right)$; 2) Run at high energy (increases $\gamma \tau_{\mu}$ ); 3) Employ a highly polarized muon source; 4) Optimize the figure-of-merit (FOM), $N A^{2}$. The latter occurs when $E_{t h} / E_{\max } \approx 0.6$.

To obtain $a_{\mu}$ from the measurement of $\omega_{a}$ requires an equally precise measurement of the magnetic field. This was accomplished in BNLE821 using a suite of pulsed NMR probes. They were used to establish the absolute field magnitude, control the time stability of the field via feedback to the magnet power supply, and to periodically map the field in situ by use of a multi-probe NMR trolley that could traverse the circumference of the storage ring orbit without breaking the vacuum. The water or petroleum jelly filled probes provided a field value in terms of the free proton precession frequency $\omega_{p}$. Minimization of the systematic uncertainty in the field integral is intimately related to the intrinsic field uniformity. Minimizing multipoles having higher order than the

1205 dipole depends on the care and precision of the shimming tasks carried out prior to physics data taking. The final azimuthally averaged magnetic field is represented by a contour map as shown in Fig.21p.

With $\omega_{a}$ and $\omega_{p}$ measured, the muon anomaly was obtained from

$$
a_{\mu}=\frac{\omega_{a} / \tilde{\omega_{p}}}{\omega_{L} / \tilde{\omega}_{p}-\omega_{a} / \tilde{\omega_{p}}}=\frac{R}{\lambda-R} .
$$

In the ratio $R \equiv \omega_{a} / \tilde{\omega}_{p}, \tilde{\omega_{p}}$ is the free proton precession frequency in the average magnetic field experienced by the muons. The muon-to-proton magnetic moment ratio $\lambda=3.183345107(84)$ is determine ${ }^{17}$ from muonium hyperfine level structure measurements [11] together with QED, see Sec.6] and Ref.[4].

\subsubsection{Fermilab muon $(g-2)$ experiment}

The precision goal of E989 is $\delta a_{\mu}=16 \times 10^{-11}$ (140 ppb), a four-fold improvement compared to BNLE821. This error includes a $100 \mathrm{ppb}$ statistical

\footnotetext{
${ }^{16}$ True for the storage ring experiments that use quadrupole focussing; absent for the JPARC E34 configuration.

${ }^{17}$ Here we quote the updated value from the 2010 CODATA recommended values of the fundamental physical constants [4].
} 
component in the measurement of $\omega_{a}$, where 21 times the data from BNL will be required. Equal systematic uncertainties of $70 \mathrm{ppb}$ are budgeted for both $\omega_{a}$ and $\omega_{p}$, corresponding to reductions by factors of 3 and 2 , respectively from what has been achieved. While BNL E821 improved on the CERN III experiment in a revolutionary manner-primarily by the invention of direct muon injection into the storage ring - the FNAL E989 experiment, in contrast, will introduce a broad suite of refinements focussed on optimizing the beam purity and rate, the muon storage efficiency, and modernizing the instrumentation used to measure both $\omega_{a}$ and $\omega_{p}$.

A limiting factor at BNL was the $120 \mathrm{~m}$ beamline between the pion pro1225 duction target and the storage ring. Because the decay length of a $3.11 \mathrm{GeV} / c$ pion is $\approx 173 \mathrm{~m}$, the beam injected into the storage ring contained both muons and a significant number of undecayed pions, the latter creating an enormous burst of neutrons when intercepting materials. Their subsequent capture in scintillator-based detectors impacted detector performance adversely. The Fermilab accelerator complex will deliver pure, high-intensity muon bunches to the storage ring at a fill-rate frequency increase of $\sim 3$ compared to BNL. Proton batches at $8 \mathrm{GeV}$ from the Booster are divided into short bunches in the Recycler Ring. Each bunch is then extracted and strikes a target station tuned to collect $3.1 \mathrm{GeV} / c \pi^{+}$and transport them along a $270 \mathrm{~m}$ FODO ${ }^{18}$ lattice. The

1235 highest energy decay muons in the $\pi^{+} \rightarrow \mu^{+} \nu_{\mu}$ decay chain are captured and transported along the same beamline with a longitudinal polarization of $\sim 97 \%$. These muons (together with pions and protons) are injected into the repurposed $\bar{p}$ Delivery Ring (DR). There, they make several revolutions to reduce the pion contamination by decay and to separate protons by their velocity difference.

${ }_{1240}$ A kicker in the DR extracts the pure muon bunch into a short beamline that terminates at the storage ring entrance.

The centerpiece of the experiment is the $1.45 \mathrm{~T}$ superconducting storage ring [112 that was re-located to Fermilab from Brookhaven in 2013. It has recently been reassembled in a custom temperature-controlled building having

1245 a firm foundation for the magnet support; both are critical for the magnetic field stability. Three storage ring subsystems - the superconducting inflector, four electric quadrupoles, and a fast kicker - determine the fraction of incoming muons $\epsilon_{\text {store }}$ that become stored, and their subsequent beam properties such as betatron oscillations. Improvements and replacements in these devices are aiming a factor of 2 or more increase in $\epsilon_{\text {store }}$ compared to BNL.

The storage ring magnetic field will be shimmed following procedures developed for E821, with small improvements owing to the need for a more highly uniform final field. Retooling of the pulsed NMR probes, modern 3D OPERA model guidance, upgrades to the in-vacuum shimming trolley, and NMR probe 1255 readout using waveform digitizers, represents just some of the work. The absolute NMR probe is the same one used in the muonium hyperfine experiment

\footnotetext{
${ }^{18}$ alternating pairs of focusing and defocusing quadrupoles magnets tuned to transport the pions and the forward-decay muons
} 

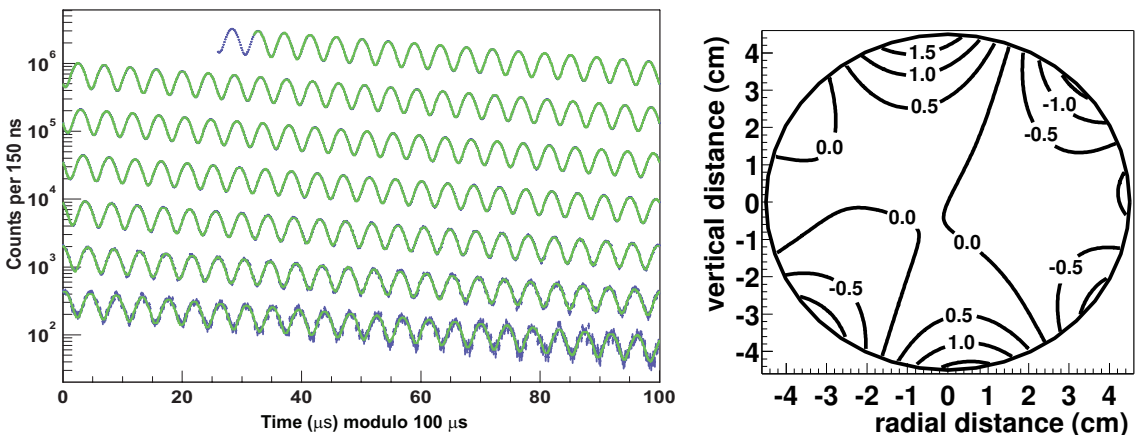

Figure 21: Left: E821 anomalous precession data, including fit. The data is wrapped around every $100 \mu \mathrm{s}$. Right: The final, azimuthally averaged magnetic field contours; the scale is in ppm with respect to the average. Figures courtesy E821 collaboration.

that established the muon-to-proton magnetic moment ratio $\lambda$ in Eqn. 30 and it will be cross calibrated with the new J-PARC experiment (see Sec. 6) New absolute probes are also being developed as cross checks.

The entire suite of detectors, electronics, calibration and data acquisition systems will be new and modern. These include highly segmented lead-fluoride Cherenkov calorimeters, where each crystal is read out by silicon photomultipliers 113. The signals are recorded using custom $800 \mathrm{MHz}, 12$-bit-depth waveform digitizers. A distributed laser-based calibration system is designed to maintain gain stability at the $0.04 \%$ level. Several stations of in-vacuum straw tracking detectors will provide beam dynamics measurements that are required to control various systematic uncertainties. Finally, a high-speed, GPU-based DAQ is prepared to process Gbytes/s of data in a deadtime free operation.

The systematic uncertainty estimates are based on the considerable experience of E821 and the targeted experimental upgrades that have been made to address each one of them. At the time of this review, the storage ring is being commissioned, with a 9-month shimming period planned for 2015. The beamlines are being built, and the detector and electronics systems have undergone various test-beam runs at Fermilab and SLAC. Physics data taking is expected to begin in 2017.

\subsubsection{J-PARC muon ( $g$-2) experiment}

While the initial $\delta a_{\mu}$ statistical precision goal of the proposed J-PARC experiment was also at the $100 \mathrm{ppb}$ level, current estimates based on the muon source intensity and polarization now aim at $\sim 400 \mathrm{ppb}$, similar to the BNL experiment 114. The collaboration is developing a creative new method that will feature different systematic uncertainties compared to the storage ring experiment; thus, the systematic error budget is difficult to anticipate at this time.

The most striking difference will be the use of a 10-times lower momentum, but ultra-cold, muon beam. This choice is motivated by the desire to elim- 
inate the electric field - and thus the spin-precession terms that are affected by it - used for vertical focusing in storage rings. To accomplish this goal, a muon beam must be made with a negligible transverse momentum component, $\Delta P_{T} / P_{\mu} \approx 10^{-5}$. This is accomplished by accelerating from rest a source of ultra-cold muons created by the re-ionization of muonium atoms in vacuum. How the overall experiment is then designed, both upstream - creating the muon source - and downstream - where the positrons are measured - is quite unique.

A $3 \mathrm{GeV}$ proton beam strikes a graphite target, producing pions that can decay at rest near to the surface of the target. The $28.4 \mathrm{MeV} / c, 100 \%$ longitua polarized $\mu^{+}$surface muon beam is directed to a thin target optimized to stop muons and form muonium atoms, $M \equiv \mu^{+} e^{-}$. The target is designed to permit muonium to diffuse into the vacuum on the downstream surface. Aggressive efforts over the past few years have realized promising results in raising the net yield of muonium that emerges into the vacuum. A recent study at TRIUMF used a silica aerogel target where micro-channels in the target were created using a laser ablation technique 114. This target resulted in an 8-fold improvement compared to the previous measured yield using the same material. When the J-PARC beam rates are combined with the TRIUMF muonium yield measurements, an expected production of $0.2 \times 10^{6} / \mathrm{s}$ is found. While a factor 1305 of 5 lower than originally planned, it still represents a major step forward and a viable rate for a $(g-2)$ experiment. Studies will continue aimed at further rate improvements.

The muonium atom 115 and its hyperfine structure measurements are described in Sec. 6, we also direct the reader to Fig. 25, which illustrates the external field dependent quantities discussed herein. Muonium formed in the weak magnetic field limit can be described in terms of its total angular momentum and associated magnetic quantum numbers $\left(F, M_{F}\right)_{i}$, where the triplet and singlet combinations are given as: $(1,1)_{1},(1,0)_{2},(1,-1)_{3}$ and $(0,0)_{4}$, and the subscript $i$ is our shorthand label for the four states. If muonium is formed in zero magnetic field, the relative population of the four states $i=1$ to 4 is: $\frac{1}{2}, \frac{1}{4}, 0$ and $\frac{1}{4}$, respectively. Allowing for a weak field, and choosing the axis of quantization along the incoming muon polarization direction, $\hat{z}$, the net muon polarization as a function of time is given by [116]

$$
P_{z}=\frac{1}{2}\left(\frac{1+2 x_{B}^{2}+\cos 2 \pi \nu_{24} t}{1+x_{B}^{2}}\right) \rightarrow \frac{1}{2}\left(1+\cos 2 \pi \nu_{24} t\right) \quad \text { as } \quad x_{B} \rightarrow 0 .
$$

Here, the field strength is traditionally expressed by $x_{B}$, which is a ratio of the sum of the electron and muon Zeeman interactions to the muonium ground-state 1310 hyperfine interval, all expressed in terms of frequencies. To obtain a sample of at-rest, but polarized muons, the muonium atoms must be re-ionized, which is accomplished in the vacuum by two simultaneous laser bursts having wavelengths $\lambda_{1}=122 \mathrm{~nm}$ and $\lambda_{2}=355 \mathrm{~nm}$. The first excites the $1 \mathrm{~S}$ to $2 \mathrm{P}$ transition and the second ionizes the atom, leaving the free $\mu^{+}$essentially at rest in the vacuum. Muons ionized from state 1 , the $(1,1)$ triplet, retain their initial polarization. In contrast, those populating states 2 and 4 can make transitions at the 
rate $\nu_{24} \approx 4.5 \mathrm{GHz}$, which is so high that the emerging muon spin will be, for all practical purposes, unpolarized. Consequently, the maximum net polarization from the muonium-at-rest source is $P_{z}=50 \%$.

${ }_{1320}$ The ultra-cold, "at rest," muons will be rapidly accelerated by a linac to a longitudinal momentum of $\vec{P}_{\mu}=300 \mathrm{MeV} / c$. A novel feature of E34 is that the polarization direction can be flipped at production, prior to acceleration, by use of a low magnetic field. This feature might become important for anticipated systematic uncertainties.

${ }_{1325}$ The muon beam will be directed through the top of a highly uniform $3 \mathrm{~T}$ MRI-type magnet. A spiral injection path allows the muons to enter the field region at a steep angle, which softens such that the beam eventually orbits a plane perpendicular to the magnetic field, see Fig. 22, Custom fringe-fields and a vertical kicker are key elements in the design. Very weak magnetic focusing is required, but it causes a negligible perturbation to the $\omega_{a}$ frequency.

Apart from the kinematic differences and uniqueness of the source, once the muons begin to circulate in the field, the experiment is much like the higherenergy storage ring designs. The muon spin precesses proportionally to $(g-2)$ and the anomalous precession frequency is encoded in the modulation of rate

1335 vs. time of the higher-energy positron decays.

The muon momentum and field strength values imply that the orbit radius is $33 \mathrm{~cm}$ and the cyclotron period is $7.4 \mathrm{~ns}$. Decay positrons curl to the inside of the central orbit where vanes of silicon strip detectors are positioned. The radius of the reconstructed positron tracks provides the momentum (energy)

1340 determination with good acceptance, and the location of the detectors is tuned for good performance for the higher-energy range that optimizes the FOM. The detector system must have a stable acceptance over the measuring period and withstand a total initial hit rate approaching $10^{9}$ hits/s [117. Sorting of hits into tracks presents a unique challenge here. However, if solvable, the 1345 systematics of complete track reconstruction could be lower than those inherent using calorimeter techniques, and in any case, they will be different.

\subsubsection{Comparison of methods}

A comparison of many of the parameters between the two new $(g-2)$ experiments is shown in Table 4. Equation 29 can be used to evaluate the number 1350 of required events necessary to meet the desired statistical precision. We used a polarization of $50 \%$ for the J-PARC muonium beam and $97 \%$ for the decayin-flight beam at Fermilab.

\subsection{Electric Dipole Moment}

A permanent electric dipole moment (EDM) of a particle or fundamental 1355 system violates the discrete symmetries of parity $(\mathrm{P})$ and time-reversal $(\mathrm{T})$. Because quantum field theories are CPT invariant, a T-violation observation leads to $\mathrm{CP}$ violation (CPV). Finding a new source of CPV is a major quest in atomic, nuclear and particle physics because of its implications in any resolution of the baryon-antibaryon asymmetry problem: $\left(n_{B}-n_{\bar{B}}\right) / n_{\gamma}=6 \times 10^{-10}$ excess 


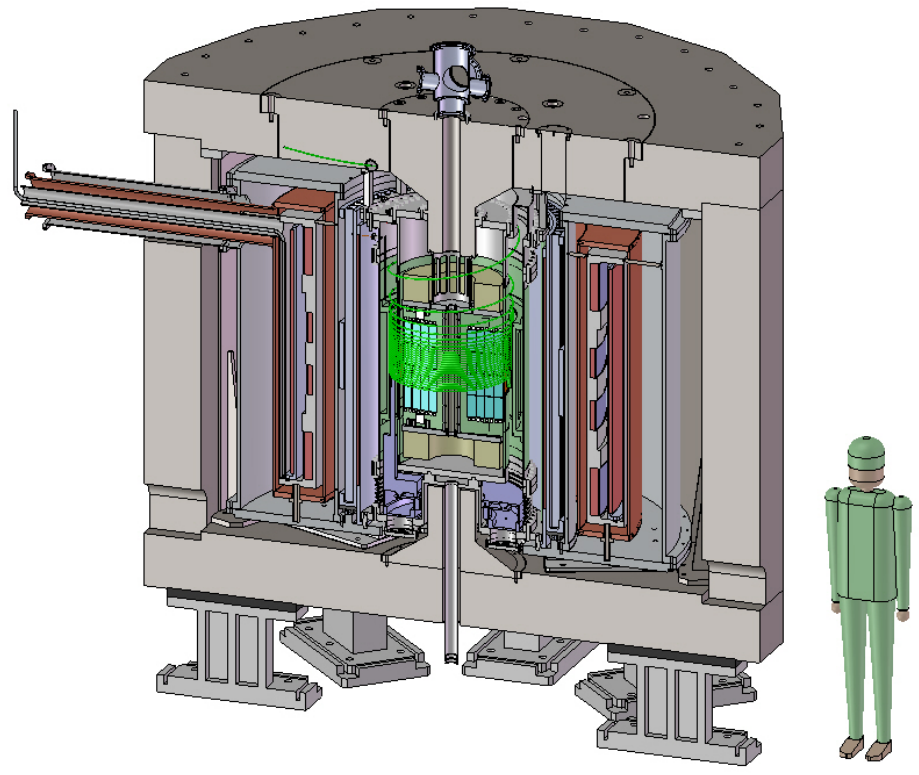

Figure 22: The proposed setup for the J-PARC $(g-2)$ Experiment. Muons enter at the top left (green trajectory) and spiral into the highly uniform magnetic field region. Their decay positrons curl inward to an array of silicon tracking detectors. Figure courtesy T. Mibe.

Table 4: Comparison of various parameters for the Fermilab and J-PARC $(g-2)$ Experiments

\begin{tabular}{lcc}
\hline Parameter & Fermilab E989 & J-PARC E24 \\
\hline Statistical goal & $100 \mathrm{ppb}$ & $400 \mathrm{ppb}$ \\
Magnetic field & $1.45 \mathrm{~T}$ & $3.0 \mathrm{~T}$ \\
Radius & $711 \mathrm{~cm}$ & $33.3 \mathrm{~cm}$ \\
Cyclotron period & $149.1 \mathrm{~ns}$ & $7.4 \mathrm{~ns}$ \\
Precession frequency, $\omega_{a}$ & $1.43 \mathrm{MHz}$ & $2.96 \mathrm{MHz}$ \\
Lifetime, $\gamma \tau_{\mu}$ & $64.4 \mu \mathrm{s}$ & $6.6 \mu \mathrm{s}$ \\
Typical asymmetry, $A$ & 0.4 & 0.4 \\
Beam polarization & 0.97 & 0.50 \\
Events in final fit & $1.5 \times 10^{11}$ & $8.1 \times 10^{11}$ \\
\hline
\end{tabular}


baryons per photon in the present universe, where $n_{B}+n_{\bar{B}}=0$ is assumed at the Big Bang. The three Sakharov conditions required to arrive at an excess of baryons are: 1) at least one $B$-number violating process; 2) a source of Cand CP-violation; and 3) interactions that occur outside of thermal equilibrium. The challenging task of knitting together these ingredients into a quantitatively complete explanation is not yet complete and the reader is referred to discussions of this fascinating topic; see [118, 119. One important fact, is that the $\mathrm{CP}$ violation that occurs in the lone phase in the CKM mixing matrix is insufficient by many orders of magnitude to explain a mechanism for baryogenesis. Consequently, a new source of CPV is required. Vigorous experimental efforts

1370 are underway at quark-flavor factories, in neutrino oscillation experiments, and in EDM searches using atoms, molecules and neutrons.

We begin with a caveat. In a hadronic system, an EDM can be accommodated within the SM owing to the CP-violating $\Theta_{\mathrm{QCD}}$ term. The nonobservation of a neutron EDM with $d_{n}<10^{-26} e \cdot \mathrm{cm}$, implies $\Theta_{\mathrm{QCD}}<10^{-10}$.

1375 The SM-allowed term appears to be very finely tuned - the strong CP problemif it is finite at all. The situation can be completely resolved if the physical axion exists; the Peccei-Quinn mechanism allows $\Theta_{\mathrm{QCD}}=0$ at the price of a new particle [43. An axion of the right mass is also highly motivated to fulfil the role of the missing dark matter particle and current searches by the ADMX collaboration will soon largely explore that parameter space completely. 43.

New sources of CPV are not unexpected in popular extensions of the standard model, such as supersymmetry, and two-Higgs doublet models (see Refs. and discussion in [119). Current experimental limits are listed in Table 5. The non-observations are beginning to have severe consequences on all of the models. For example, they already severely constrain many supersymmetric $\mathrm{CP}$-violating phases. In the simple lepton sector, the electron imbedded in a polar molecule is probed to very impressive limits. However, the fundamental source of any observed CPV in this kind of a system can have multiple interpretations as to its origin [120. Is it singularly from the electron? The muon, 1390 on the other hand, is the only fundamental particle that can be directly tested and therefore easiest to interpret. It is also the only 2nd-generation particle being probed, which can have important implications in certain BSM models. In general, the sensitivity to new physics is expected to scale linearly owing to the mass term in the denominator of the dipole moment definition. Current $d_{e}$ limits are then nearly 7 orders of magnitude more sensitive to new physics than the muon.

However, there are BSM scenarios in which non-linear scaling occurs. Babu et al. 121. presented an argument for $\left(m_{\mu} / m_{e}\right)^{3}$ scaling, a $\sim 10^{7}$ fold enhancement, meaning a next-generation muon EDM search would be competitive to

1400 the electron. This analysis deserves an update based on new information from lepton-flavor-changing tau decay limits, the improved electron EDM, and direct LHC bounds, but it remains an intriguing consideration.

More recently, Hiller et al. 122 proposed a supersymmetric model with $\mathrm{CP}$ violation from lepton flavor violation that can achieve rather large values 1405 for $d_{\mu}$-as large as $10^{-22} e \cdot \mathrm{cm}$ - in the extremes of the model parameter space. 
Table 5: Selected EDM limits for the electron, $\mathrm{Hg}$ atom, neutron and muon.

\begin{tabular}{cclc}
\hline Type & System & EDM Limit $(\mathrm{e}-\mathrm{cm})$ & Ref. \\
\hline Paramagnetic & YbF & $d_{e}=(-2.4 \pm 5.9) \times 10^{-28}$ & {$[124$} \\
Paramagnetic & ThO & $d_{e}=(-2.1 \pm 4.5) \times 10^{-29}$ & {$[125]$} \\
Diamagnetic & ${ }^{199} \mathrm{Hg}$ & $d_{A}=(0.5 \pm 1.5) \times 10^{-29}$ & {$[126$} \\
Nucleon & Neutron & $d_{n}=(0.2 \pm 1.7) \times 10^{-26}$ & {$[127$} \\
Lepton & Muon & $d_{\mu}=(-0.1 \pm 0.9) \times 10^{-19}$ & {$[7]$} \\
\hline
\end{tabular}

The practical range is constrained by the limits on the flavor-mixing decay $B R(\tau \rightarrow \mu \gamma)<10^{-8}$, which will be improved with Belle II running in the future; lower BR's there imply smaller $d_{\mu}$.

The searches for SUSY at the colliders primarily focus on $R$-parity conserving models. Considering $R$-parity-violating (RPV) models opens up the parameter space considerably [123. In such an analysis, it is observed that the muon is unique from the other systems being probed and limits as high as $d_{\mu} \sim 10^{-24} e \cdot \mathrm{cm}$ can be imagined. While these very different examples do predict relatively "large" values for $d_{\mu}$, the relevant range sets a challenging experimental goal for muon enthusiasts as we discuss below.

\subsubsection{Experimental Considerations}

A typical atomic, molecular, or neutron EDM experiment involves a measurement of the difference in the spin precession frequency of a system subject to parallel and, alternatively, antiparallel magnetic and electric fields. In practice, it is vital to work with the strongest possible electric fields, which are often found in the interior of atoms or molecules, where they greatly exceed laboratory capabilities. For a relativistic muon circulating in a plane orthogonal to a pure dipole magnetic field, the situation is quite different. Here, the muon will feel a transverse induced motional electric field $\vec{E}_{m} \propto \vec{\beta} \times \vec{B}$. For the $(g-2)$ storage rings, where $\gamma=29.3$, the electric field strength is nearly $13 \mathrm{GV} / \mathrm{m}$ !

To understand the measurement concept, we rewrite Eqn. 26 in the absence of an external electric field. While J-PARC will not use one at all, the focussing electric field at Fermilab has $(E / c \ll B)$ and the $\gamma$ is selected to eliminate the affect on the precession of the spin. Thus, we have simply

$$
\vec{\omega}_{n e t}=\vec{\omega}_{a}+\vec{\omega}_{E D M}=-\frac{q}{m}\left[a_{\mu} \vec{B}+\frac{\eta}{2}(\vec{\beta} \times \vec{B})\right] .
$$

The precession orientations for the magnetic and electric moments are orthogonal, as illustrated in Fig. 23. For a non-zero EDM, the precession plane would be tilted inward toward the center of the cyclotron orbit by the very small angle

$$
\delta=\tan ^{-1}\left(\frac{\omega_{E D M}}{\omega_{a}}\right)=\tan ^{-1}\left(\frac{\eta \beta}{2 a_{\mu}}\right)
$$

and the observed precessional frequency would be $\omega_{t o t}=\sqrt{\omega_{E D M}^{2}+\omega_{a}^{2}}$. The key for the experimentalist is that the tilt of the plane is the signal. 


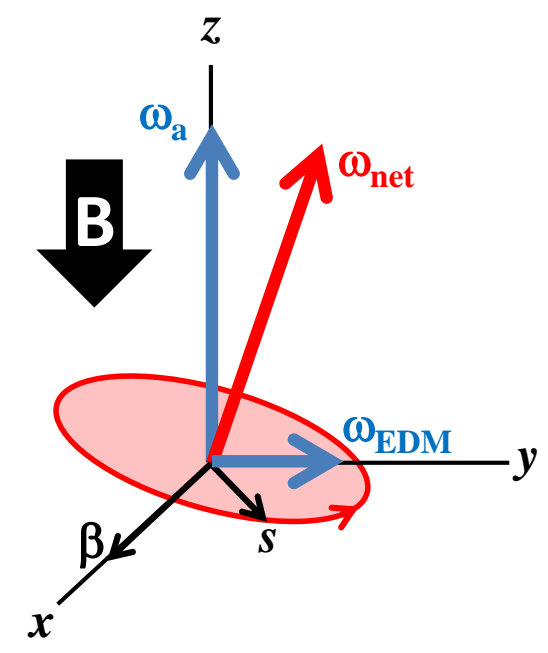

Figure 23: The net precession frequency is a vector sum of $\omega_{a}$ caused by the anomalous magnet moment and $\omega_{E D M}$ caused by a possible permanent electric dipole moment. For a $\mu^{+}$in the $\hat{x}$ direction, its spin would rotate counterclockwise in the $x-y$ plane in the absence of an EDM. The $\beta \times \vec{B}$ term in Eqn. 26 points along the $\hat{y}$ axis, orthogonal to $\omega_{a}$. This tips the precession plane as shown. Note, $\left|\omega_{E D M}\right| \ll\left|\omega_{a}\right|$ in practice. It is exaggerated in the figure for clarity.

Because the muon spin reverses every half period, the direction in which it is tipped also reverses. The consequence is that the observable will be an up/down modulation of the decay particles that is out-of-phase with the spin orientation by a factor of $\pi / 2$. In practice, the most precise method of determining the tilt is a measurement of the average slope of the decays - upward vs downwardvs. time. The trajectories can be precisely determined using a set of tracking chambers.

The BNL experiment found $d_{\mu}=(-0.1 \pm 0.9) \times 10^{-19} e \cdot \mathrm{cm}$ using a limited subset of the data and measured at just 1 of the 24 detector stations [7]. Both new $(g-2)$ experiments will be sensitive to an EDM at a level close to $10^{-21} e \cdot \mathrm{cm}$, a major improvement. In each case, they will rely on the up/down slope asymmetry using trackers. Here, the J-PARC experiment, which is an

1440 all-tracker detector, should have a greater overall acceptance compared to the FNAL experiment, which will feature at first only 3 tracker stations in the 24 discrete detector positions.

In the parasitic method described above, the rapid precession of the magnetic moment reverses the upward and downward tipping of the spin owing to

1445 a possible EDM on every cycle. This leaves at most a very faint modulating signal at the frequency $\omega_{n e t} \approx \omega_{a}$. In contrast, dedicated storage-ring measurements of EDMs have been proposed by Farley et al. [128] using the "frozen spin" technique. In this method, the $\omega_{a}$ precession is set to zero in crossed vertical 
magnetic and radial electric fields for a proper selection of field magnitudes and muon momentum. If $\eta \neq 0$, the particle spin will gradually tip out of plane (upward or downward) precessing about the radial electric field, greatly enhancing the signal. A compact muon EDM experiment designed on this principle has been suggested by Adelmann et al [129.

The net spin precession in Eqn. 26, when $\eta=0$, is frozen when

$$
-\frac{q}{m}\left[a_{\mu} \vec{B}-\left(a_{\mu}-\frac{1}{\gamma^{2}-1}\right) \frac{\vec{\beta} \times \vec{E}_{r}}{c}\right]=0,
$$

which occurs when the external radial electric field $E_{r}$ has the magnitude

$$
E_{r}=\frac{a_{\mu} B c \beta}{\left.1-\left(1+a_{\mu}\right) \beta^{2}\right)} \approx a_{\mu} B c \beta \gamma^{2} .
$$

Using the realistic set of parameters- $E_{r}=640 \mathrm{kV} / \mathrm{m}, p_{\mu}=125 \mathrm{MeV} / c, B=$ $1 \mathrm{~T}$ - the authors of 129 predict a sensitivity of $\delta d_{\mu} \approx 7 \times 10^{-23} \mathrm{e} \cdot \mathrm{cm}$ with 1 year of running at PSI on an available muon beamline. The challenges of injection into this unique and compact device, $\left(R_{\text {cyclotron }}=42 \mathrm{~cm}\right)$, and the potential systematics that can lead to spin tipping from effects unrelated to an EDM are discussed in their paper, and also in Ref. [128. While remaining

1460 many orders of magnitude behind the linearly-scaled $d_{e}$ established limits, it is still an idea worth keeping alive. If we are to fully understand any non-zero EDM, we will need many probes to decouple the various interpretations from fundamental CPV sources. This small-scale experiment would serve well as a general demonstration of the storage-ring based EDM proposals that have been

1465 extended to focus on the deuteron and the proton, with promises in those cases of very impressive limits.

\section{Muonium hyperfine structure}

\subsection{Experimental approaches to muonium spectroscopy}

Muonium $\left(\mu^{+} e^{-}\right)$is the electromagnetic bound state of a positive muon and 1470 a negative electron. It is a purely-leptonic, hydrogen-like atom that unlike either ordinary hydrogen or muonic hydrogen is completely free from the complications associated with the proton's finite size and its electromagnetic sub-structure.

Muons and electrons are spin- $1 / 2$ particles and consequently the muonium $1 S$ ground state has a hyperfine structure that comprises a spin $F=1$ triplet 1475 state with three magnetic substates $M_{F}=-1,0,+1$ and a spin $F=0$ singlet state with one magnetic substate $M_{F}=0$. The interaction between the magnetic dipole moments of the muon and the electron causes an energy splitting between the $F=0,1$ hyperfine states of about $18 \mu \mathrm{eV}(4.5 \mathrm{GHz})$. As shown in Fig. 24 in the presence of a static magnetic field - the Zeeman effect causes a further splitting of the hyperfine states, with the Breit-Rabi equation describing the energy levels versus field strength (for example see Refs. [130, 131]). In 


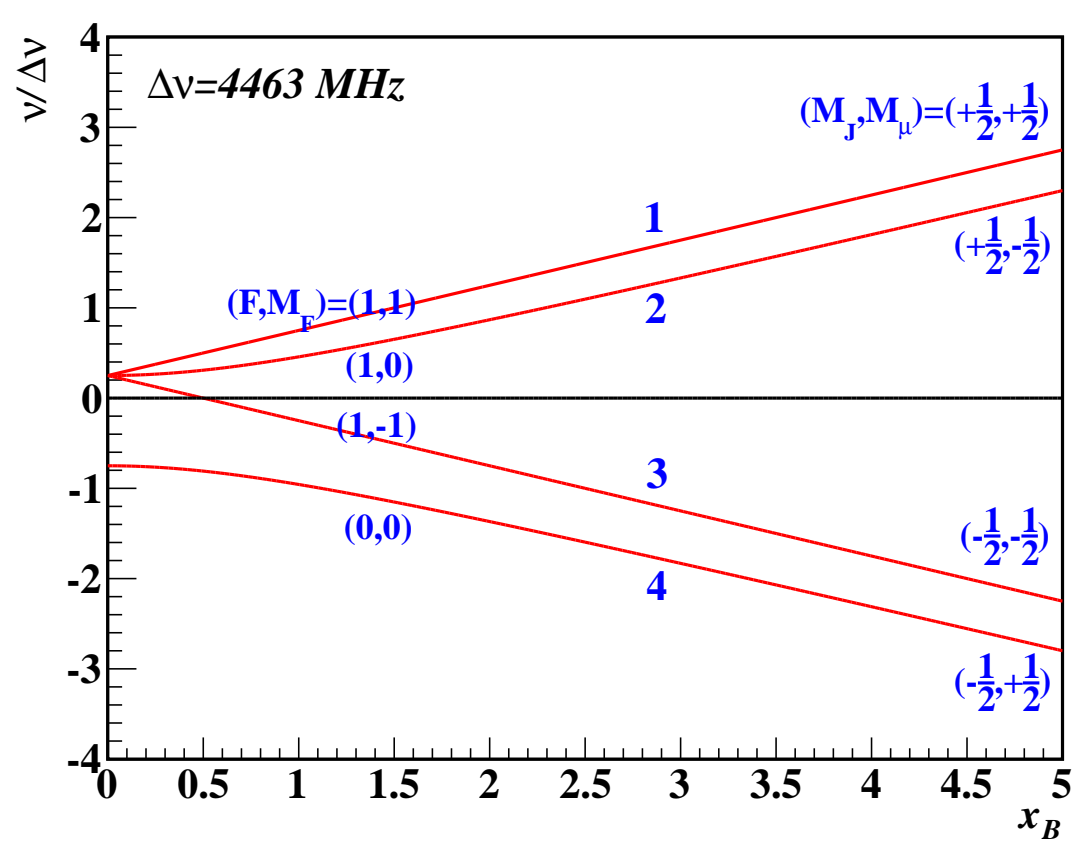

Figure 24: Breit-Rabi diagram of the energy levels of the muonium $1 S$ hyperfine states versus the field strength parameter $x_{B}=\left(g_{J} \mu_{B}^{e}+g_{\mu}^{\prime} \mu_{B}^{\mu}\right) H / h \Delta \nu$ where $g_{J}, g_{\mu}^{\prime}$ are the electron and muon gyromagnetic ratios in muonium, $\mu_{B}^{e}, \mu_{B}^{\mu}$ are the electron and muon Bohr magnetons, $\Delta \nu$ is the hyperfine interval, and $\mathrm{H}$ is the field strength. The labels $1 \ldots 4$ denote the two Zeeman transitions $\left(\nu_{12}\right.$ and $\left.\nu_{34}\right)$ that are measured in the LAMPF and MuSEUM hyperfine structure experiments.

the weak-field limit the magnetic interaction between the muon-electron magnetic moments dominates and the aforementioned $\left(F, M_{F}\right)$ are good quantum numbers. In the strong-field limit the magnetic interaction with the applied magnetic field dominates and the electron and muon spin projections $\left(M_{J}, M_{\mu}\right)$ are good quantum numbers.

Muonium was identified in 1960 by the groups of Hughes at Columbia and Telegdi at Chicago [132, 24]. The experiments detected the characteristic signal of muonium precession in a weak field by recording the high-energy positrons 1490 emitted from polarized muon stops in argon gas ${ }^{19}$ In these circumstances the muonium atoms were directly formed in their $1 \mathrm{~S}$ ground state by electron capture from argon atoms. Since this work, the formation of muonium has been

\footnotetext{
${ }^{19}$ In such "weak field" experiments, using polarized muons and unpolarized electrons, the different $\left(F, M_{F}\right)$-states are populated in proportions $(1,+1)=1 / 2,(1,0)=1 / 4,(1,-1)=0$, and $(0,0)=1 / 4$. Such experiments thereby observe the precession of the muonium atoms formed in the $\left(F, M_{F}\right)=(1,+1)$ hyperfine state.
} 
observed in many other materials, as well as produced in vacuum through thermal emission from hot metal foils and fine silica powders.

After the discovery of muonium much attention was focused on the hyperfine structure of its $1 \mathrm{~S}$ ground state. The first estimates of the hyperfine splitting were derived from measurements of the muonium polarization versus the applied field ${ }^{20}$ Soon afterwards precision experiments involving microwave resonance techniques were used to induce transitions between different hyperfine states and thereby determine the hyperfine structure. The hyperfine transitions were detected through the associated muon spin-flip and the corresponding change in the decay positron angular distribution.

In low-field resonance experiments, the transition frequency directly determines the hyperfine interval, $\Delta \nu$. In high-field resonance experiments, the determination of the hyperfine interval from the measurement of a single transition frequency requires the use of the Breit-Rabi equation and knowledge of the muon magnetic moments. This issue motivated the development by DeVoe et al. [133] of a so-called "double-resonance" technique involving the high-field measurement of two hyperfine transition frequencies using a single microwave 1510 cavity. The technique allows the concurrent determination of both the hyperfine interval and the muon magnetic moment.

More recently, muonium hyperfine spectroscopy experiments have employed setups that alternate between two microwave fields to obtain the two hyperfine frequencies. They yield the best determinations of both the muon-to-proton 1515 magnetic moment ratio $\mu_{\mu} / \mu_{p}$ and the muon-to-electron mass ratio $m_{\mu} / m_{e}-$ two fundamental constants of great importance to precision spectroscopy of muonic atoms. The ratio $\mu_{\mu} / \mu_{p}$ is also crucial to the extraction of the muon anomalous magnetic moment, $a_{\mu}$, in the muon $(g-2)$ experiments (see Sec. 5).

Note that the hyperfine interval is both measurable and also calculable with extraordinary precision (see Ref. 4] for details of theoretical calculations). With input of other fundamental constants - most importantly the fine structure constant and the Rydberg constant - the comparison between measured and calculated values of the hyperfine interval is considered a definitive test of QED theory in bound-state systems ${ }^{21}$

\subsection{LAMPF hyperfine structure experiment}

The most recent measurement of the muonium 1S ground state hyperfine structure was conducted by Liu et al. [111 at LAMPF. The experiment measured the frequencies of the two high-field, muon spin-flip transitions $\left(M_{J}, M_{\mu}\right)$

\footnotetext{
${ }^{20}$ The muonium polarization is a function of the comparative sizes of the interaction energy of the applied field and the hyperfine splitting of the $1 \mathrm{~S}$ ground state.

${ }^{21}$ Although not discussed in detail here, the $1 S-2 S$ interval in muonium is also measured. The experiment 134] -utilizing Doppler-free, two-photon, pulse laser spectroscopy-yielded a $4 \mathrm{ppb}$ determination of $\Delta \nu_{1 S 2 S}$ in good agreement with theory. Using the combination of the results from the hyperfine experiment and the $1 S-2 S$ experiment, the authors obtained a verification of charge equality between muons and electrons of $1+q_{\mu^{+}} / q_{e^{-}}=(-1.1 \pm 2.1) \times$ $10^{-9}$.
} 


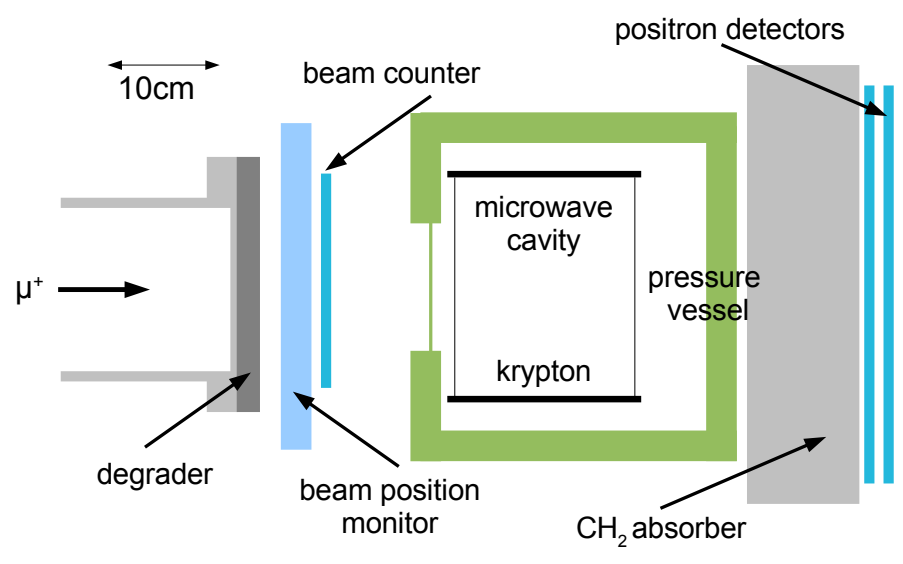

Figure 25: Schematic diagram of the LAMPF HFS experiment 111 indicating the muon beam, beam counter and profile monitor, krypton target, microwave cavity, pressure vessel and downstream high-energy positron detector system. Figure courtesy Liu et al.

$=(+1 / 2,+1 / 2) \leftrightarrow(+1 / 2,-1 / 2)$ and $(-1 / 2,-1 / 2) \leftrightarrow(-1 / 2,+1 / 2)$, denoted respectively as $\nu_{12}$ and $\nu_{34}$ in Fig. 24. The experiment employed the doubleresonance technique with a novel line-narrowing approach using a custom timestructured muon beam. The setup - including the muon beam, gas target, microwave cavity and positron detector - is depicted in Fig. 25.

The experiment used a high rate, 100\%-longitudinally polarized, surface muon beam derived from the $120 \mathrm{~Hz}$ repetition-rate, $650 \mu$ s pulse-period, LAMPF primary proton beam. An electrostatic kicker was used to produce a cycle of $4 \mu \mathrm{s}$ beam-on accumulation periods followed by $10 \mu \mathrm{s}$ beam-off measurement periods. The beam extinction between accumulation periods was roughly $99 \%$.

The incident muons entered a large-bore, high-uniformity, $1.7 \mathrm{~T}$ magnet containing a copper microwave cavity filled with pure krypton gas. Muon stops formed polarized, ground state muonium-the $\left(M_{J}, M_{\mu}\right)=(1 / 2,-1 / 2)$ and $(-1 / 2,-1 / 2)$ states - by electron capture from krypton atoms. A doublelayered scintillator telescope recorded the high-energy decay positrons that were emitted downstream of the stopping target. A combination of plastic scintillators and wire chambers were used for beam monitoring.

The microwave cavity was designed to resonate at both the $\nu_{12}$ transition frequency of $1897.5 \mathrm{MHz}$ and the $\nu_{34}$ frequency of $2565.8 \mathrm{MHz}$. NMR magnetometry using multiple fixed and movable probes was used to monitor the magnetic field.

${ }_{1550}$ When precisely tuned to $\nu_{12}$ or $\nu_{34}$ the microwave field induces muon spin-flip transitions and thereby changes the angular distribution of decay positrons. In earlier measurements all positrons are detected, both those from "fast decays" 
where the muon interacts only briefly with the microwave field and those from "slow decays" where the muon interacts at length with the microwave field. Liu et al. utilized the $4 \mu \mathrm{s}$ beam-on, $10 \mu \mathrm{s}$ beam-off, time structure to only observe the decay positrons from long-lived muonium with extended microwave interactions. This procedure narrowed the resonance lineshape by roughly a factor of three.

The positron data was collected in super-cycles of ten beam pulses with the microwave cavity alternately switched between on and off and the microwave frequency alternately tuned to $\nu_{12}$ and $\nu_{34}$. The positron signal for deriving the transition frequencies was defined as

$$
S(\nu, H)=\left(N_{o n} / N_{o f f}-1\right)
$$

where $N_{\text {on }}\left(N_{\text {off }}\right)$ represents the positron counting rates with the microwave 1560 field on (off) and $H$ and $\nu$ are the static field strength and the microwave frequency, respectively. Near the Zeeman resonances the spin-flip transitions caused a large increase in $S(\nu, H)$.

Resonance curves were collected by (i) sweeping the static magnetic field at a fixed microwave frequency and (ii) sweeping the microwave frequency at a fixed static magnetic field. The resonance curves $S(\nu, H)$ were then fit to lineshapes to extract the transition frequencies. The fitted lineshapes incorporated the muon stopping distribution, static field distribution, microwave power distribution, and positron detection efficiency. Data were collected at two gas pressures and extrapolated to zero pressure; the procedure accounted for a slight shift 1570 of the resonance frequency due to muonium-atom collisions and the resulting wavefunction distortions.

The measured resonance curves determined the two Zeeman frequencies to precisions of $17-18 \mathrm{ppb}$ (the statistical errors on the $S(\nu, H)$ resonance curves were the dominant experimental uncertainties). Using the Breit-Rabi Eqn. and the values for $\nu_{12}$ and $\nu_{34}$ a hyperfine interval of

$$
\Delta \nu=4463302765(53) \mathrm{Hz} \quad(12 \mathrm{ppb})
$$

and a magnetic moment ratio of

$$
\mu_{\mu} / \mu_{p}=3.18334513(39) \quad(120 \mathrm{ppb})
$$

were obtained. The results were a three-fold improvement over the earlier experimental work.

As mentioned earlier, an improved value for the mass ratio $m_{\mu} / m_{e}$ can be obtained from the measured value for the ratio $\mu_{\mu} / \mu_{p}$ with the input of the muon anomalous magnetic moment $a_{\mu}$ and the proton-to-electron magnetic moment ratio $\mu_{p} / \mu_{B}$. The precise measurement of $\mu_{\mu} / \mu_{p}$ thus yielded a precise determination of the mass ratio

$$
m_{\mu} / m_{e}=206.768277(24) \quad(120 \mathrm{ppb})
$$

The hyperfine structure of $1 S$ muonium thus renders the best determinations of 1575 both the magnetic moment ratio $\mu_{\mu} / \mu_{p}$ and the lepton mass ratio $m_{\mu} / m_{e}$. 
The current status of theoretical calculations of $\Delta \nu$ is given in Ref. [4. Although the largest theoretical uncertainties arise from recoil correction terms, the overall uncertainty in the $\Delta \nu$ prediction is the aforementioned experimental knowledge of the mass ratio $m_{\mu} / m_{e}{ }^{22}$ The corresponding calculated and measured values of the hyperfine interval are in good agreement within their respective uncertainties of $272 \mathrm{~Hz}(61 \mathrm{ppb})$ and $53 \mathrm{~Hz}(12 \mathrm{ppb})$. This agreement is considered an important verification of bound-state QED calculations; the precision being much greater than analogous comparisons in ordinary hydrogen and muonic hydrogen.

Alternatively - by equating the theoretical expression and measured value for the hyperfine interval $\Delta \nu$ and regarding $m_{\mu} / m_{e}$ as a free parameter-an indirect determination of the muon-to-electron mass ratio and the muon-toproton magnetic moment ratio

$$
\begin{gathered}
m_{\mu} / m_{e}=206.7682843(52) \quad[25 \mathrm{ppb}] \\
\mu_{\mu} / \mu_{p}=3.183345107(84) \quad[26 \mathrm{ppb}]
\end{gathered}
$$
readout will provide the measurement of the downstrem-going, high energy, decay positrons.

\footnotetext{
${ }^{22}$ The corrections to the hyperfine splitting from hadronic vacuum polarization and $Z^{0}$ exchange are much smaller than the uncertainty arising from the mass ratio $m_{\mu} / m_{e}$.
} 


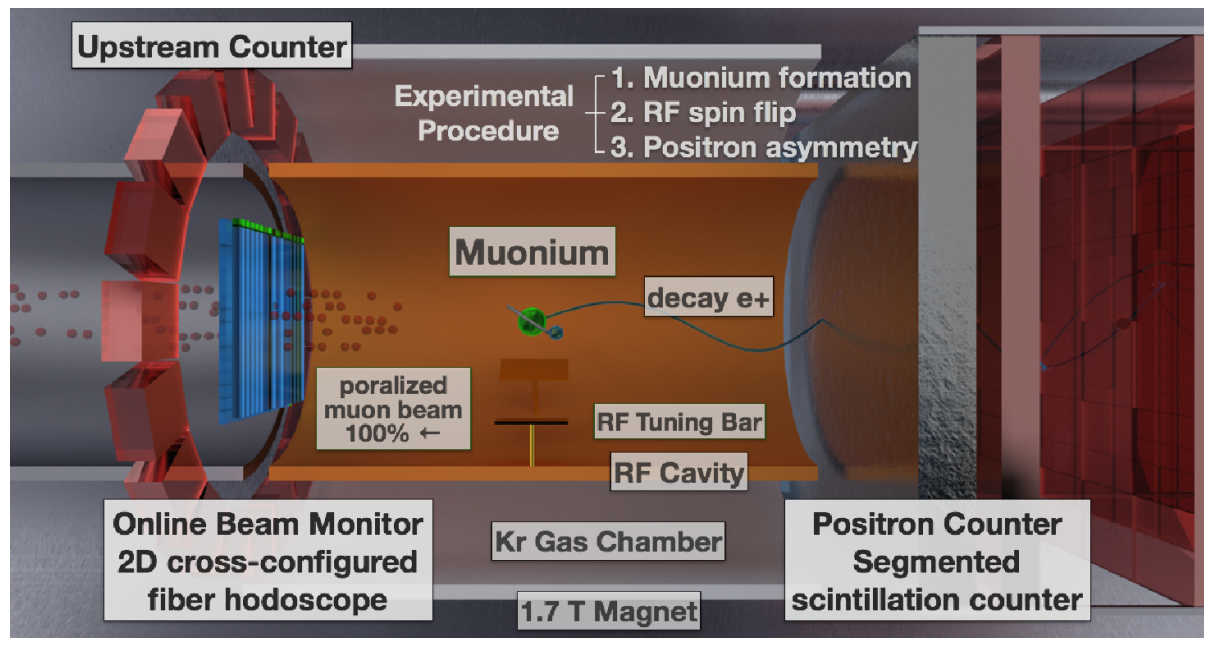

Figure 26: Schematic diagram of the J-PARC muonium experiment showing the pulsed muon beam, beam profile monitor, krypton gas chamber and microwave cavity, and the absorber and segmented detector for high energy positron detection. Figure courtesy MuSEUM collaboration.

During 2014 the MuSEUM collaboration have conducted tests of detector sub-systems and performed measurements of beam properties. The beginning of data taking is anticipated for 2015 .

\section{Muonic Lamb shift}

At first blush, one might wonder why we include a discussion of the proton charge radius in a muon physics review. Indeed, the inclusion makes sense here because it is a precision muon experiment that stirred things up with a result now known as the "Proton Radius Puzzle" [137. The CREMA collaboration at PSI reported a very precise measurement of the muonic hydrogen Lamb shift $L_{1 \mathrm{~S}}$; i.e., the $2 \mathrm{~S}_{1 / 2} \rightarrow 2 \mathrm{P}_{1 / 2}$ energy level difference in the $\mu p$ atom. The $\mathrm{S}$ energy levels are sensitive to the proton finite size, owing to their spherical symmetry and consequent overlap with the distributed charge distribution.

The motivation for the experiment was based on the need for a more precise determination of the proton charge radius $r_{p}$. Along with the Rydberg constant $R_{\infty}$, it is one of the two required inputs to calculate hydrogen energy levels using QED, where the S-state energy is given approximately by

$$
E(n S) \simeq \frac{R_{\infty}}{n^{2}}+\frac{L_{1 \mathrm{~S}}}{n^{3}},
$$

with $n$ the usual principal quantum number. The connection to $r_{p}$ can be obtained from the energy shift of an S-state level by

$$
\Delta E=\frac{2}{3} \pi \alpha\left|\Psi_{\mathrm{S}}(0)\right|^{2} r_{p}^{2}
$$


1620

with $\Psi_{\mathrm{S}}(0)$ the electron wavefunction at the origin. The experimental situation in hydrogen spectroscopy had achieved a precision great enough such that improved knowledge of $r_{p}$ in computing the finite-size effect was limiting [137, 138].

In muonic-hydrogen, the Bohr radius is $\sim 186$ times smaller than the corresponding one in ordinary hydrogen, which implies a greater overlap with the nucleus by a factor proportional to the cube of the radii, or $6.4 \times 10^{6}$. Thus, the sensitivity to the finite-size effect is greatly enhanced. The logical sequence to an improved QED test is as follows:

1. Measure the energy levels precisely in the $\mu p$ system.

2. Extract the proton charge radius $r_{p}$ from the shift in S-state levels relative to the essentially unaffected $\mathrm{P}$ states.

3. Use the improved knowledge of $r_{p}$ to better compare the measured $e p$ atomic energy levels to QED predictions.

At least, that was the idea.

However, the results of the $\mu p$ measurements indicated a $4 \%$ smaller charge radius, with a $0.6 \%$ uncertainty, compared to what had been commonly assumed. The standard methods had been low-energy $e-p$ scattering and ordinary hydrogen spectroscopy, where the finite size effect enters and one might assume QED to obtain the finite-size level shift, rather than test QED by externally knowing the finite size. The discrepancy between the muonic and electronic methods is significant - 7 standard deviations - such that any hope of simply using the independent $r_{p}$ extracted from muonic atoms is problematic. Consider the numeric values. The previous CODATA recommended value was $r_{p}=0.8775(51) \mathrm{fm}$ [4, while the muon result alone gives $r_{p}=$ $0.84087(39) \mathrm{fm}$ [139, 140]; note the 13 times smaller uncertainty in the muon measurement.

Besides the statistical significance, it has been generally agreed that the method employed in the muonic hydrogen measurement is nearly irrefutable; it involves laser spectroscopy with accurately calibrated absorption lines. The checking and double checking of the extrapolation of $r_{p}$ from $\Delta E_{2 \mathrm{~S}-2 \mathrm{P}}$ has revealed no error, or even much wiggle room of uncertainty. Perhaps the muon behaves differently than an electron. Perhaps it has a more complex sensitivity to the proton charge distribution, which would totally violate our earlier assertions of lepton universality. These ideas and other exotic suggestions have been discussed, and often dismissed or ruled out, in a vast literature that is summarized in Refs. 137, 138.

If there is no problem with the muon measurement, and not finding a credible exotic origin for the difference, then possibly there is a problem with both ordinary hydrogen spectroscopy and the low-energy elastic electron-proton scattering measurements. These latter two methods agree with one another on $r_{p}$, albeit with much larger error bars than the muonic Lamb shift measurement. Nevertheless, investigations have been raised about each method in searching for a resolution to the puzzle. 


\subsection{Proton radius from electron scattering and hydrogen spectroscopy}

While we refrain from a deep departure into $e-p$ scattering formalism, the

1665 Once confined, they can leave along the axis of the trap into a toroidal magnetic momentum filter and then onward to a target region that contains pure 


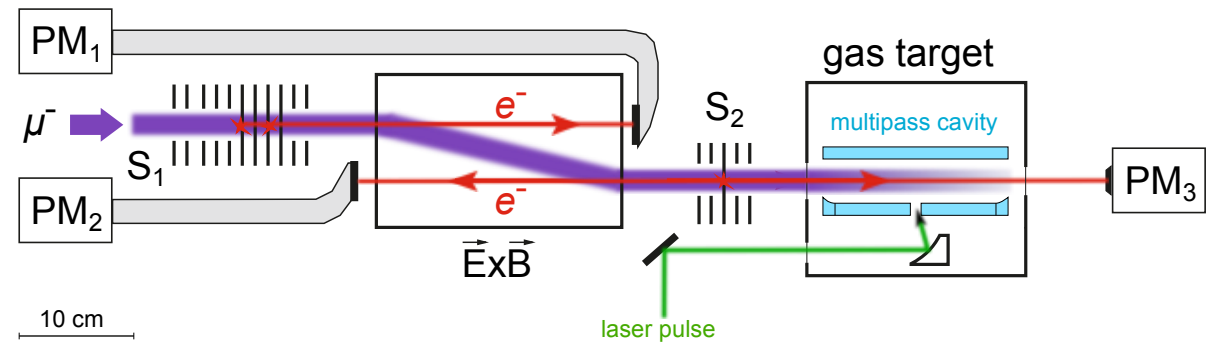

Figure 27: The CREMA experimental layout. The low-energy muon beam enters from the left and is detected by the emission of electrons in a thin set of carbon foils that then excite scintillators. The muon passes through a $\vec{E} \times \vec{B}$ velocity selector to separate it from the electrons. It then enters an ultra-low pressure hydrogen gas target. The PMT signals indicate the $t_{0}$ time and signal the laser to fire. The $5 \mathrm{~ns}$ long pulse is reflected in the multipass cavity giving an effective overlap time with the muonic atoms of about $75 \mathrm{~ns}$. If the delayed $2 \mathrm{P}$ state is formed, large-area avalanche photodiodes placed near the target are positioned to record the characteristic $1.9 \mathrm{keV}$ x rays. Figure courtesy R. Pohl.

hydrogen gas at low pressure, see Fig. 27. A muon entering the target will have passed through thin stacks of carbon foils. Low-energy electrons can be ejected, and subsequently detected in thin scintillators viewed by photomultiplier tubes. The signals so produced serve as a time marker of the arriving muon. The muon slowed in the process by normal $d E / d x$ energy loss has a probability to atomically capture in an excited $n$ level - typically 14 - and begin an ordinary cascade to the ground state. For optimized conditions of pressure, $\approx 1 \%$ of the atoms 1710 will result in a muon cascade terminating in the $2 \mathrm{~S}$ metastable state. This is the required starting point for a Lamb-shift measurement, and it was by no means an obvious or easy situation to prepare. For the CREMA conditions of a 1 mbar pressure hydrogen target, the metastable $2 \mathrm{~S}$ state will have a lifetime of about $1 \mu \mathrm{s}$ before undergoing collisional de-excitation [137. It is in that short window that the rest of the experiment must then work.

Once formed, the next challenge is to induce the $2 \mathrm{~S} \rightarrow 2 \mathrm{P}$ level transition by shining a properly tuned laser on the atoms. If the laser frequency corresponds to $\Delta E_{2 \mathrm{~S}-2 \mathrm{P}}$, the muon will transition to the appropriate $2 \mathrm{P}$ level, where it will then rapidly cascade to the $1 \mathrm{~S}$ level, emitting a characteristic $1.9 \mathrm{keV}$

${ }_{1720} \mathrm{x}$ ray. These four steps are shown in the left panel of Fig. 28. The idea of the experiment is to form the atom and then fire the laser $0.9 \mu \mathrm{s}$ later, simultaneously opening up a $75 \mathrm{~ns}$ wide gate to observe whether a $1.9 \mathrm{keV}$ x ray has been emitted. The laser frequency is tuned in discrete steps to scan the anticipated energy region around the expected $2 \mathrm{~S} \rightarrow 2 \mathrm{P}$ transition energy. The recorded 1725 number of $\mathrm{x}$ rays corresponding to the $2 \mathrm{P} \rightarrow 1 \mathrm{~S} \times$ ray vs. time will have two features. First, a large prompt peak will be present in $99 \%$ of the cases owing to the atom following a normal cascade to the $1 \mathrm{~S}$ ground state. A second, delayed peak, at $\sim 100$ times lower intensity will be present if and only if the laser frequency is correctly tuned to the $2 \mathrm{~S} \rightarrow 2 \mathrm{P}$ resonance; otherwise, the second peak ${ }_{1730}$ is absent. By repeating the experiment and slowly sweeping the laser frequency, 
a)

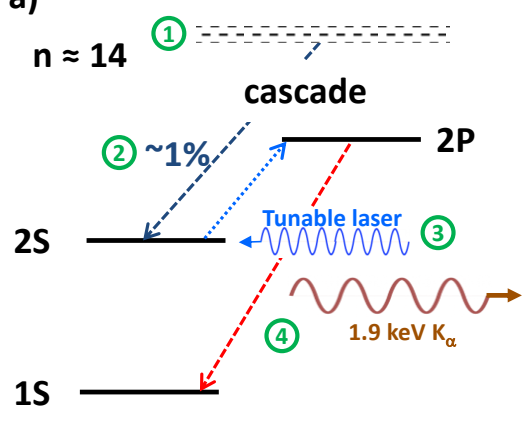

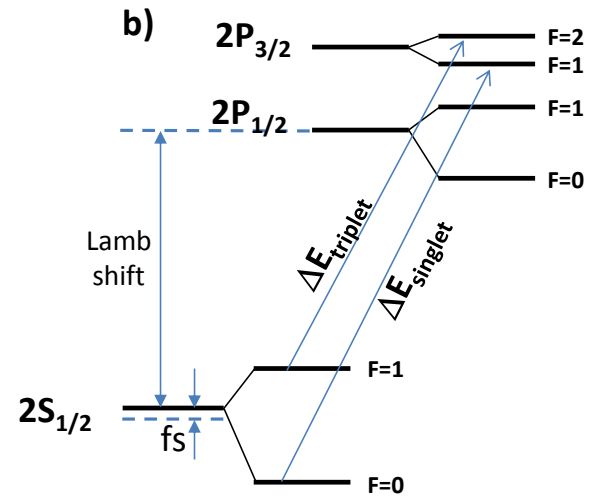

Figure 28: a) The four step schematic of how the experiment works. 1) The muon arrives in the target and captures in a high $n$ shell. 2) Muon cascade, with $\sim 1 \%$ stopping in the metastable $2 \mathrm{~S}$ state. 3) A tunable laser is fired $0.9 \mu$ s after each muon arrives in the target. 4) The $2 \mathrm{~S} \rightarrow 2 \mathrm{P}$ transition is induced if the laser frequency is on resonance, and the $2 \mathrm{P}$ state will decay rapidly to the $1 \mathrm{~S}$ ground state, emitting a $K_{\alpha}$ x ray that can be detected, serving to tag the process. b) Blowup of the level scheme showing the fine-structure splitting of the $\mathrm{P}$ levels and the hyperfine structure of $\mathrm{S}$ and $\mathrm{P}$ levels. The two measured and published transitions are indicated.

one obtains a very precise measurement of $\Delta E_{2 \mathrm{~S}-2 \mathrm{P}}$.

In practice, the $\mathrm{P}$ orbitals are split by atomic fine structure into the $\mathrm{P}_{1 / 2}$ and $\mathrm{P}_{3 / 2}$ levels, and both $\mathrm{S}$ and $\mathrm{P}$ levels are further split by the hyperfine interaction. The level scheme is shown on the right panel of Fig. 28, where the Lamb shift

1735 is defined as shown, and the effect of the finite-size ( $\mathrm{fs})$ correction on the $\mathrm{S}$ shell is highlighted. The two measured transition energies, $\Delta E_{T}$ (triplet) and $\Delta E_{S}$ (singlet) can be used in linear combinations that, together with known QED-based corrections not dependent on $r_{p}$, yield both the Lamb shift and the hyperfine splitting. In turn, one can not only deduce the discussed charge radius, but also the Zemach radius, $r_{Z}$, which is essentially a measure of the magnetic distribution inside the proton. While the extracted $r_{p}$ is significantly more precise than other methods, $r_{Z}$ is not. Its value of $r_{Z}=1.082(37) \mathrm{fm}$ is compatible with other methods and its uncertainty is many times larger. As quoted above, we obtain here from muon spectroscopy (MS): $r_{p}(M S)=$ $1745 \quad 0.84087(39) \mathrm{fm}[139,140$,

\subsection{Present and future work involving muons}

The CREMA collaboration has completed, but not yet published, additional Lamb-shift measurements in deuterium and helium. Three transitions in deuterium have been accurately measured, with preliminary interpretations that suggest consistency with the muonic hydrogen result; however, theoretical work continues so it is premature to draw firm conclusions. They have also completed Lamb-shift measurements on ${ }^{3} \mathrm{He}$ and ${ }^{4} \mathrm{He}$ systems in 2014, which are undergoing analysis. 
Suppose we do not find a ready solution. Then what? A proposal by the

\section{Nuclear muon capture}

\subsection{Basic features of muon capture}

Muon capture and beta decay are close cousins. Both processes

$$
\begin{array}{ll}
\text { 1. } \mu^{-}+[Z, A] \rightarrow[Z-1, A]+\nu_{\mu} \\
\text { 2. }[Z, A] \rightarrow[Z \pm 1, A]+e^{\mp}+\nu_{e}
\end{array}
$$

involve transmutations of protons into neutrons or vice-versa through a semileptonic weak interaction with a precisely-known leptonic current. However the energy release in the two reactions - set by the muon mass in the muon process and the nuclear mass difference in the beta process - are quite different. Con-

1780 sequently, the two processes can illuminate different features of the underlying weak nucleonic and nuclear interactions.

Muon capture occurs from the 1S ground state of a muonic atom; such atoms are formed when muons are stopped in matter. In light nuclei, where the overlap of the muon orbital with the nuclear volume is relatively small, the

1785 capture rate is small compared to muon decay. In heavier nuclei, where the overlap is much larger, the capture rate is large compared to muon decay ${ }_{23}^{23}$ For muonic hydrogen and muonic deuterium about $0.1 \%$ of muons undergo capture.

\footnotetext{
${ }^{23}$ The $\mathrm{Z}^{4}$-law for muon capture [146] states the capture rate is proportional to the fourth power of the effective charge $Z$ of the atomic nucleus.
} 
When muonic atoms are formed on non-zero spin nuclei $(I \neq 0)$ the $1 \mathrm{~S}$ ground state is split into two distinct hyperfine states with total angular momenta of $F=I+1 / 2$ and $F=I-1 / 2$. The possibility of muon capture from the singlet and triplet hyperfine states in hydrogen, and doublet and quartet hyperfine states in deuterium, is responsible for engendering capture on hydrogen isotopes with additional richness and additional complexity.

The first observation of muon capture on hydrogen was reported in 1962

1795 by Hildebrand [147] using a hydrogen bubble chamber. This experimenttogether with other early experiments using bubble chambers and liquid scintillator detectors - were important as evidence in support of the nascent $V-A$ theory of the weak interaction [20, 21]. Many other muon capture experiments have since been conducted.

$1800 \quad$ Muon capture on hydrogen isotopes offers a unique opportunity to determine elusive components of weak nuclear interactions - the induced pseudoscalar coupling of the proton and the two-body axial current of the deuteron. Herein we describe the recent progress in precision $\mu p$ and $\mu d$ experiments that address such elementary features of weak interactions.

\subsection{Muon capture on hydrogen, $\mu p \rightarrow n \nu$}

Muon capture is generally treated as a current-current weak interaction where the leptonic current and the nucleonic current have the familiar parity violating $V$-A structures. The leptonic current has the simple $\gamma_{\mu}\left(1-\gamma_{5}\right)$ form. The nucleonic current - because of its quark constituents and their strong interactions - is more complicated.

The most general form of the nucleonic $V$ - $A$ current is

$$
\begin{gathered}
+g_{v} \gamma^{\mu}+\frac{i g_{m}}{2 m_{N}} \sigma^{\mu \nu} q_{\nu}+\frac{g_{s}}{m_{\mu}} q^{\mu} \\
-g_{a} \gamma^{\mu} \gamma_{5}-\frac{g_{p}}{m_{\mu}} q^{\mu} \gamma_{5}-\frac{i g_{t}}{2 m_{N}} \sigma^{\mu \nu} q_{\nu} \gamma_{5}
\end{gathered}
$$

where $\gamma^{\mu}$ are the Dirac matrices, $q=p_{n}-p_{p}$ is the momentum transfer and $m_{\mu}$ and $m_{N}$ are the muon and nucleon mass. The nucleonic current contains six "coupling constants" that are functions of the momentum transfer-squared

$1815 q^{2}$. The couplings $g_{v}, g_{m}$, and $g_{s}$ are the vector, weak magnetism and induced scalar couplings of the hadronic vector current, $V$. The couplings $g_{a}, g_{p}$, and $g_{t}$ are the axial, induced pseudoscalar and induced tensor couplings of the hadronic axial current, $A$.

The terms involving $g_{v}, g_{a}, g_{m}$ and $g_{p}$ are called first-class currents while the terms involving $g_{s}$ and $g_{t}$ are called second-class currents 148. This distinction arises as first-class currents and second-class currents have opposite transformation properties under G-parity - an operation that links the transmutation of protons into neutrons with the transmutation of neutrons into protons. Consequently, the second-class contributions to the leading first-class currents only

1825 arise through G-parity breaking effects (e.g. the u-d quark mass difference and the electromagnetic corrections). No experimental evidence for second-class currents exists (for recent discussions see Ref. [149] regarding $g_{s}$ and Ref. 150] regarding $\left.g_{t}\right)$. 
In the conserved vector current hypothesis (CVC) 21] the weak vector current and isovector electromagnetic current are the components of a conserved vector-isovector current. The hypothesis relates the $g_{v}$ and $g_{m}$ terms of the weak vector current to the charge and magnetism terms of the isovector electromagnetic current. It predicts a "weak magnetism" analogous to magnetic effects in electromagnetic interactions and yields $g_{v}=1.0$ and $g_{m}=3.706$ at $q^{2}=0$. The roots of CVC were the closeness (1-2\%) of the constant $G_{F}$ determined in muon decay and the constant $G_{F} g_{v}$ determined in beta decay. This observation resembles the equality of the electric charge of the electron and the proton. Apparently, the bare weak vector charge of the proton, like the bare electric charge of the proton, is protected from renormalization via emission 1840 and absorption of virtual pions, by a conservation law 151. Nowadays, the conserved vector-isovector current is an integral part of our understanding of the nucleon's quark structure.

Concerning the axial current, the axial coupling is well-determined from measurements of neutron beta decay that yield $g_{a}=1.2723 \pm 0.0023$ 43. In contrast with $g_{v}$ the value of $g_{a}$ is (slightly) modified by strong interactions and we speak of a partially conserved axial current in place of an exactly conserved vector current. This partially conserved axial current reflects an underlying approximately conserved chiral symmetry of strong interactions [152].

The remaining term is the induced pseudoscalar coupling $g_{p}$; an interaction that plays a significant role in muon capture but not in beta decay. For fifty years the value of $g_{p}$ has been uncertain and the predictions for $g_{p}$ have been untested. The interest in $g_{p}$ stems from more than just its status as the poorlyknown piece of the weak nucleonic current. A precise value [153]

$$
g_{p}=8.44 \pm 0.23
$$

is predicted by quantum chromodynamics - a prediction that is closely connected to spontaneous symmetry breaking in low energy QCD and the dynamical origins of the hadronic masses.

To understand the low-energy realization of chiral symmetry it is helpful to consider the hypothetical limit of massless $\mathrm{u}$ and d quarks. For $m_{u}=m_{d}=0$ QCD possess an exact $\mathrm{SU}(2)_{L} \times \mathrm{SU}(2)_{R}$ chiral symmetry, i.e., there are sepa-

1855 rate copies of isospin symmetry for the left-handed quarks and the right-handed quarks. This symmetry generates two conserved currents, a vector current corresponding to the sum of left- and right-handed quark currents and a conserved axial current corresponding to the difference of left- and right-handed quark currents. At low energies this chiral symmetry is spontaneously broken through QCD interactions. As a result the hadrons acquire mass and the pion appears as the Goldstone boson of the broken symmetry. Still the underlying currents remain conserved currents and thereby dictate a precise relation between $g_{a}$ and $g_{p}$.

Of course, up and down quarks are not exactly massless and consequently chiral symmetry and axial current conservation are also not exact. This small explicit breaking of chiral symmetry modifies the relation between $g_{a}$ and $g_{p}$ 


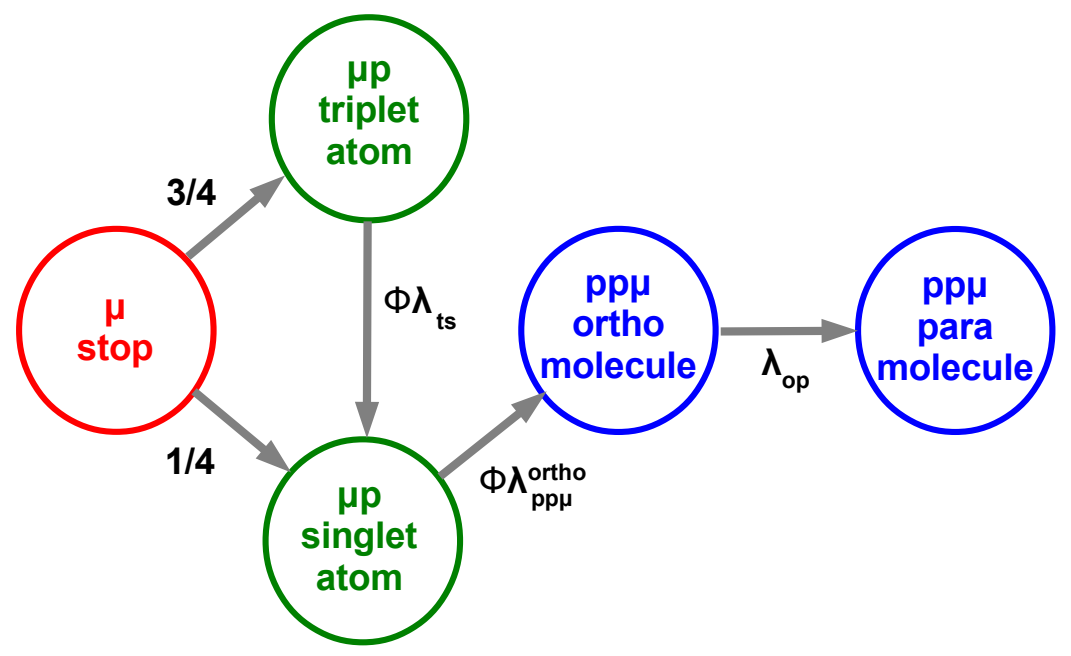

Figure 29: Schematic diagram showing the important atomic and molecular states and transition rates for muon stops in isotopically pure hydrogen. The $\mu p$ atoms are initially formed in a statistical mix of triplet atoms (3/4) and singlet atoms $(1 / 4) . \phi \lambda_{t s}$ is the densitydependent triplet-to-singlet transition rate, $\phi \lambda_{p p \mu}^{\text {ortho }}$ is the density-dependent ortho-molecular formation rate, and $\lambda_{o p}$ is the density-independent ortho-to-para transition rate.

but still - through older current algebra techniques or newer chiral perturbation theory - a precise prediction for $g_{p}$ results. The value of $g_{p}$ is thereby tied to our modern understanding of the strong interaction that incorporates its 1870 approximate chiral symmetry and partial axial current conservation as well as the dynamical origins of the hadronic masses (for further details see Ref. 154, 155).

\subsubsection{Muon chemistry in pure hydrogen}

Although the theoretical relation between the $\mu p \rightarrow n \nu$ capture rate and the weak coupling constants is quite straightforward - a complication exists. The $\mu p$ atoms that form when muons are stopped in hydrogen are small and neutral. Consequently, they scatter off and react with the surrounding $\mathrm{H}_{2}$ molecules, thus causing the $F=0,1$ hyperfine populations to evolve with time. This evolution depends on the thermalization of the 'hot' $\mu p$ atoms in the $\mathrm{H}_{2}$ environment 1880 as well as chemical reactions that form muonic molecules (the bound-states of a single negative muon and two hydrogen nuclei). A detailed knowledge of the relevant atomic and molecular processes - as shown in Fig. 29 -is therefore needed to extract $g_{p}$ from experimental data.

The $\mu \mathrm{p}$ atom is initially formed in an excited state with a principal quantum 
number $n \sim 14$. The excited atom rapidly de-excites through combinations of Auger emission, radiative decays and Coulomb de-excitation. On reaching the $1 \mathrm{~S}$ ground state the $\mu p$-atoms have kinetic energies of typically $1 \mathrm{eV}$ (for details see [156]) and a statistical population of the hyperfine states (3/4 triplet atoms and $1 / 4$ singlet atoms).

These energetic atoms are rapidly thermalized by elastic and spin-flip collisions with the atomic nuclei of the surrounding $\mathrm{H}_{2}$ molecules. When their energies fall below the $0.18 \mathrm{eV} \mu p$ hyperfine splitting, the singlet-to-triplet transitions are energetically forbidden and triplet-to-singlet transitions depopulate the higher-lying triplet state. The triplet lifetime is about $0.1 \mathrm{~ns}$ in liquid $\mathrm{H}_{2}$ and about 10 ns in 10 bar $\mathrm{H}_{2}$ gas.

At sufficient densities $p p \mu$ molecules form. Like ordinary $\mathrm{H}_{2}$ molecules, there exists both para $\mu$-molecular hydrogen with a total nuclear spin $I=0$ and ortho $\mu$-molecular hydrogen with a total nuclear spin $I=1$. The para molecule is the true ground state of the $p p \mu$ molecule. Importantly, the two molecules have different $\mu p$-spin decompositions; the para-molecule being 3:1 triplet-to-singlet and the ortho-molecule being 1:3 triplet-to-singlet.

The $p p \mu$ molecules are formed by collisions between $\mu p$ atoms and surrounding $\mathrm{H}_{2}$ molecules via Auger emission $\mu p+H \rightarrow p p \mu+e$. The calculated rate of the E1 Auger transition forming ortho-molecules $\lambda_{p p \mu}^{\text {ortho }} \simeq \phi \times 1.8 \times 10^{6} \mathrm{~s}^{-1}$ is much faster than the E0 Auger transition forming para-molecules $\lambda_{p p \mu}^{p a r a} \simeq$ $\phi \times 0.75 \times 10^{4} s^{-1}$ ( $\phi$ is the $\mathrm{H}_{2}$ density normalized to the liquid $\mathrm{H}_{2}$ density $\phi_{o}=4.25 \times 10622$ atoms $\left./ \mathrm{cm}^{3}\right)$. A recent measurement [157] of the total rate of molecular formation found $\lambda_{p \mu p}=\phi \times 2.01 \pm 0.07 \times 10^{6} s^{-1}$ in reasonable agreement with theoretical predictions.

Naively, the $\Delta I=0$ selection rule for E1 transitions forbids the decay of ortho molecules to para molecules. However, as recognized by Weinberg, through relativistic effects that mix ortho- and para-states the ortho $p p \mu$ molecules do gradually decay into para $p p \mu$ molecules. The decay rate was computed by Bakalov et al. [158] to be $\lambda_{o p}=7.1 \pm 1.2 \times 10^{4} \mathrm{~s}^{-1}$ (this rate is indepen-

1915 dent of density). Unfortunately, the two published measurements for $\lambda_{o p}$ of $(4.1 \pm 1.4) \times 10^{4} s^{-1}\left[159\right.$ and $(10.4 \pm 1.4) \times 10^{4} s^{-1}$ [160], are in significant disagreement.

\subsubsection{Experimental approaches to $\mu$ capture}

The "neutron approach" to studying $\mu p \rightarrow n \nu$ capture involves stopping muons in hydrogen and detecting the resulting $5.2 \mathrm{MeV}$ capture neutrons. Such experiments were conducted in liquid hydrogen and gaseous hydrogen and achieved precisions of roughly $10 \%$ in the effective capture rate for the relevant $F=0,1$ populations. The neutron method was, however, limited by the necessary determination of the neutron detection efficiencies.

The "lifetime approach" to studying $\mu p \rightarrow n \nu$ capture avoids directly detecting the reaction products of muon capture. Rather, it determines the capture rate $\Lambda$ from the difference between the disappearance rates of the $\mu p$ atom and 
the positive muon, i.e ${ }^{24}$

$$
\Lambda=\lambda_{\mu p}-\lambda_{\mu^{+}}
$$

where $\lambda_{\mu p} \equiv 1 / \tau_{\mu p}$ and $\lambda_{\mu^{+}} \equiv 1 / \tau_{\mu^{+}}$are obtained from the measured time distributions of the decay electrons and positrons, respectively. The experiment is difficult as the decay rate is roughly 1000 times the capture rate and therefore the two disappearance rates are very similar - thus requiring extraordinarily precise $\mu p$ and $\mu^{+}$lifetime measurements. The lifetime approach was pioneered by Bardin et al. [161, at Saclay.

A serious concern for both approaches was muon stops in $Z>1$ surrounding materials. The detection of capture neutrons or decay electrons from muon stops in surrounding materials would alter the time distribution and distort the measured lifetime. Therefore the target vessel, etc., were typically constructed from high-Z materials so stopping muons were rapidly absorbed. Similarly, the small size and neutrality of $\mu p$ atoms exposes the muon to transfer to any $Z>1$ contaminants in the $\mathrm{H}_{2}$ gas. Again detection of capture neutrons or decay electrons from gas contaminants would distort the measured lifetime.

\subsubsection{MuCap experiment}

The MuCap experiment 34 was conducted at PSI. It used a custom-built, muon-on-demand beam to increase the sample of decay electrons while mitigating the effects of muon pileup. It also used an active target to verify the stopping of muons in hydrogen and monitor the effects of gas impurities.

The experiment was performed in $\mathrm{H}_{2}$ gas of high chemical and isotopic purity at 10 bar pressure and room temperature. Under these conditions the triplet atoms are short-lived and the $p p \mu$ molecules are rarely formed - thus preparing a nearly-pure sample of singlet atoms and enabling an unambiguous measurement of the $\mu p$ singlet capture rate $\Lambda_{S}$.

The experimental setup is shown in Fig. 30. A series of incoming muon detectors that consisted of a plastic scintillator and a planar multiwire chamber together determined the arrival time and provided the pile-up protection of beam muons. A series of outgoing electron detectors that consisted of consecutive layers of cylindrical multiwire chambers and segmented plastic scintillators together determined the times and trajectories of the decay electron. On iden-

1955 tifying a muon the upstream electrostatic kicker was turned on and thereby the muon beam was turned off.

The incoming muons were stopped in a hydrogen-filled time projection chamber (TPC). The TPC comprised a 5.04 liter active volume with a vertical drift field and a horizontal readout plane of perpendicular anode wires and cathode strips. The analog signals from anode wires and cathode strips were fed to three discriminator thresholds that triggered on: incoming muons (denoted $E L$ ), the Bragg peak of stopping muons (denoted $E H$ ), and the high ionization

\footnotetext{
${ }^{24}$ For $\mu p$ atoms the disappearance rate $\lambda_{\mu p}$ is the sum of the $\mu$ decay rate and the $\mu$ capture rate whereas for positive muons the disappearance rate is simply the $\mu$ decay rate.
} 


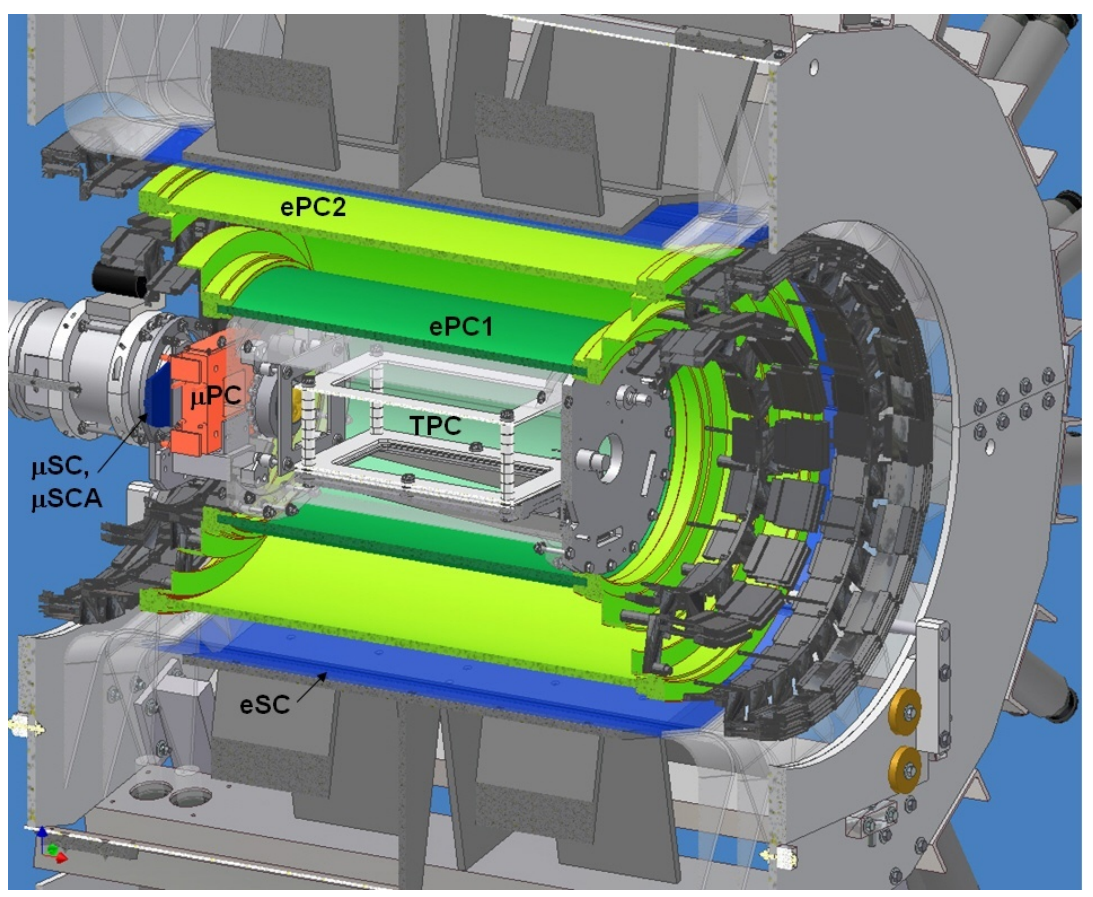

Figure 30: Cutaway diagram of the MuCap experiment indicating the muon counters ( $\mu \mathrm{SC}$, $\mu \mathrm{PC}), \mathrm{H}_{2}$ time projection chamber (TPC), and electron counters (ePC1, ePC2, eSC). The muon counters determine the muon arrival time, the electron counters determine the muon decay time, and the $\mathrm{H}_{2}$ TPC validates the muon stopped $\mathrm{H}_{2}$ gas. 
of charged products from muon capture on gas impurities (denoted $E V H$ ). The discriminator hits were recorded by multihit TDCs.

The experiment employed custom isotope separation and gas purification units. Isotopically pure $\mathrm{H}_{2}$ gas was prepared from commercial, isotopicallypure hydrogen by repeated cycles of fractional distillation. Chemically pure $\mathrm{H}_{2}$ gas was maintained by recycling the gas through a purification system that incorporated a cold trap and micro-porous filters. The experiment achieved a deuterium contamination of $<10 \mathrm{ppb}$ and a water contamination of about $9 \mathrm{ppb}$.

A crystal oscillator was used for the timebase of the readout electronics. The collaboration was blinded to the exact frequency of the timebase during the data taking and the data analysis. Only after completing all the analyses was the frequency revealed.

The experiment accumulated about $1.2 \times 10^{10}$ negative muon decays from pure $\mathrm{H}_{2}$ gas, $0.6 \times 10^{10}$ positive muon decays from pure $\mathrm{H}_{2}$ gas, as well as decay electrons from impurity-doped gas that permitted the investigation of muon transfer to gas impurities and formation rates of muonic molecules.

The decay curves were constructed from the measured time difference $\left(t_{e}-t_{\mu}\right)$ between an incoming muon signal in the muon scintillator and an outgoing electron signal in the electron scintillators. The TPC was used to validate that the muon had stopped in $\mathrm{H}_{2}$ gas. The TPC data showed stopping muons as a trail of $E L$ hits that led to several $E H$ hits at the stop location. The algorithm for authenticating a stop was optimized to handle the effects of (i) the hard scattering of incident muons from target protons into surrounding materials, and (ii) the possible interference between the incoming muon ionization and the outgoing electron ionization in the TPC.

The experiment also pioneered the in-situ measurement of gas impurities with the TPC. Muon transfer to gas impurities and subsequent capture on $Z>1$ nuclei was identified by single, delayed $E V H$ hits at the stopping location. From the measured rates and time distributions of muon stops with subsequent $E V H$ hits the necessary corrections due to $\mathrm{N}_{2} / \mathrm{H}_{2} \mathrm{O}$ impurities in the pure $\mathrm{H}_{2}$ gas were then derived.

The measured decay curves were fit to determine the muonic hydrogen lifetime. In principle - due to the muon kinetics and the time evolution of the $\mu p$ spin-states - the theoretical time distribution is not exactly a single exponential decay curve. However, in practice a single exponential was a good fit to the time distribution and adequately determined the muon disappearance rate.

Two corrections were necessary to extract the singlet capture rate $\Lambda_{S}$ from the difference $\lambda_{\mu p}-\lambda_{\mu^{+}}$between the $\mu^{ \pm}$disappearance rates. One correction accounted for the small population of $p p \mu$ molecules with singlet atoms. Another correction accounted for the slight difference in the decay rate of a bound muon versus a free muon. After these corrections of about $18 \mathrm{~s}^{-1}$ and $12 \mathrm{~s}^{-1}$ respectively the final result of

$$
\Lambda_{S}=715.6 \pm 5.4(\text { stat }) \pm 5.1(\text { syst }) s^{-1}
$$

was obtained [157]. Unlike many earlier experiments, the MuCap result is essentially free from ambiguities associated with muonic molecule formation. 


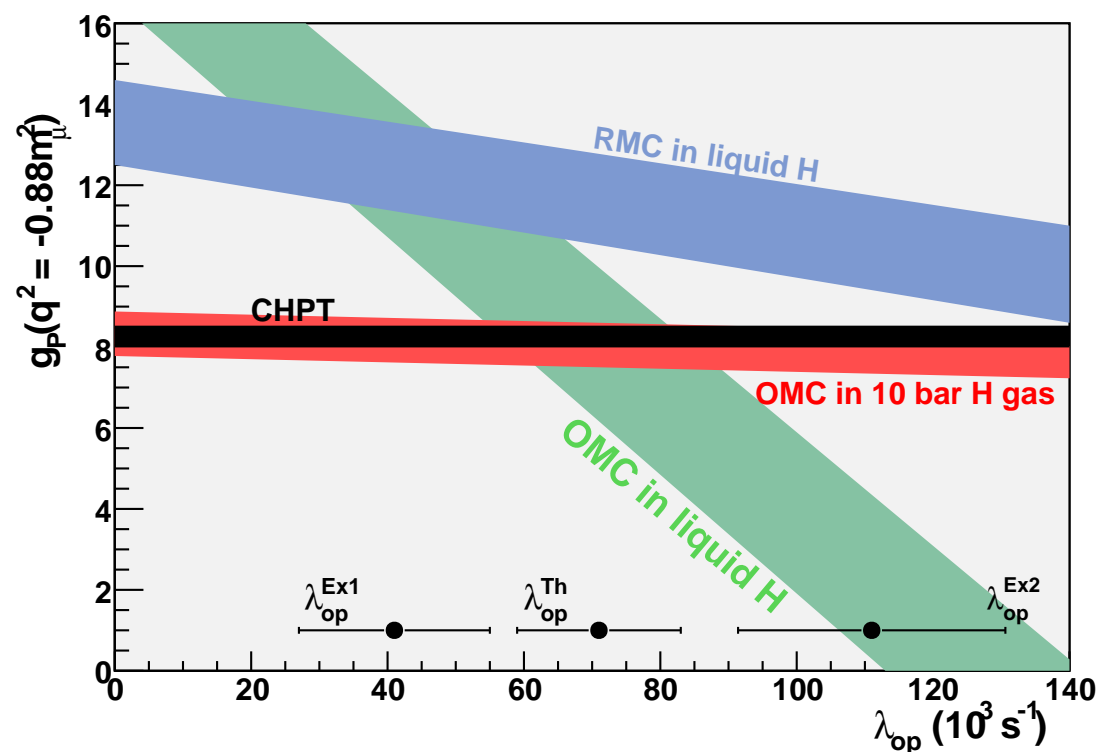

Figure 31: The induced pseudoscalar coupling $g_{p}$ versus the ortho-to-para molecular transition rate $\lambda_{o p}$. It shows the recent result of the MuCap experiment derived from ordinary muon capture in 10 bar gas and earlier results of Ref. [161] for ordinary muon capture in liquid hydrogen and Ref. 162 for radiative muon capture in liquid hydrogen. The data points indicate the experimental results [159, 160] and the theoretical calculation [158] of the orthoto-para transition rate. The MuCap result from $10 \mathrm{bar}_{2}$ gas is essentially free of ambiguities associated with $\lambda_{o p}$. 
Using the latest theoretical calculations of $\mu p \rightarrow n \nu$ capture [153] and incorporating radiative corrections [163, the MuCap measurement of singlet capture thereby determines the coupling $g_{p}$. Using $g_{a}=1.2701 \pm 0.0025$ for the axial coupling [164, the MuCap result for $\Lambda_{S}$ implies a value of

$$
g_{p}=8.06 \pm 0.48 \pm 0.28
$$

for the induced pseudoscalar coupling (the uncertainties are associated with the MuCap measurement and the $\chi \mathrm{PT}$ calculation of the capture rate, respectively).

The MuCap result for $g_{p}$ is in good agreement with the original predictions of current algebra and the modern predictions of chiral perturbation theory (Eqn.

2005 41). As shown in Fig. 31 the result for $g_{p}$ is also essentially free from the ambiquities associated with the limited knowledge of the ortho-to-para molecular transition rate that afflicted earlier experiments in liquid hydrogen. The result verifies our modern understanding of approximate chiral symmetry and partial axial current conservation in QCD. Such concepts are the foundations of our 2010 understanding of the origins of the hadron masses and the pion's significance as the Goldstone boson of a broken symmetry.

\subsection{Muon capture on deuterium, $\mu d \rightarrow$ nnv}

At a basic level the atomic nucleus is more than just an assembly of neutrons and protons. It incorporates such non-nucleonic degrees-of-freedoms as virtual pions and delta particles. With $g_{v}, g_{m}, g_{a}$, and $g_{p}$ all well-measured, the weak interaction offers a precise probe for exploring such exotic constituents of nuclear matter.

Muon capture on deuterium is nature's bridge between weak nucleonic and nuclear interactions. As such it parallels the role of radiative capture $n p \rightarrow d \gamma$

2020 on hydrogen and photo-disintegration $\gamma d \rightarrow n p$ of deuterium for electromagnetic processes. The $n p \rightarrow d \gamma$ reaction provided the first unequivocal evidence for non-nucleonic degrees-of-freedom in electromagnetic interactions. These nonnucleonic effects were surprisingly large with pion currents contributing roughly $10 \%$ of thermal neutron capture.

The interest in exchange currents in weak interactions is more than theoretical. The $\mu d \rightarrow n n \nu$ reaction is closely related to other $A=2$ weak processes including $p p \rightarrow d e \nu$ thermonuclear fusion in stars and $\nu$ d interactions in heavywater neutrino detectors. It represents the only $A=2$ weak interaction that is measurable and calculable to high precision. As such, the reaction is crucial to quantitatively understanding the non-nucleonic contributions to weak nuclear interactions and their influence on such processes as big-bang nucleosynthesis and stellar evolution as well as ordinary and double $\beta$-decay.

The $\mu d \rightarrow n n \nu$ process is dominantly an allowed Gamow-Teller transition from the ${ }^{3} \mathrm{~S}_{1}$ deuteron ground state to the ${ }^{1} \mathrm{~S}_{1} n n$ continuum state. Due to the $2035 \quad V$ - $A$ structure of the weak interaction the deuterium capture rate from doublet $(F=1 / 2)$ atoms is much larger than quartet $(F=3 / 2)$ atoms. Given our excellent knowledge of the nucleon weak couplings and the deuteron nucleonic 
wavefunction, the major uncertainty in $\mu d$ capture is the poorly-known contribution of the two-body axial current. A precision measurement of $\mu d$ capture can resolve this two-body current and thereby advance our theoretical understanding of many weak nuclear processes.

Until fairly recently the theoretical work on $\mu$ d capture was based on phenomenological potential models of nucleon-nucleon interactions. Using phenomenological potentials, sophisticated calculations that incorporated detailed

2045 initial- and final-state nucleonic wavefunctions augmented by simplified models of non-nucleonic contributions were performed by Tatara et al. [165, Doi et al. [166] and Adam et al. [167. These calculations gave rates $\Lambda_{D}$ for doublet capture of typically 390-400 $\mathrm{s}^{-1}$. The calculations suggested a two-body axial contribution of roughly $5 \%$ that mostly originates from delta excitation via pion exchange between nucleons.

Chiral effective field theory $(\chi \mathrm{EFT})$ was first developed for systems of pions, then extended to systems involving a single nucleon, and eventually applied to few-body nuclear systems. Its development has profoundly altered the theoretical treatment of weak interactions on few-body nuclei. $\chi$ EFT established

2055 a rigorous, unified framework for calculations that obeys the underlying symmetries of quantum chromodynamics while utilizing the pions and nucleons as low-energy degrees-of-freedom. It is based on a systematic expansion in small parameters - the momentum transfer, pion mass and nuclear binding energy where leading-order terms are computed and higher-order terms are neglected.

2060 Each of the calculated terms involves a low-energy constant that must be determined from data.

In $\chi$ EFT, a single low energy constant determines the two-body axial current contribution in weak nuclear processes (this low energy constant is denoted as $\hat{\mathrm{d}}_{R}$ or $\mathrm{L}_{1 A}$ in different versions of effective theories). The $\mu d \rightarrow n n \nu$ process offers an unmatched opportunity for determining this constant to a precision comparable to the recent caculations.

Over recent years a number of calculations of $\Lambda_{D}$ have been performed with increasing sophistication in the EFT framework. The two most recent calculations, which consistently treat the nuclear wavefunctions and the weak opera-

2070 tors, were conducted by Marcucci et al. [168, yielding $399 \pm 3 \mathrm{~s}^{-1}$, and by Adam et al. [169, yielding 383.8-392.4 $\mathrm{s}^{-1}$.

\subsubsection{Muon chemistry in pure deuterium}

Just as atomic and molecular processes can complicate the interpretation of $\mu p$ capture data, such atomic and molecular processes also complicate the interpretation of $\mu d$ capture data. A detailed knowledge of relevant atomic and molecular processes - as shown in Fig. 32 -is therefore required.

The $\mu d$ atoms are formed in excited states that rapidly de-excite to the 1S ground state by Auger emission, radiative decays and Coulomb collisions, thus yielding a statistical mix of "hot" doublet and quartet atoms. In many 2080 respects the chemical reactions of $\mu d$ atoms are very similar to $\mu p$ atoms. Both $\mu d$ and $\mu p$ are tiny, neutral atoms that easily penetrate the electronic clouds of surrounding molecules to scatter off and react with atomic nuclei. Like $\mu p$ 


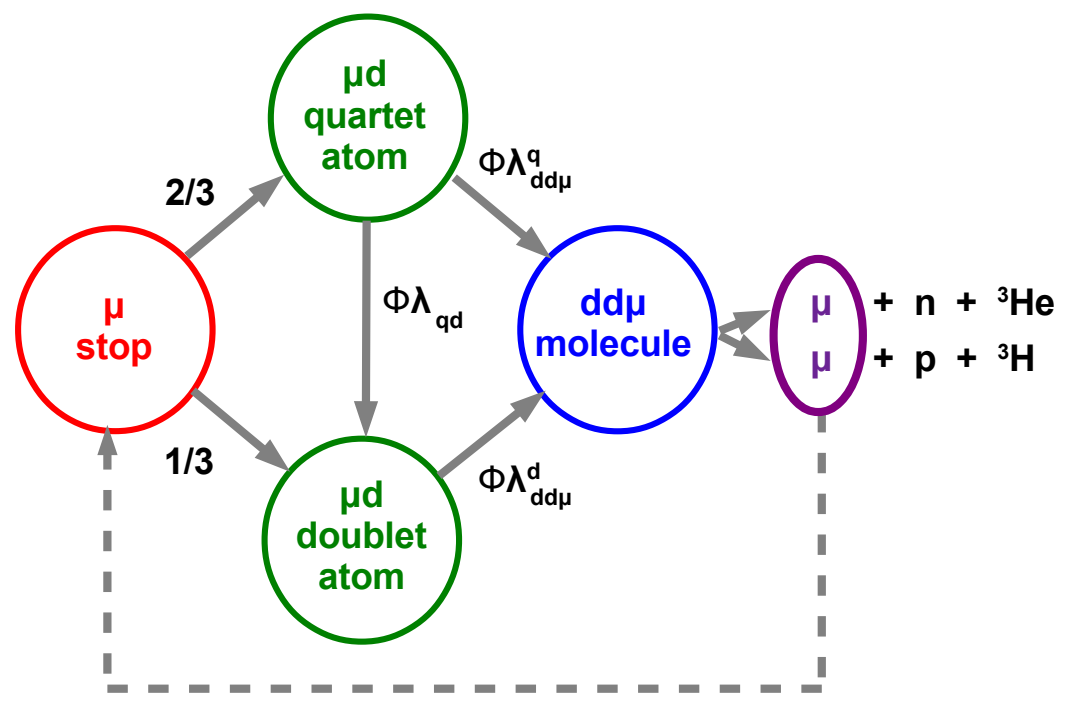

Figure 32: Schematic diagram showing the important atomic and molecular states and transition rates for muon stops in isotopically pure deuterium. The $\mu p$ atoms are initially formed in a statistical mix of quartet atoms (2/3) and doublet atoms (1/3). $\phi \lambda_{q d}$ is the density-dependent quartet-to-doublet transition rate and $\phi \lambda_{d d \mu}^{q}$ and $\phi \lambda_{d d \mu}^{d}$ are the densitydependent molecular formation rates from the quartet and doublet states. Also shown is the muon recycling following $d d \mu$ molecule formation and $\mu$-catalyzed fusion. 
atoms, the $\mu d$ atoms are thermalized by elastic and spin-flip collisions with surrounding nuclei. When the $\mu d$ energy falls below the $0.043 \mathrm{eV}$ hyperfine splitting, the spin-flip collisions then depopulate the quartet atoms in favor of doublet atoms. However, the cross sections are considerably smaller for $\mu d+d$ scattering than $\mu p+p$ scattering and consequently the quartet $\mu d$ atoms in $D_{2}$ are longer-lived than triplet $\mu p$ atoms in $\mathrm{H}_{2}$ (for details see [170]).

One new feature of $\mu d$ chemistry is temperature-dependent resonant formation of $d d \mu$ molecules - a process by which the $d d \mu$ binding energy is absorbed by $\mathrm{D}_{2}$ vibro-rotational modes. For example, at cryogenic temperatures, while $d d \mu$ formation by doublet $\mu d$ atoms involves a rather slow, non-resonant process, the $d d \mu$ formation by quartet $\mu d$ atoms involves a fast, resonant process.

Another new feature of $\mu d$ chemistry is muon catalyzed fusion [171. The possibility of muon catalysis of nuclear reactions was first proposed by Frank 172 in 1947 and later considered by Gerstein, Sakharov and Zeldovich in the early 1950 s as a possible energy source. Its first observation was entirely accidentalAlvarev et al. [173] identifying the puzzling tracks following muon stops in bubble chambers as muons released following catalyzed fusion.

This release of muons from $d d \mu$ molecules is an additional dimension of $\mu d$ chemistry. In $d d \mu$ molecules the fusion reactions are

$$
\begin{aligned}
& \text { 1. } d d \rightarrow n{ }^{3} \mathrm{He} \\
& \text { 2. } \quad d d \rightarrow p^{3} \mathrm{H},
\end{aligned}
$$

the former yielding an intense source of mono-energetic neutrons in $\mu d$ experiments. On forming a $d d \mu$ molecule, the fusion reaction occurs essentially instantaneously. The reactions generally release muons but on occasion they will stick to the charged products of the fusion reaction.

\subsubsection{MuSun experiment}

2105 The $\mu$ d doublet capture in pure deuterium was previously measured using the lifetime technique and a liquid $\mathrm{D}_{2}$ target yielding $\Lambda_{d}=470 \pm 29 \mathrm{~s}^{-1}$ [174] and using the neutron technique and a gaseous $D_{2}$ target yielding $\Lambda_{d}=409 \pm 40 \mathrm{~s}^{-1}$ [175. These experiments were conducted more than twenty five years ago.

The MuSun experiment 38 is using the lifetime technique to measure the $\mu d$ doublet capture rate $\Lambda_{D}$ to about $1.5 \%$ and thereby improve by roughly five-fold the current knowledge of two-body axial current contributions to $A=2$ weak nuclear processes. The approach requires the preparation of a nearly-pure sample of doublet atoms, a $10 \mathrm{ppm}$ measurement of the $\mu d$ atom lifetime, and careful monitoring of isotopic and chemical impurities in deuterium. The experiment builds on the development and the innovations in the MuCap experiment.

As shown in Fig. 33, the MuSun experiment is using a novel cryogenic $\mathrm{D}_{2}$ time projection chamber. The temperature of $34 \mathrm{~K}$ and pressure of 5-6 bar were chosen to prepare an optimal population of nearly-pure doublet atoms. At this temperature and pressure the quartet atoms rather quickly decay to dou2120 blet atoms but regeneration of quartet atoms by muon recycling following $d d \mu$ molecule formation and $d d$-fusion is quite small. The time projection chamber 


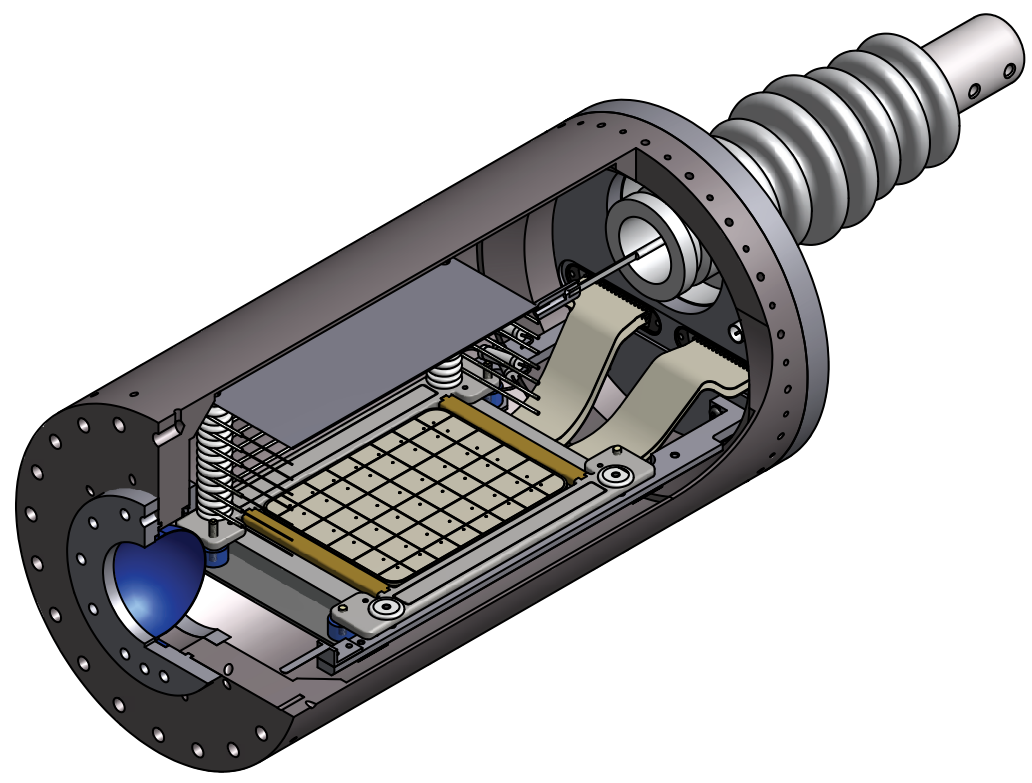

Figure 33: Cutaway diagram of the MuSun low temperature, high pressure, $\mathrm{D}_{2}$ time projection chamber. It shows the cryogenic pressure vessel, the beryllium beam entrance window and liquid neon cooling system, as well as the horizontal cathode plane, $6 \times 8$ segmented anode plane and field-shaping wires.

also provides the stop definition for incoming muons and monitoring of $\mu d$ kinetics including both the formation of muonic molecules and the transfer to gas impurities.

The MuSun setup consists of an incoming muon counter package, outgoing electron counter package, the cryogenic $\mathrm{D}_{2}$ time projection chamber and a liquid scintillator neutron detector array. The muon and electron counter packages are conventional arrangements of plastic scintillators and proportional chambers that were originally constructed for the MuCap experiment.

The high pressure, low temperature, $\mathrm{D}_{2}$ TPC works as follows. Ionization is collected via a vertical drift field and a $6 \times 8$ segmented, horizontal anode plane, then readout via cryogenic pre-amplifiers and 8-bit, $25 \mathrm{MHz}$ waveform digitizers. The TPC and associated electronics were designed for good energy resolution $(\sim 10 \mathrm{keV})$ to thereby enable the clean identifcation of fusion products and muon capture on gas contaminants.

As in MuCap the muon and electron plastic scintillators determine the time interval between the incoming muon and the outgoing electron in order to construct the decay curve and extract the $\mu d$ lifetime. A stop definition that is derived from the signals in the anode pads is used to validate the entries in the 2140 time distribution.

Processes that involve either muon transfer to chemical impurities or molec- 


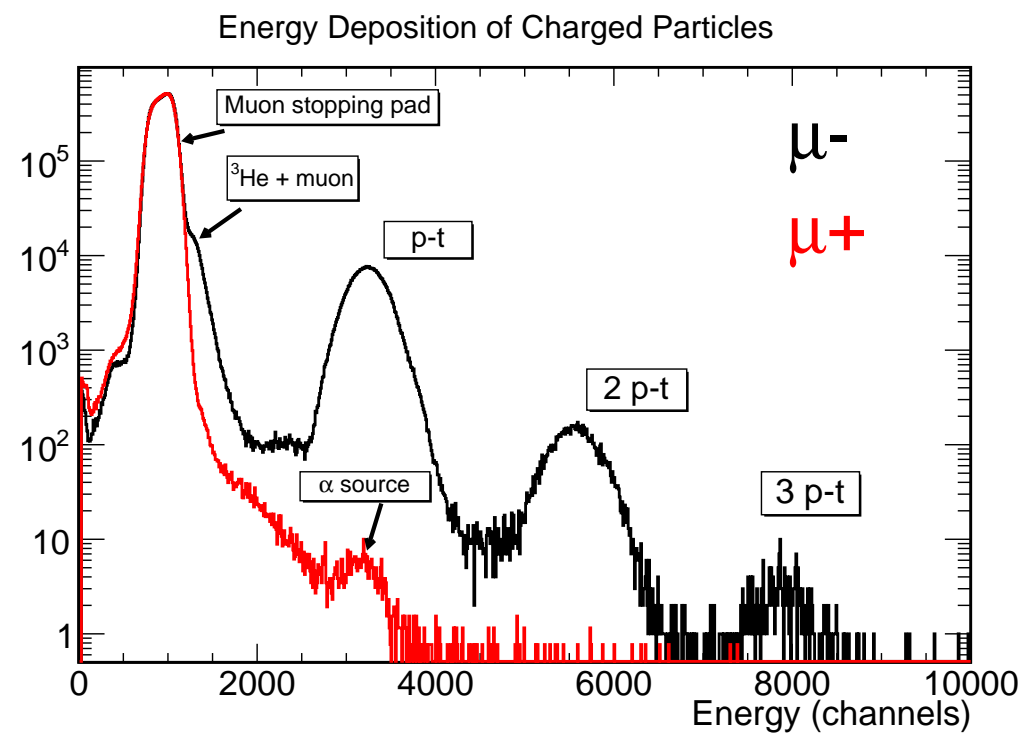

Figure 34: Measured energy deposition of stopping negative and positive muons in the MuSun cryogenic TPC. The energy is defined as the summed signal from the muon stopping pad and its eight neighboring pads. The positive muon energy distribution shows a large peak that corresponds to the stopping muons and a small peak that corresponds to an in-situ alpha calibration source. The negative muon energy distribution additionally shows the effects of muon-catalyzed fusion and the additional energy deposition associated with the chargedparticle products of the ${ }^{3} \mathrm{He}$ and $\mathrm{p}{ }^{3} \mathrm{H}$ fusion channels. In the particular case of the $\mathrm{p}^{3} \mathrm{H}$ channel, the occurrence of one, two and three fusions following a single muon stop are clearly discernable.

ular formation on isotopic impurities impose stringent limits on the possible chemical and isotopic contamination of the $\mathrm{D}_{2}$ gas. Chemical impurities (e.g. air, water) are worrisome due to large $\mu \mathrm{d} \rightarrow \mu \mathrm{Z}$ transfer rates and require concentrations of $\mathrm{N}_{2}$ of $\leq 1 \mathrm{ppb}$ and $\mathrm{O}_{2}$ of $\leq 3 \mathrm{ppb}$. Isotopic impurities are worrisome due to $p d \mu$ molecule formation and require concentrations of ordinary hydrogen of $\leq 10 \mathrm{ppm}$. To achieve such purities the $\mathrm{D}_{2}$ gas was prepared in-situ by a custom isotope separation unit and continuously cleaned of chemical impurities by a custom gas recycling unit.

The MuSun experiment is well underway at PSI. Fig. 34 shows the measured energy deposition of stopping positive and negative muons in the cryogenic TPC. For negative muons the figure indicates the occurrence of one, two and three muon-catalyzed fusions are clearly detected. Production data taking was begun in 2013 and will continue through 2015.

\section{Summary and Outlook}

We have described many contemporary projects where muons are being used as probes of fundamental atomic-, nuclear- and particle-physics properties, the 
structure of the weak interaction, and as sensitive probes of physics beyond the standard model. But, why muons, say, compared to electrons or tauons? The answer involves comparing known parameters such as mass, lifetime, and decay modes, as well as practical issues such as source yields, polarizability, and once again, lifetime. Typically, greater mass provides enhanced sensitivity to high-energy-scale physics. Access through radiative quantum loops generically scales as $\left(m_{l} / m_{\text {heavy }}\right)^{2}$, where $m_{l}$ is the lepton mass and the subscript heavy

2165 might correspond to the known $W$, or $Z$, or to an unknown new particle that couples to leptons. For example, given equally precise measurements of the lepton anomalous magnetic moments, the muon will be more sensitive than an electron by a factor of 43,00025 The tau will be 300 times better still, but it suffers from its fleeting lifetime of $0.29 \mathrm{ps}$, which impedes this and many other desired studies.

We have seen how the relatively long muon lifetime allows for the formation of muonic atoms, which, with their smaller Bohr radii, can be used to sensitively probe nucleons and nuclei. The purely leptonic, but hydrogen-like, muonium atom is a probe of QED and fundamental properties, such as the muon mass 2175 and the magnetic moment ratio to the proton. The copious production of polarized muons - which are too light to decay by the strong interaction - essentially allows for a laboratory of weak-interaction studies. Highly polarized muons, and the self-analyzing nature of their decay, are essential ingredients to many experiments.

Modern experiments involving muons are generally $n t h$-generation efforts, having been designed based on earlier pioneering work. The new experiments excel in precision and sensitivity reach, often being complemented by equally important theoretical improvements. Many experiments being built now will have the rare characteristic of "discovery" sensitivity, and an energy-scale reach complementary to or beyond that of the LHC collider program.

To summarize briefly, we first recall recent accomplishments in this field, and then list future directions with their planned sensitivities.

\subsection{Recent accomplishments}

- Muon lifetime: $\tau_{\mu^{+}}$has been measured to $2.2 \mathrm{ps,} \mathrm{(1} \mathrm{ppm)} \mathrm{by} \mathrm{the} \mathrm{Mu-}$ Lan experiment 5 . With 2nd-order electroweak corrections now computed [39], the fundamental Fermi Constant is determined to be $G_{F}=$ $1.1663787(6) \times 10^{-5} \mathrm{GeV}^{-2}(0.5 \mathrm{ppm})$.

- Decay parameters: Experiments at TRIUMF [56, 57] and PSI [51, 58] have reduced the uncertainties by up to an order of magnitude on the Michel parameters $\rho, \xi$, and $\delta$, and on the transverse and longitudinal polarization

\footnotetext{
${ }^{25}$ The electron anomalous magnetic moment has been measured [176] 2300 times more precisely compared to the muon - a spectacular achievement, which determines the fine-structure constant-but it does not close the gap.
} 
of the electron in muon decay. Global fits [62, 56] have strong implications on possible deviations of the $V$-A structure of the weak interaction. Results to date confirm standard model expectations.

- Muon anomaly: The final result of the Brookhaven $(g-2)$ experiment $[6$, when compared to steadily improved theoretical evaluations [91, 92] of the muon anomaly results in a $>3 \sigma$ deviation, possibly indicative of new physics.

- $\mathrm{cLFV}$ in $\mu \rightarrow e \gamma$ : The MEG experiment has set the world record on a test of charged lepton flavor violation finding $\operatorname{BR}(\mu \rightarrow e \gamma)<5.7 \times 10^{-13}[8]$. The limits constrain new physics involving loop processes to the $10^{3} \mathrm{TeV}$ energy scale, if one assumes maximal flavor mixing.

- Muonium HFS: The LAMPF HFS experiment measured the hyperfine splitting of two transitions involving four levels of the $1 S$ muonium atom in the high-field limit. The results [111] represent an important test of boundstate QED and established the important ratios: $\mu_{\mu} / \mu_{p}=3.183345$ 13(39) $(120 \mathrm{ppb})$, and $m_{\mu} / m_{e}=206.768277(24)$.

- Muon capture on the proton: The MuCap experiment 34 determined the $\mu^{-}+p \rightarrow n+\nu_{\mu}$ singlet capture rate by measuring the $\mu^{-}$lifetime in protium gas TPC and comparing it to $\tau_{\mu^{+}}$. Including updated radiative corrections [163, one obtains $g_{p}=8.06 \pm 0.48 \pm 0.28$, the weak-nucleon pseudoscalar coupling of the proton. The result confirms a fundamental prediction of chiral perturbation theory and concludes a fifty year effort to unambiguously determine the coupling $g_{p}$.

- Proton radius: The precise measurement [140] of two $2 S \rightarrow 2 P$ (Lamb shift) transitions in muonic hydrogen determines the proton charge radius, $r_{p}=0.84087(39) \mathrm{fm}$ [140]. The results - stunningly - are $7 \sigma$ smaller than the previous world average, which was based on $e-p$ scattering and ordinary hydrogen spectroscopy. The so-called "proton radius puzzle" remains unsolved.

\subsection{Near-term projects}

At the time of this review a number of approved projects are actively taking data or are in a construction phase. These are the ones to watch for results in the coming years; they include:

- cLFV in $\mu \rightarrow e \gamma$ : The MEG experiment upgrade of the calorimeter, tracker, and other systems will improve the energy resolution and timing required to achieve a sensitivity goal of $4 \times 10^{-14}$ in the next 4 years.

- cLFV in $\mu \rightarrow e$ conversion: COMET at J-PARC and Mu2e at Fermilab will measure the coherent conversion of a muon to an electron in the field of a nucleus at a single event sensitivity approaching $10^{-17}$, a bold, 4 orders of magnitude improvement. Superconducting solenoids are used for particle production, transport, and final spectrometer functions. 
- $\mathrm{cLFV}$ in $\mu \rightarrow$ eee: Mu3e at PSI is in a R\&D phase with an approved plan to reach a BR sensitivity of $10^{-15}$. Central to their success is the development of ultra-thin silicon tracking detectors and, for a later phase, the creation of a next-generation high-intensity muon beamline.

- Muon anomalous magnetic moment: J-PARC and Fermilab experiments 90 , 89 are being built to reach sensitivities on $\delta a_{\mu}$ of $\sim 500 \mathrm{ppb}$ and $140 \mathrm{ppb}$, respectively. They employ radically different beam delivery and storage techniques, and will consequently confront different systematic errors, an important comparison.

- Muon EDM: Both $(g-2)$ experiments can parasitically collect data sensitive to a muon EDM, with 1 to 2 orders of magnitude improvement beyond the current limit, $d_{\mu}<10^{-19} e \cdot \mathrm{cm}$. Dedicated EDM storage-ring plans, using a frozen-spin technique are promising, but are not yet approved.

- Muonium HFS: The MuSEUM experiment will explore the hyperfine structure of the muonium atom using the well-established double-resonant cavity technique for exciting spin-flip transitions. They aim for an order of magnitude increase in formed muonium atoms and two orders of magnitude increase in recorded decays compared to the most recent LAMPF experiment.

- Proton radius: CREMA will publish Lamb-shift measurements on the deuteron, helium-3, and helium-4 systems, important data to be compared to their existing hydrogen measurements. The MUSE experiment plans to measure low-energy $\mu-p$ and $e-p$ scattering at low $Q^{2}$. These data will provide an important missing clue in the enduring "proton radius puzzle."

- Muon capture on deuterium: MuSun will complete a measurement of $\mu d$ capture to $1.5 \%$ precision, which will provide a clean determination of the low-energy constant arising in the effective-field-theory description of the two nucleon weak axial current. The result is relevant for fundamental astrophysics reactions, such as $p p$ fusion and the neutrino breakup reactions in the SNO experiment.

Physicists that might identify themselves with the subfield of "Muon Physics" form a diverse group who happen to share a common and unique probe. Many of the practical issues cross traditional discipline boundaries. The word "Precision" in our title evidently describes many of the results described above, but we naturally stretch its meaning to include ultra-high sensitivity experiments as well, such as those involving rare decays. We trust that the many exciting projects described in this review will convince the reader of the prolific record of this eclectic Precision Muon Physics community and of the very bright future that lies ahead. 


\section{Acknowledgments}

The authors are grateful to our many friends and colleagues in the worldwide muon physics community who have provided us with considerable materials, figures and feedback, incorporated in this review. In particular, we have had very useful input and contributions from A. Antognini, R. Bernstein, A. de Gouvêa, M. Eides, P. Kammel, D. Kawall, G. Marshall, T. Mibe, J. Miller, T. Mori, R. Pohl, B.L. Roberts, and D. Stockinger. This effort was supported by the National Science Foundation award PHY-1205792 and the DOE Office of

Nuclear Physics award DE-FG02-97ER41020.

\section{References}

[1] C. D. Anderson, S. H. Neddermeyer, Cloud Chamber Observations of Cosmic Rays at 4300 Meters Elevation and Near Sea-Level, Phys.Rev. 50 (1936) 263-271. doi:10.1103/PhysRev.50.263

[2] J. Street, E. Stevenson, New Evidence for the Existence of a Particle of Mass Intermediate Between the Proton and Electron, Phys.Rev. 52 (1937) 1003-1004. doi:10.1103/PhysRev.52.1003.

[3] P. Kunze, Study of cosmic rays in the Wilson chamber, Z. Phys. 83 (1933) 1.

[4] P. J. Mohr, B. N. Taylor, D. B. Newell, CODATA Recommended Values of the Fundamental Physical Constants: 2010, Rev.Mod.Phys. 84 (2012) 1527-1605. arXiv:1203.5425, doi:10.1103/RevModPhys .84.1527.

[5] V. Tishchenko, et al., Detailed Report of the MuLan Measurement of the Positive Muon Lifetime and Determination of the Fermi Constant, Phys.Rev. D87 (5) (2013) 052003. arXiv:1211.0960, doi:10.1103/ PhysRevD.87.052003.

[6] G. Bennett, et al., Final Report of the Muon E821 Anomalous Magnetic Moment Measurement at BNL, Phys.Rev. D73 (2006) 072003. arXiv: hep-ex/0602035, doi:10.1103/PhysRevD.73.072003.

[7] G. Bennett, et al., An Improved Limit on the Muon Electric Dipole Moment, Phys.Rev. D80 (2009) 052008. arXiv:0811.1207, doi:10.1103/ PhysRevD.80.052008.

[8] J. Adam, et al., New constraint on the existence of the $\mu^{+} \rightarrow e^{+} \gamma$ decay, Phys.Rev.Lett. 110 (2013) 201801. arXiv:1303.0754, doi:10.1103/ PhysRevLett.110.201801.

[9] U. Bellgardt, et al., Search for the Decay $\mu^{+} \rightarrow e^{+} e^{+} e^{-}$, Nucl.Phys. B299 (1988) 1. doi:10.1016/0550-3213(88)90462-2. 
[10] W. H. Bertl, et al., A Search for muon to electron conversion in muonic gold, Eur.Phys.J. C47 (2006) 337-346. doi:10.1140/epjc/ s2006-02582-x

[11] E. Williams, G. Roberts, Evidence for transformation of mesotrons into electrons, Nature 145 (1940) 102-103.

[12] B. Rossi, D. B. Hall, Variation of the Rate of Decay of Mesotrons with Momentum, Phys.Rev. 59 (1941) 223-228. doi:10.1103/PhysRev.59. $2320 \quad 223$

[13] F. Rasetti, Disintegration of Slow Mesotrons, Phys.Rev. 60 (1941) 198204. doi:10.1103/PhysRev.60.198.

[14] R. B. Leighton, C. D. Anderson, A. J. Seriff, The energy spectrum of the decay particles and the mass and spin of the mesotron, Phys. Rev. 75 (1949) 1432-1437. doi:10.1103/PhysRev.75.1432. URL http://link.aps.org/doi/10.1103/PhysRev.75.1432

[15] L. Michel, Interaction between four half spin particles and the decay of the mu meson, Proc.Phys.Soc. A63 (1950) 514-531. doi:10.1088/ 0370-1298/63/5/311.

[16] L. Michel, A. Wightman, Muon decay, beta radioactivity, and universal fermi interaction, Phys. Rev. 93 (1954) 354-355. doi:10.1103/PhysRev. 93.354. URL http://link.aps .org/doi/10.1103/PhysRev.93.354

[17] T. Lee, C.-N. Yang, Question of Parity Conservation in Weak Interactions, Phys.Rev. 104 (1956) 254-258. doi:10.1103/PhysRev.104.254.

[18] R. Garwin, L. Lederman, M. Weinrich, Observations of the Failure of Conservation of Parity and Charge Conjugation in Meson Decays: The Magnetic Moment of the Free Muon, Phys.Rev. 105 (1957) 1415-1417. doi:10.1103/PhysRev.105.1415

${ }_{2340}^{2}$ [19] J. Friedman, V. Telegdi, Nuclear Emulsion Evidence for Parity Nonconservation in the Decay Chain $\pi^{+} \rightarrow \mu^{+} \rightarrow e^{+}$, Phys.Rev. 106 (1957) 1290-1293. doi:10.1103/PhysRev.106.1290.

[20] E. Sudarshan, R. Marshak, Chirality invariance and the universal Fermi interaction, Phys.Rev. 109 (1958) 1860-1860. doi:10.1103/PhysRev. 2345 109.1860.

[21] R. Feynman, M. Gell-Mann, Theory of Fermi interaction, Phys.Rev. 109 (1958) 193-198. doi:10.1103/PhysRev.109.193.

[22] T. Lee, M. Rosenbluth, C.-N. Yang, Interaction of Mesons With Nucleons and Light Particles, Phys.Rev. 75 (1949) 905. doi:10.1103/PhysRev.75. 2350 905 . 
[23] B. Pontecorvo, Nuclear capture of mesons and the meson decay, Phys. Rev. 72 (1947) 246-247. doi:10.1103/PhysRev.72.246.

URL http://link. aps.org/doi/10.1103/PhysRev.72.246

[24] V. W. Hughes, D. W. McColm, K. Ziock, R. Prepost, Muonium. i. muonium formation and larmor precession, Phys. Rev. A 1 (1970) 595-617. doi:10.1103/PhysRevA.1.595.

URL http://link. aps.org/doi/10.1103/PhysRevA.1.595

[25] J. A. Wheeler, Mechanism of capture of slow mesons, Phys. Rev. 71 (1947) 320-321. doi:10.1103/PhysRev.71.320. URL http://link.aps.org/doi/10.1103/PhysRev.71.320

[26] E. Fermi, E. Teller, The capture of negative mesotrons in matter, Phys. Rev. 72 (1947) 399-408. doi:10.1103/PhysRev.72.399. URL http://link.aps .org/doi/10.1103/PhysRev.72.399

[27] M. Conversi, E. Pancini, O. Piccioni, On the decay process of positive and negative mesons, Phys. Rev. 68 (1945) 232-232. doi:10.1103/PhysRev. 68.232 ,

URL http://link.aps.org/doi/10.1103/PhysRev.68.232

[28] M. Awramik, M. Czakon, A. Freitas, G. Weiglein, Precise prediction for the $\mathrm{W}$ boson mass in the standard model, Phys.Rev. D69 (2004) 053006. arXiv:hep-ph/0311148, doi:10.1103/PhysRevD.69.053006.

[29] C. Caso, et al., Review of particle physics. Particle Data Group, Eur.Phys.J. C3 (1998) 1-794. doi:10.1007/s10052-998-0104-x.

[30] K. Giovanetti, W. Dey, M. Eckhause, R. Hart, R. Hartmann, et al., Mean Life of the Positive Muon, Phys.Rev. D29 (1984) 343-348. doi:10.1103/ $2375 \quad$ PhysRevD.29.343.

[31] G. Bardin, J. Duclos, A. Magnon, J. Martino, E. Zavattini, et al., A New Measurement of the Positive Muon Lifetime, Phys.Lett. B137 (1984) 135-140. doi:10.1016/0370-2693(84)91121-3.

[32] M. Balandin, V. Grebenyuk, V. Zinov, A. Konin, A. Ponomarev, Measurement of the Lifetime of the Positive Muon, Sov.Phys.JETP 40 (1975) $811-814$.

[33] A. Barczyk, et al., Measurement of the Fermi Constant by FAST, Phys.Lett. B663 (2008) 172-180. arXiv:0707.3904, doi:10.1016/j. physletb.2008.04.006.

[34] V. Andreev, et al., Measurement of Muon Capture on the Proton to $1 \%$ Precision and Determination of the Pseudoscalar Coupling $g_{P}$,

(1) Phys.Rev.Lett. 110 (2013) 012504. arXiv:1210.6545, doi:10.1103/ PhysRevLett.110.012504 
[41] T. van Ritbergen, R. G. Stuart, Hadronic contributions to the muon life-

[44] F. Abe, et al., Observation of top quark production in $\bar{p} p$ collisions, 11 Phys.Rev.Lett. 74 (1995) 2626-2631. arXiv:hep-ex/9503002, doi:10. 1103/PhysRevLett.74.2626.

[45] S. Abachi, et al., Observation of the top quark, Phys.Rev.Lett. 74 2420 (1995) 2632-2637. arXiv:hep-ex/9503003, doi:10.1103/PhysRevLett. 74.2632 .

[46] G. Aad, et al., Observation of a new particle in the search for the Standard Model Higgs boson with the ATLAS detector at the LHC, Phys.Lett. B716 (2012) 1-29. arXiv:1207.7214 doi:10.1016/j.physletb.2012. $2425 \quad 08.020$. 
[47] S. Chatrchyan, et al., Observation of a new boson at a mass of $125 \mathrm{GeV}$ with the CMS experiment at the LHC, Phys.Lett. B716 (2012) 30-61. arXiv:1207.7235, doi:10.1016/j.physletb.2012.08.021.

[48] E. Leader, E. Predazzi, An Introduction to gauge theories and modern particle physics. Vol. 1: Electroweak interactions, the new particles and the parton model, Camb.Monogr.Part.Phys.Nucl.Phys.Cosmol. 3 (1996) 1.

[49] J. Hardy, I. Towner, Superallowed $0^{+} \rightarrow 0^{+}$nuclear decays: 2014 critical survey, with precise results for $V_{u d}$ and CKM unitarity, Phys.Rev. C91 (2) (2015) 025501. arXiv:1411.5987, doi:10.1103/PhysRevC.91.025501.

[ [50] T. Kinoshita, A. Sirlin, Muon decay with parity nonconserving interactions and radiative corrections in the two-component theory, Phys. Rev. 107 (1957) 593-599. doi:10.1103/PhysRev.107.593. URL http://link . aps .org/doi/10.1103/PhysRev.107.593

[51] N. Danneberg, W. Fetscher, K. Kohler, J. Lang, T. Schweizer, et al., Muon decay: Measurement of the transverse polarization of the decay positrons and its implications for the Fermi coupling constant and time reversal invariance, Phys.Rev.Lett. 94 (2005) 021802. doi:10.1103/PhysRevLett. 94.021802

[52] S. E. Derenzo, Measurement of the low-energy end of the $\mu^{+}$decay spectrum, Phys. Rev. 181 (1969) 1854-1866. doi:10.1103/PhysRev.181. 1854

URL http://link.aps.org/doi/10.1103/PhysRev.181.1854

[53] B. Balke, G. Gidal, A. Jodidio, K. A. Shinsky, H. M. Steiner, D. P. Stoker, ${ }_{2450} \quad$ M. Strovink, R. D. Tripp, J. Carr, B. Gobbi, C. J. Oram, Precise measurement of the asymmetry parameter $\delta$ in muon decay, Phys. Rev. D 37 (1988) 587-617. doi:10.1103/PhysRevD.37.587. URL http://link . aps . org/doi/10.1103/PhysRevD .37.587

[54] I. Beltrami, H. Burkard, R. Von Dincklage, W. Fetscher, H. Gerber, et al., Muon Decay: Measurement of the Integral Asymmetry Parameter, Phys.Lett. B194 (1987) 326-330. doi:10.1016/0370-2693(87)90552-1.

[55] A. Jodidio, B. Balke, J. Carr, G. Gidal, K. A. Shinsky, H. M. Steiner, D. P. Stoker, M. Strovink, R. D. Tripp, B. Gobbi, C. J. Oram, Search for right-handed currents in muon decay, Phys. Rev. D 34 (1986) 1967-1990. doi:10.1103/PhysRevD.34.1967 URL http://link.aps.org/doi/10.1103/PhysRevD.34.1967

[56] A. Hillairet, et al., Precision muon decay measurements and improved constraints on the weak interaction, Phys.Rev. D85 (2012) 092013. arXiv: 1112.3606, doi:10.1103/PhysRevD.85.092013. 
[67] Q. Ahmad, et al., Direct evidence for neutrino flavor transformation from neutral current interactions in the Sudbury Neutrino Observa2500 tory, Phys.Rev.Lett. 89 (2002) 011301. arXiv:nucl-ex/0204008, doi: 10.1103/PhysRevLett.89.011301

[57] J. Bueno, et al., Precise measurement of parity violation in polarized muon decay, Phys.Rev. D84 (2011) 032005. arXiv:1104.3632, doi:10.1103/ PhysRevD.84.032005.

[58] R. Prieels, O. Naviliat-Cuncic, P. Knowles, P. Van Hove, X. Morelle, et al., Measurement of the parameter $\xi^{\prime \prime}$ in polarized muon decay and implications on exotic couplings of the leptonic weak interaction, Phys.Rev. D90 (11) (2014) 112003. arXiv:1408.1472, doi:10.1103/PhysRevD.90. 112003 .

[59] W. Fetscher, H. Gerber, K. Johnson, Muon Decay: Complete Determination of the Interaction and Comparison with the Standard Model, Phys.Lett. B173 (1986) 102. doi:10.1016/0370-2693(86)91239-6.

[60] Burkard, et al., Muon Decay: Measurement of the Positron Polarization and Implications For The Spectrum Shape Parameter $\eta, V-A$ and $T$ Invariance, Phys.Lett. B150 (1985) 242-246. doi:10.1016/0370-2693(85) 90178-9.

[61] W. Eichenberger, R. Engfer, A. Van Der Schaaf, Measurement of the Parameter Eta-Bar in the Radiative Decay of the Muon as a Test of the $V-A$ Structure of the Weak Interaction, Nucl.Phys. A412 (1984) 523-533. doi:10.1016/0375-9474(84)90534-7.

[62] C. Gagliardi, R. Tribble, N. Williams, Global analysis of muon decay measurements, Phys.Rev. D72 (2005) 073002. arXiv:hep-ph/0509069 doi:10.1103/PhysRevD.72.073002

[63] Burkard, et al., Muon Decay: Measurement of the Transverse Positron Polarization and General Analysis, Phys.Lett. B160 (1985) 343. doi: 10.1016/0370-2693(85)91340-1.

[64] P. Herczeg, On Muon Decay in Left-right Symmetric Electroweak Models, Phys.Rev. D34 (1986) 3449. doi:10.1103/PhysRevD.34.3449.

[65] N. Severijns, M. Beck, O. Naviliat-Cuncic, Tests of the standard electroweak model in beta decay, Rev.Mod.Phys. 78 (2006) 991-1040. arXiv: nucl-ex/0605029, doi:10.1103/RevModPhys.78.991.

[66] Y. Fukuda, et al., Evidence for oscillation of atmospheric neutrinos, Phys.Rev.Lett. 81 (1998) 1562-1567. arXiv:hep-ex/9807003, doi:10. 1103/PhysRevLett.81.1562. 
[68] K. Eguchi, et al., First results from KamLAND: Evidence for reactor anti-neutrino disappearance, Phys.Rev.Lett. 90 (2003) 021802. arXiv: hep-ex/0212021, doi:10.1103/PhysRevLett.90.021802.

2505

[69] Y. Kuno, Y. Okada, Muon decay and physics beyond the standard model, Rev.Mod.Phys. 73 (2001) 151-202. arXiv:hep-ph/9909265, doi : 10.1103/RevModPhys.73.151.

[70] W. J. Marciano, T. Mori, J. M. Roney, Charged Lepton Flavor Violation Experiments, Ann.Rev.Nucl.Part.Sci. 58 (2008) 315-341. doi:10.1146/ annurev.nucl.58.110707.171126

[71] A. de Gouvea, P. Vogel, Lepton Flavor and Number Conservation, and Physics Beyond the Standard Model, Prog.Part.Nucl.Phys. 71 (2013) 7592. arXiv:1303.4097, doi:10.1016/j.ppnp.2013.03.006.

[72] R. H. Bernstein, P. S. Cooper, Charged Lepton Flavor Violation: An Experimenter's Guide, Phys.Rept. 532 (2013) 27-64. arXiv:1307.5787, doi:10.1016/j.physrep.2013.07.002.

[73] T. Mori, W. Ootani, Flavour violating muon decays, Prog.Part.Nucl.Phys. 79 (2014) 57-94. doi:10.1016/j.ppnp.2014.09.001.

[74] T. Abe, et al., Belle II Technical Design Report arXiv:1011.0352.

2520

[75] R. Aaij, et al., Search for the lepton flavour violating decay $\mathrm{t}^{-}$? $^{-}+{ }^{-}$, JHEP 1502 (2015) 121. arXiv:1409.8548, doi:10.1007/JHEP02(2015) 121 .

[76] L. Willmann, P. Schmidt, H. Wirtz, R. Abela, V. Baranov, et al., New bounds from searching for muonium to anti-muonium conversion,

2525 - Phys.Rev.Lett. 82 (1999) 49-52. arXiv:hep-ex/9807011, doi:10.1103/ PhysRevLett.82.49.

q [77] B. Liu, Muonium-antimuonium oscillations in an extended minimal supersymmetric standard model with right-handed neutrinos, Phys. Rev. D 79 (2009) 015001. doi:10.1103/PhysRevD .79.015001. URL http://link.aps.org/doi/10.1103/PhysRevD.79.015001

[78] J. Adam, X. Bai, A. Baldini, E. Baracchini, C. Bemporad, et al., The MEG detector for $\mu^{+} \rightarrow e^{+} \gamma$ decay search, Eur.Phys.J. C73 (4) (2013) 2365. arXiv:1303.2348, doi:10.1140/epjc/s10052-013-2365-2.

[79] A. Baldini, F. Cei, C. Cerri, S. Dussoni, L. Galli, et al., MEG Upgrade Proposa arXiv: 1301.7225 .

[80] N. Berger, The Mu3e Experiment, Nucl.Phys.Proc.Suppl. 248-250 (2014) 35-40. doi:10.1016/j.nuclphysbps.2014.02.007. 
[81] H. Natori, DeeMe experiment - An experimental search for a mu-e conversion reaction at J-PARC MLF, Nucl.Phys.Proc.Suppl. 248-250 (2014) 52-57. doi:10.1016/j.nuclphysbps.2014.02.010.

[82] L. Bartoszek, et al., Mu2e Technical Design Report: FERMILAB-TM2594 .

[83] Y. Cui, et al., Conceptual design report for experimental search for lepton flavor violating $\mu^{-} \rightarrow e^{-}$conversion at sensitivity of $10^{-16}$ with a slow-extracted bunched proton beam (COMET), COMET Collaboration: KEK-2009-10 (2009).

[84] R. Dzhilkibaev, V. Lobashev, On the Search for $\mu \rightarrow e$ Conversion on Nuclei. (In Russian), Sov.J.Nucl.Phys. 49 (1989) 384-385.

[85] A. Czarnecki, X. Garcia i Tormo, W. J. Marciano, Muon decay in orbit: spectrum of high-energy electrons, Phys.Rev. D84 (2011) 013006. arXiv: 1106.4756, doi:10.1103/PhysRevD.84.013006.

[86] F. Jegerlehner, The anomalous magnetic moment of the muon, Springer Tracts Mod.Phys. 226 (2008) 1-426. doi:10.1007/978-3-540-72634-0.

[87] F. Jegerlehner, A. Nyffeler, The Muon g-2, Phys.Rept. 477 (2009) 1-110. arXiv:0902.3360, doi:10.1016/j.physrep.2009.04.003

[88] F. Farley, Y. Semertzidis, The 47 years of muon g-2, Prog.Part.Nucl.Phys. 52 (2004) 1-83. doi:10.1016/j.ppnp.2003.09.004.

[89] J. Grange, et al., Muon (g-2) Technical Design Report: FERMILAB-FN0992-E.

[90] H. Iinuma, New approach to the muon g-2 and EDM experiment at J-

1 PARC, J.Phys.Conf.Ser. 295 (2011) 012032. doi:10.1088/1742-6596/ 295/1/012032.

[91] M. Davier, A. Hoecker, B. Malaescu, Z. Zhang, Reevaluation of the Hadronic Contributions to the Muon g-2 and to alpha(MZ), Eur.Phys.J. C71 (2011) 1515. arXiv:1010.4180, doi:10.1140/epjc/ s10052-012-1874-8,10.1140/epjc/s10052-010-1515-z.

[92] K. Hagiwara, R. Liao, A. D. Martin, D. Nomura, T. Teubner, $(g-2)_{\mu}$ and $\alpha\left(M_{Z}^{2}\right)$ re-evaluated using new precise data, J.Phys. G38 (2011) 085003. arXiv:1105.3149, doi:10.1088/0954-3899/38/8/085003

[93] T. Blum, A. Denig, I. Logashenko, E. de Rafael, B. Lee Roberts, et al., White Paper: The Muon (g-2) Theory Value: Present and Future: arXiv:1311.2198 (2013).

[94] Benayoun, et al., Workshop on the Hadronic contributions to the muon anomalous magnetic moment: arXiv:1407.4021 (2014). 
[107] D. Hertzog, W. Morse, The Brookhaven muon anomalous magnetic mo1 ment experiment, Ann.Rev.Nucl.Part.Sci. 54 (2004) 141-174. doi:10. 1146/annurev.nucl.53.041002.110618.

[108] J. P. Miller, E. de Rafael, B. L. Roberts, Muon (g-2): Experiment and 1 theory, Rept.Prog.Phys. 70 (2007) 795. arXiv:hep-ph/0703049, doi: 10.1088/0034-4885/70/5/R03. 
[109] V. Bargmann, L. Michel, V. Telegdi, Precession of the polarization of particles moving in a homogeneous electromagnetic field, Phys.Rev.Lett. 2 (1959) 435. doi:10.1103/PhysRevLett.2.435.

[110] G. Bennett, et al., Statistical equations and methods applied to the precision muon (g-2) experiment at BNL, Nucl.Instrum.Meth. A579 (2007) 1096-1116. doi:10.1016/j.nima.2007.06.023.

[111] W. Liu, M. G. Boshier, S. Dhawan, O. van Dyck, P. Egan, et al., High precision measurements of the ground state hyperfine structure interval of muonium and of the muon magnetic moment, Phys.Rev.Lett. 82 (1999) 711-714. doi:10.1103/PhysRevLett.82.711.

[112] G. Danby, L. Addessi, Z. Armoza, J. Benante, H. Brown, et al., The Brookhaven muon storage ring magnet, Nucl.Instrum.Meth. A457 (2001) 151-174. doi:10.1016/S0168-9002(00)00704-X.

[113] A. Fienberg, L. Alonzi, A. Anastasi, R. Bjorkquist, D. Cauz, et al., Studies of an array of PbF2 Cherenkov crystals with large-area SiPM readout, Nucl.Instrum.Meth. A783 (2015) 12-21. arXiv:1412.5525, doi:10.1016/j.nima.2015.02.028.

[114] G. A. Beer, Y. Fujiwara, S. Hirota, K. Ishida, M. Iwasaki, et al., Enhancement of muonium emission rate from silica aerogel with a laser

n ablated surface, PTEP 2014 (2014) 091C01. arXiv:1407.8248, doi: 10.1093/ptep/ptu116.

[115] V. Hughes, Muonium, Ann.Rev.Nucl.Part.Sci. 16 (1966) 445-470. doi: 10.1146/annurev.ns.16.120166.002305

[116] K. S. Khaw, Towards next generation fundamental precision measurements with muons, Ph.D. Thesis: DISS. ETH No. 22549 (2015).

[117] T. Mibe, The silicon detector of the muon g-2 experiment at JPARC, PoS Vertex2011 (2011) 044.

[118] M. Dine, A. Kusenko, The Origin of the matter - antimatter asymmetry, Rev.Mod.Phys. 76 (2003) 1. arXiv:hep-ph/0303065, doi:10.1103/ RevModPhys.76.1.

[119] D. E. Morrissey, M. J. Ramsey-Musolf, Electroweak baryogenesis, New J.Phys. 14 (2012) 125003. arXiv:1206.2942, doi:10.1088/1367-2630/

\section{$2645 \quad 14 / 12 / 125003$}

[120] T. Chupp, M. Ramsey-Musolf, Electric Dipole Moments: A Global Anal口 ysis, Phys.Rev. C91 (3) (2015) 035502. arXiv:1407.1064, doi:10.1103/ PhysRevC.91.035502.

[121] K. Babu, S. M. Barr, I. Dorsner, The Scaling of lepton dipole moments with lepton mass, Phys.Rev. D64 (2001) 053009. arXiv: hep-ph/0012303, doi:10.1103/PhysRevD.64.053009. 
[122] G. Hiller, K. Huitu, T. Ruppell, J. Laamanen, A Large Muon Electric Dipole Moment from Flavor?, Phys.Rev. D82 (2010) 093015. arXiv: 1008. 5091, doi:10.1103/PhysRevD .82.093015

[127] C. Baker, D. Doyle, P. Geltenbort, K. Green, M. van der Grinten, et al., An Improved experimental limit on the electric dipole moment of the neutron, Phys.Rev.Lett. 97 (2006)
10.1103/PhysRevLett.97.131801

[128] F. J. M. Farley, K. Jungmann, J. P. Miller, W. M. Morse, Y. F. Orlov, B. L. Roberts, Y. K. Semertzidis, A. Silenko, E. J. Stephenson, New method of measuring electric dipole moments in storage rings, Phys. Rev. Lett. 93 URL http://link.aps.org/doi/10.1103/PhysRevLett.93.052001

[129] A. Adelmann, K. Kirch, C. Onderwater, T. Schietinger, Compact storage ring to search for the muon electric dipole moment, J.Phys. G37 (2010) 085001. doi:10.1088/0954-3899/37/8/085001.

[130] B. D. Claude Cohen-Tannoudji, F. Laloe, Quantum Mechanics, Volume 2, Hermann, Paris,France, 1977.

[131] R. P. Feynman, R. B. Leighton, M. Sands, The Feynman Lectures on Physics, Volume III.

[132] V. Hughes, D. McColm, K. Ziock, R. Prepost, Formation of Muonium and Observation of its Larmor Precession, Phys.Rev.Lett. 5 (1960) 63-65. doi:10.1103/PhysRevLett.5.63.

[133] R. De Voe, P. Mcintyre, A. Magnon, D. Stowell, R. Swanson, et al., Measurement of the muonium hfs splitting and of the muon moment by *double resonance*, and a new value of alpha, Phys.Rev.Lett. 25 (1970) 17791783. doi:10.1103/PhysRevLett.25.1779. 
[134] V. Meyer, S. Bagaev, P. Baird, P. Bakule, M. Boshier, et al., Measurement of the 1s - 2s energy interval in muonium, Phys.Rev.Lett. 84 (2000) 1136. arXiv:hep-ex/9907013, doi:10.1103/PhysRevLett.84.1136.

[143] R. Milner, et al., The OLYMPUS Experiment, Nucl.Instrum.Meth. A741 (2014) 1-17. arXiv:1312.1730, doi:10.1016/j.nima.2013.12.035.

[144] J. Bernauer, et al., High-precision determination of the electric and magnetic form factors of the proton, Phys.Rev.Lett. 105 (2010) 242001.

[145] R. Gilman, et al., Studying the Proton "Radius" Puzzle with $\mu p$ Elastic ScatteringarXiv: 1303.2160 .

[146] J. A. Wheeler, Some Consequences of the Electromagnetic Interaction

a between mu-Mesons and Nuclei, Rev.Mod.Phys. 21 (1949) 133-143. doi: 2725

[147] R. H. Hildebrand, Observation of muon capture in liquid hydrogen, Phys. Rev. Lett. 8 (1962) 34-37. doi:10.1103/PhysRevLett.8.34. URL http://link.aps.org/doi/10.1103/PhysRevLett.8.34 
[148] S. Weinberg, Charge symmetry of weak interactions, Phys.Rev. 112 (1958) 1375-1379. doi:10.1103/PhysRev.112.1375.

[149] J. Hardy, I. Towner, Superallowed $0^{+} \rightarrow 0^{+}$nuclear beta decays: A New survey with precision tests of the conserved vector current hypothesis and the standard model, Phys.Rev. C79 (2009) 055502. arXiv:0812.1202, doi:10.1103/PhysRevC.79.055502.

2735

[150] K. Minamisono, T. Nagatomo, K. Matsuta, C. Levy, Y. Tagishi, et al., Low-energy test of second-class current in beta decays of spin-aligned F-20

n and Na-20, Phys.Rev. C84 (2011) 055501. doi:10.1103/PhysRevC.84. 055501 .

[151] M. Gell-Mann, Test of the Nature of the Vector Interaction in beta Decay, Phys.Rev. 111 (1958) 362-365. doi:10.1103/PhysRev.111.362.

[152] M. Gell-Mann, M. Levy, The axial vector current in beta decay, Nuovo Cim. 16 (1960) 705. doi:10.1007/BF02859738.

[153] V. Bernard, N. Kaiser, U. G. Meissner, QCD accurately predicts the induced pseudoscalar coupling constant, Phys.Rev. D50 (1994) 6899-6901. arXiv:hep-ph/9403351, doi:10.1103/PhysRevD.50.6899

[154] T. Gorringe, H. W. Fearing, Induced pseudoscalar coupling of the pro-

10 ton weak interaction, Rev.Mod.Phys. 76 (2004) 31-91. arXiv:nucl-th/ 0206039, doi:10.1103/RevModPhys.76.31

[155] S. Pastore, F. Myhrer, K. Kubodera, An update of muon capture on hydrogen, Int.J.Mod.Phys. E23 (2014) 1430010. arXiv:1405.1358, doi: 10.1142/S0218301314300100.

[156] A. Badertscher, M. Daum, R. Frosch, P. Goudsmit, W. Hajdas, et al., Experimental determination of the kinetic energy distribution of pi- $\mathrm{p}$ atoms in liquid hydrogen, Phys.Lett. B392 (1997) 278-282. doi:10.1016/ $2755 \quad$ S0370-2693(96)01545-6.

[157] V. Andreev, et al., Measurement of the Formation Rate of Muonic Hydrogen Molecules arXiv: 1502.00913.

[158] D. Bakalov, M. Faifman, L. Ponomarev, S. Vinitsky, $\mu$ Capture and Ortho - Para Transitions in Mesic Molecule pp $\mu$, Nucl.Phys. A384 (1982) 302. doi:10.1016/0375-9474(82)90337-2.

[159] G. Bardin, J. Duclos, A. Magnon, J. Martino, A. Richter, et al., Measurement of the Ortho - Para Transition Rate in the $p \mu p$ Molecule and Deduction of the Pseudoscalar Coupling Constant, Phys.Lett. B104 (1981) 320. doi:10.1016/0370-2693(81)90134-9. 
[162] D. Wright, S. Ahmad, D. Armstrong, G. Azuelos, W. Bertl, et al., Measurement of the induced pseudoscalar coupling using radiative muon capture on hydrogen, Phys.Rev. C57 (1998) 373-390. doi:10.1103/ PhysRevC.57.373.

[163] A. Czarnecki, W. J. Marciano, A. Sirlin, Electroweak radiative corrections to muon capture, Phys.Rev.Lett. 99 (2007) 032003. arXiv:0704.3968, doi:10.1103/PhysRevLett.99.032003.

[164] J. Beringer, et al., Review of Particle Physics (RPP), Phys.Rev. D86 (2012) 010001. doi:10.1103/PhysRevD.86.010001.

[165] N. Tatara, Y. Kohyama, K. Kubodera, Weak interaction processes on deuterium: Muon capture and neutrino reactions, Phys.Rev. C42 (1990) 1694-1717. doi:10.1103/PhysRevC.42.1694.

[166] M. Doi, T. Sato, H. Ohtsubo, M. Morita, Muon capture in hyperfine n states of muonic deuterium, Prog.Theor.Phys. 86 (1991) 13-16. doi: 10.1143/PTP.86.13.

[167] J. Adam, E. Truhlik, S. Ciechanowicz, K. Schmitt, Muon capture in deuterium and the meson exchange current effect, Nucl.Phys. A507 (1990) 675-697. doi:10.1016/0375-9474(90)90175-L.

[168] L. Marcucci, A. Kievsky, S. Rosati, R. Schiavilla, M. Viviani, Chiral effective field theory predictions for muon capture on deuteron and ${ }^{3} \mathrm{He}$, Phys.Rev.Lett. 108 (2012) 052502. arXiv:1109.5563, doi:10.1103/ PhysRevLett.108.052502.

[169] J. Adam, et al., Calculation of Doublet Capture Rate for Muon Capture in Deuterium within Chiral Effective Field Theory, Phys.Lett. B709 (2012)

n 93-100. arXiv:1110.3183, doi:10.1016/j.physletb.2012.01.01065, $10.1016 / \mathrm{j}$.physletb.2012.01.065

[170] P. Kammel, et al., First Observation of Muonic Hyperfine Effects in Pure 2800 Deuterium, Phys.Rev. A28 (1983) 2611-2622. doi:10.1103/PhysRevA. 28.2611. 
[171] W. Breunlich, P. Kammel, J. Cohen, M. Leon, Muon-catalyzed fusion, Ann.Rev.Nucl.Part.Sci. 39 (1989) 311-356. doi:10.1146/annurev.ns. 39.120189 .001523

2805 [172] F. Frank, Hypothetical Alternative Energy Sources for the Second Meson Events, Nature 160 (1947) 525-575. doi:10.1038/160525a0.

[173] L. Alvarez, H. Bradner, J. Crawford, F.S., P. Falk-Vairant, M. Good, et al., Catalysis of nuclear reactions by mu mesons, Phys. Rev. 105 (1957) 1127-1128. doi:10.1103/PhysRev.105.1127.

2810 [174] G. G. Bardin, J. Duclos, J. Martino, A. Bertin, M. Capponi, M. Piccinini, A. Vitale, A measurement of the muon capture rate in liquid deuterium

口 by the lifetime technique, Nucl. Phys. 453 (1986) 591-604. doi:10.1016/ 0375-9474(86) 90253-8.

[175] M. Cargnelli, et al., Measurement of the muon capture rate in gaseous deuterium, Nuclear Weak Process and Nuclear Stucture, Yamada Conference XXIII, Ed. by M. Morita, H. Ejiri, H. Ohtsubo, and T. Sato, (World Scientific Pub.) 453 (1989) 115.

[176] D. Hanneke, S. Fogwell, G. Gabrielse, New Measurement of the Electron Magnetic Moment and the Fine Structure Constant, Phys.Rev.Lett.

2820 a 100 (2008) 120801. arXiv:0801.1134, doi:10.1103/PhysRevLett.100. 120801 . 